\title{
A PATIENT-SPECIFIC, DYNAMIC, MULTIOBJECTIVE MODEL FOR HEPATOCELLULAR CARCINOMA TREATMENTS
}

\author{
A Thesis \\ Presented to \\ the faculty of the School of Engineering and Applied Science \\ University of Virginia \\ In Partial Fulfillment \\ of the requirements for the Degree \\ Master of Science in Systems Engineering
}

by

Evan Paul Rust

May 2013 


\section{APPROVAL SHEET}

The thesis is submitted in partial fulfillment of the requirements for the degree of Master of Science in Systems Engineering

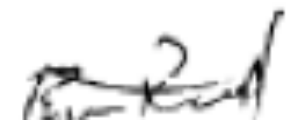

Evan Paul Rust (author)

This thesis has been read and approved by the examining Committee:

Dr. Yacov Haimes (advisor)

Dr. Barry Horowitz (chair)

Dr. Carl Berg

Dr. Patrick Northup

Accepted for the School of Engineering and Applied Science:

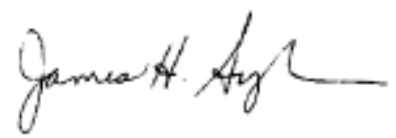

Dean, School of Engineering and Applied Science 


\section{ABSTRACT}

This study addresses a systems-based approach for healthcare delivery to patients with hepatocellular carcinoma (HCC), the most common type of liver cancer, who are awaiting orthotopic liver transplantation (OLT). Often waiting as long as a year for OLT, these patients undergo many intermediary treatments, the sequence of which uniquely changes over time for each patient. These combinations of therapies are too many in number for a physician to evaluate accurately in his mind, as he must administer the most effective therapy at each checkup. To overcome these limitations, this study describes a dynamic multiobjective decision tree (D-MODT) designed by the author to provide medical decisionmakers with Pareto-optimal treatment strategies for patients suffering from HCC. A multistage decision tree framework is developed, allowing the model to be customized for each patient in terms of state of health and geographic location by updating via Bayes' Theorem a patient's probabilities of survival, progression, etc. as every piece of new information arrives to the physician. This level of refinement has not achieved, to the author's knowledge, in any study employing the traditional Markovian approach, where only transitions from the most recent state of health are considered. The model's framework and mathematics are described herein, along with a case study that details how a 1-month case of the model would be solved and analyzed. The model is populated with data from UNOS and the relevant literature, although data collection and better population of the model are large future directions for this research. Although this methodological approach is herein applied to HCC treatment, its success should encourage its application in other areas of medicine where complex sequential decisionmaking confounds physicians in disease management scenarios. 


\section{TABLE OF CONTENTS}

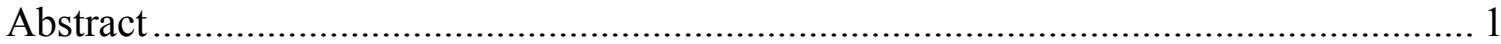

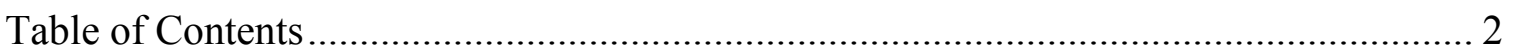

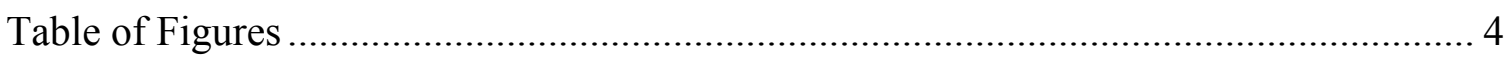

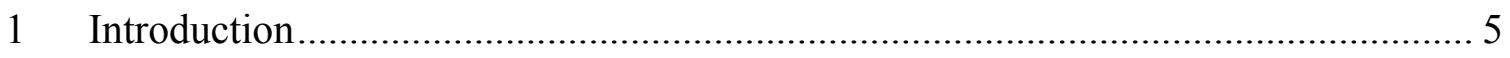

1.1 Problem Statement .................................................................................... 8

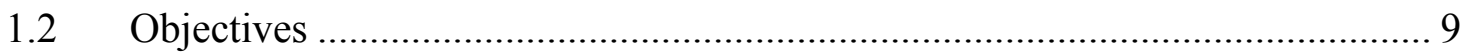

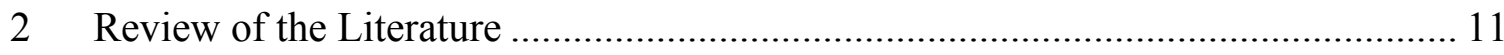

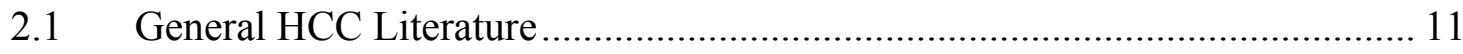

2.2 Clinical Literature Review ..................................................................... 14

2.3 Markov Models: Overview and Clinical Studies.......................................... 18

2.3.1 Limitations of Markov Models ............................................................ 21

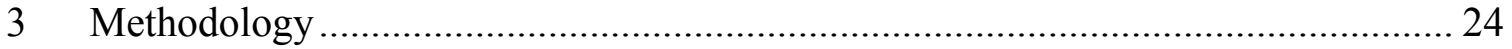

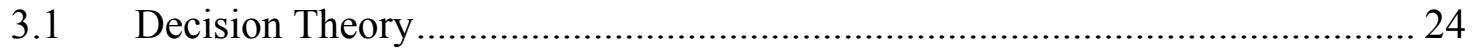

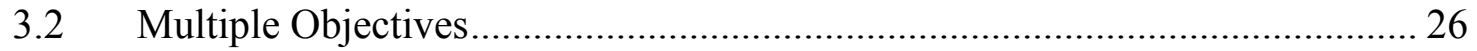

3.3 Multi-Objective Decision Trees (MODTs) ................................................... 28

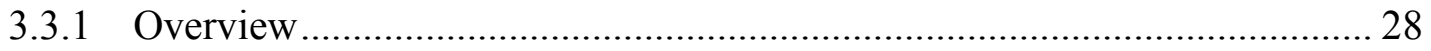

3.3.2 Solution Procedure ................................................................................. 30

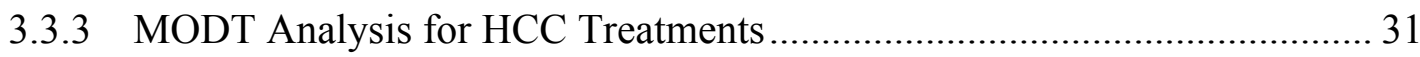

4 Dynamic Multiobjective Decision Tree for HCC .............................................. 34

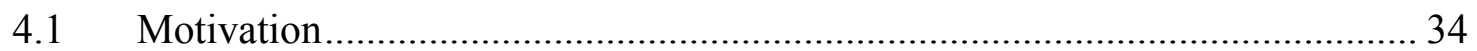

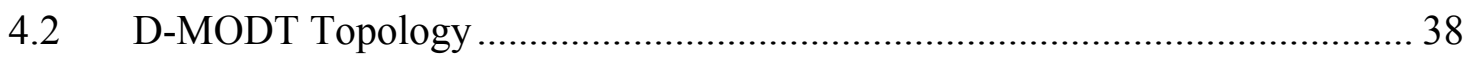

4.2.1 State Variable and MELD Discussion ........................................................ 41

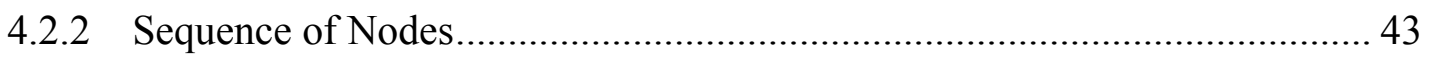

4.2.3 Complete D-MODT Structure ........................................................... 47

4.2.4 Mathematical Solution Procedure ........................................................... 48

4.3 Advantages of D-MODT Methodology ..................................................... 51

5 D-MODT for HCC: An Explanative Case Study …......................................... 55

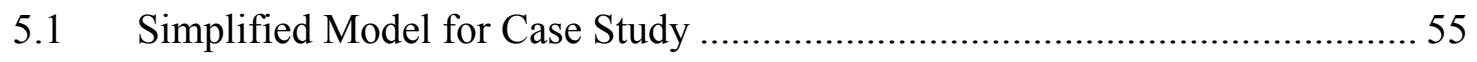

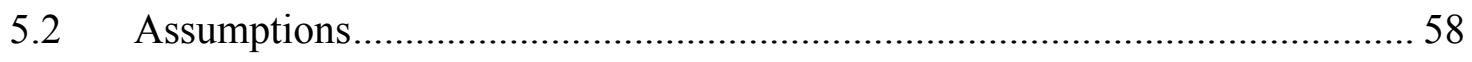

5.3 Review of the Database and Model Population........................................... 59

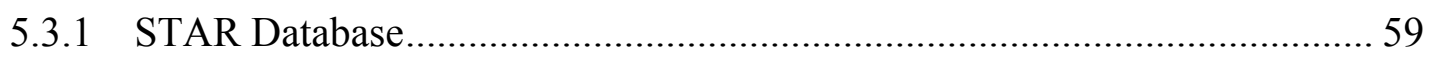

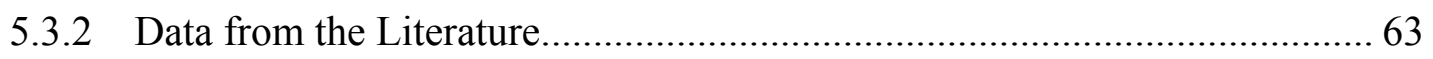




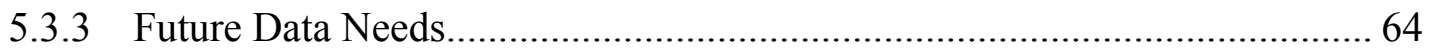

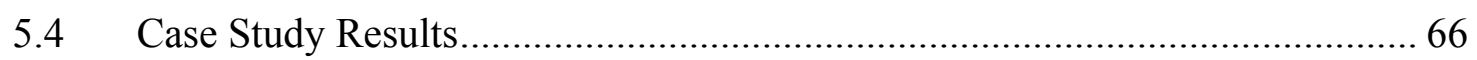

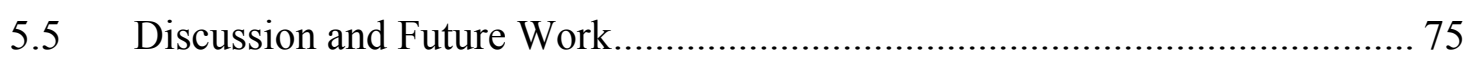

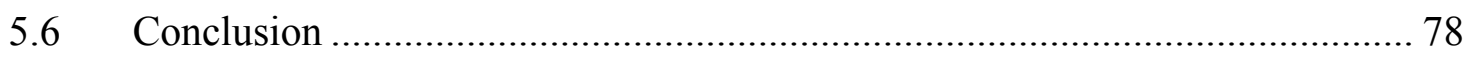

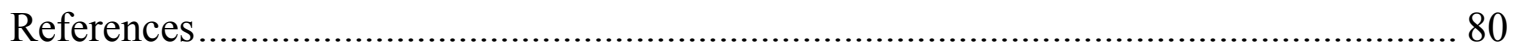

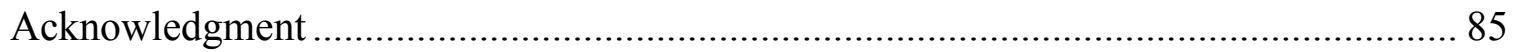

Appendix A: Median Wait Times by Meld and Region ................................................ 86

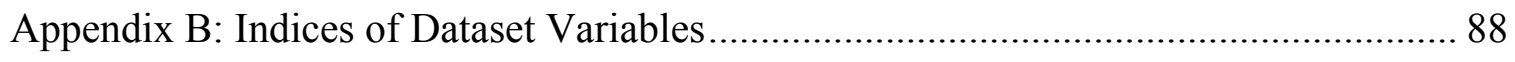

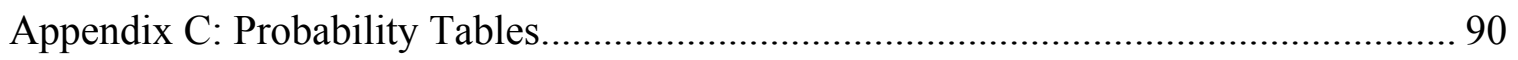

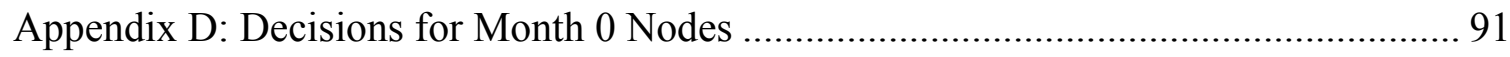

Appendix E: Graphs of Strategies (All and Optimal) ………………....................... 113 


\section{TABLE OF FIGURES}

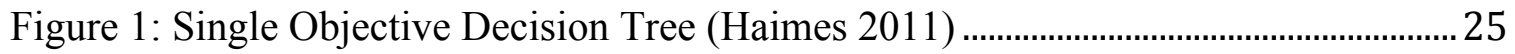

Figure 2: Sample Pareto-Optimal Frontier............................................................................ 28

Figure 3: Structure of Multi-Objective Decision Trees (Haimes, et al. 1990) ..................... 29

Figure 4: Simplified MODT for HCC (Bleistein, 2011) ........................................................... 31

Figure 5: Simplified Pre-Transplant D-MODT ……….............................................................. 39

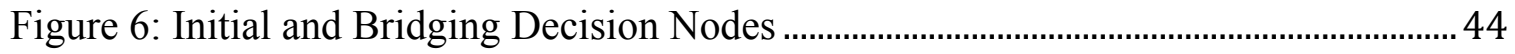

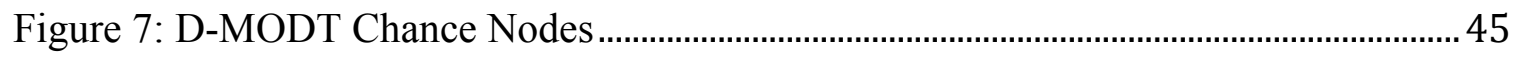

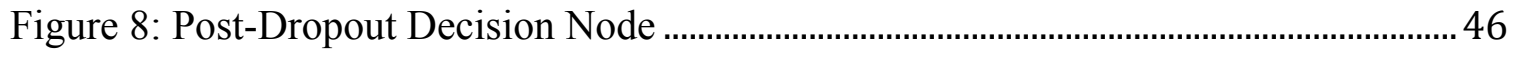

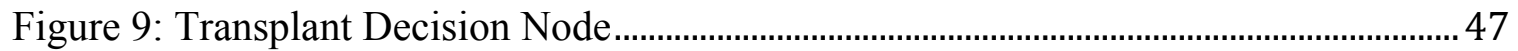

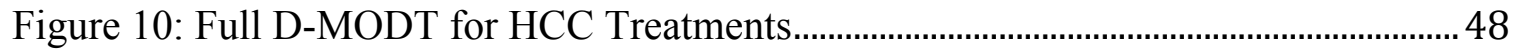

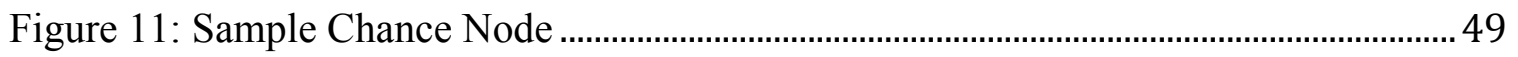

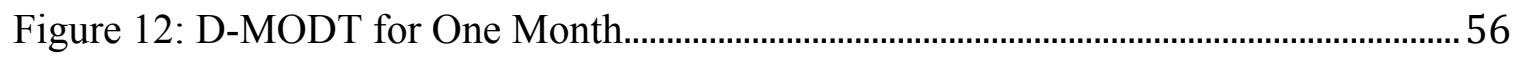

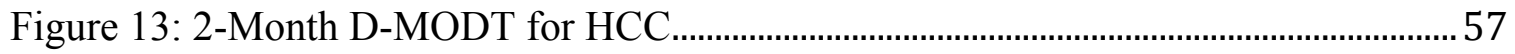




\section{INTRODUCTION}

Hepatocellular carcinoma is the most common form of liver cancer, the fifth most-common type of cancer, and the third most-common cause of cancer-related death worldwide (Llovet, Fuster, \& Bruix, 2004). In the early 2000s, there were between 8,500-11,500 new U.S. cases of HCC annually, and these cases carried 1 and 3-year survival rates of $36 \%$ and $17 \%$, respectively (El-Serag, 2004). This already-major problem will only continue to rise in incidence in the next decade due to the future prevalence of Hepatitis C (Davis, Alter, El-Serag, Poynard, \& Jennings, 2010). When possible, liver transplantation or resection are the firstline treatment options for HCC patients, as "optimal candidates" have a 5-year survival rate of over $70 \%$ posttransplant (Llovet, Fuster, \& Bruix, 2004). Resection, however, is only a viable option in $5 \%$ of cases in the West. Optimal candidates for transplantation are termed as such by their meeting the so-called "Milan criteria," which states that they must have either one tumor not exceeding $5 \mathrm{~cm}$ or 3 tumors not exceeding $3 \mathrm{~cm}$ (Llovet, Fuster, \& Bruix, 2004). Clearly, liver transplantation has marked effects on survival rates, as this 5-year survival rate of over $70 \%$ is much preferred to the 3 -year untreated survival rate of only $28 \%$ (Llovet, Burroughs, \& Bruix, 2003). Yet, the disequilibrium between the demand for transplantable organs and the supply of available livers has resulted in the waiting period for transplantation to often exceed 12 months (Llovet, et al., 2002). These lengthy wait times also increase the number of patients whose declining health causes them to be removed from transplant consideration ( $23 \%$ will drop out within six months), thus further hurting their chances for survival (Llovet, et al., 2002). 
Given that liver transplantation is the primary treatment option for HCC patients, there has been much time and energy devoted to devising a system that appropriates organs to patients in need. Since 2002, the Model for End-Stage Liver Disease (MELD) system has been utilized to allocate deceased donor livers in the United States (Kamath \& Kim, 2007). MELD assigns a score to patients awaiting liver transplantation based on various factors that determine the risk of patient death from liver disease within 90 days of their arrival on the wait list for transplantation. This model was not designed exclusively for HCC, as it determines the risk of death from intrinsic liver disease rather than from liver cancer. Subsequently, some argued that MELD assigned low scores to HCC patients (who had lesser risk of dying from liver disease) who otherwise had a high risk of death from liver cancer; that is, MELD may have underestimated the risk of death for HCC patients (Volk, 2010). As a result, HCC patients are assigned a priority score of 22 and given an additional 3 points every 3 months until they die, they are transplanted, or their tumor becomes too large.

Lengthy wait times for transplantation have caused physicians to administer "bridging" therapies, with the goal of maintaining a patient's health so that he does not dropout from consideration while he awaits transplantation. The set of these therapies includes trans-arterial chemoembolization (TACE), radiofrequency ablation (RFA), percutaneous ethanol injection (PEI), and internal radiation via Yttrium ${ }^{90}$ microspheres (Llovet, et al., 2002; Naugler \& Sonnenberg, 2010; Salem, et al., 2009). In addition, many physicians treat with sorafenib after a patient has dropped out from transplant consideration (Llovet, et al., 2008). These therapies have been extensively studied and compared in the aforementioned papers. In the literature, most treatment studies were 
simulated in three-month cycles, which is akin to UVA's practice of a patient being scanned (via CT/MRI) one month after the introduction of any therapy, and every three months as he remains on the same therapy (Naugler \& Sonnenberg, 2010).

This combination of multiple competing therapeutic options that are available for administration to the patient every one or three months throughout his (often) lengthy treatment quickly spans into a large combinatorial network of decisions. With the assumption of three available treatments at every checkup, the physician is presented with a network of $3^{4}=81$ different treatment sequences by only the fourth checkup. Miller, in his seminal paper, observed that humans are simply unable to make sound decisions among a surprisingly small number (capped at 9) number of alternatives (Miller, 1956). This hypothesis that decision-makers cannot process large numbers of alternatives on their own necessitates the development of technology to come to their aid. This thesis describes one such tool in the context of HCC treatment.

The primary objective of this study is to develop a tool to aid medical decisionmakers in their management of HCC in ways that existing models cannotnamely, the Markovian limitations of accommodating a heterogenetic patient cohort within the model, and pursuing greater levels of patient specificity. For an individual patient, our model will output the Pareto-optimal (to be defined in a later section) set of treatments for the number of months that corresponds to the median wait time for his region. Future research should develop a solution algorithm in Objective-C and a subsequent iOS application so that a physician may input his patient's location (i.e., UNOS region) and state of health (i.e., MELD score at listing) and quickly view a list of 
optimal strategies (in terms of lifetime provided and cost incurred) and their respective quantitative tradeoffs.

On a higher level, this methodology will allow researchers to simulate test cases in the theoretical rather than the physical realm. The ability to simulate therapeutic schedules that would be unfeasible or unethical to administer to actual patients will be of great value to researchers seeking to develop baseline statistics for HCC and other diseases.

\subsection{Problem Statement}

HCC treatment is not "one-size-fits-all" in any sense. Each patient is characterized by his unique state of health both at the beginning of and at every point throughout his treatment, and different therapies are available to him at different times given these continuously updated states of health. Depending on his UNOS region and MELD score, a patient may receive a transplant rather briefly after listing, or wait a year until a donor liver is available. Because of the uniqueness of pre-transplant treatment across the U.S., there is no established sequence of therapies month-to-month.

The disequilibrium between the supply of and demand for donor livers further confounds the problem of HCC treatment, as the limited availability of transplantable organs emphasizes that they are allocated to the patients who will most benefit (in terms of lifetime provided) from them. The Organ Procurement and Transplantation Network (OPTN) keeps an updated online record of the U.S. waiting list. According to their national data, there are 15,720 candidates on the waitlist for liver transplantation as of March 4, 2013. Yet, in 2012, there were only 6,633 deceased donors (and 246 living 
donors) added to the waitlist as compared to 11,609 new candidates. The majority (72.61\%) of these patients were at least the age of 50 upon their listing. Thus, given the severity of the problem at hand and the necessity of a patient-specific approach, the problem examined in this thesis is how best to model HCC treatment's sequential decision-making. This is not a clinical cohort study, a retrospective chart review per se, or a study designed to inform physicians of the most efficacious bridging therapy; rather, this thesis seeks to lay the foundations for a dynamic modeling approach that, once sufficient data are available, can be populated and customized for any combination of patient and available therapies. It should be noted that the therapies, MELD scoring buckets, and patients used later in this paper's hypothetical case study are meant to serve as an only as examples and that the model can theoretically be refined to any combination thereof and be solved via the same mathematical technique.

\subsection{Objectives}

This thesis seeks to extend the work started by Bleistein (2011) in his effort to: Develop a system-based methodology that would assist clinicians and the medical community involved in liver transplantation to evaluate the trade-offs among the multiple options available for the treatment of HCC patients, including the allocation of living and deceased livers to patients of varied health conditions. Bleistein built a static model to analyze the tradeoffs between pre-transplant bridging therapies. His work will be further detailed in the review of the literature.

The work contained herein seeks to complete the next modeling iteration, based off Bleistein's prior modeling efforts. Specifically, this work develops a methodology for 
dynamic multiobjective decision trees (D-MODT), from which comes a model for HCC centered on the importance of the patient's state of health. While at this present moment sufficient data is not available to populate a full D-MODT for a patient with a lengthy wait time or an unorthodox (yet theoretically feasible) combination of therapies over time, the purpose of this work is rather to demonstrate the advantages of the D-MODT methodology vis-à-vis other methodologies (including but not limited to Markov models), the importance of the state variable, and the benefit of incorporating patient specificity.

In sum, this work seeks to provide researchers with an improved methodology with which to later, when sufficient data are available, populate large-scale models and accurately evaluate the tradeoffs among the vast combinations of therapeutic sequences possible during HCC treatment. More future directions for this work will be discussed in a later section. 


\section{REVIEW OF THE LITERATURE}

Throughout the years, HCC has been the subject of many studies. In this section, several papers are summarized in order to present the reader with a clearer view of the work that has already been done, and also to expose to him the space that the model presented in this paper attempts to fill. These papers served as both motivation for this current work and also provided the model with several data points that could not be determined from the OPTN database alone.

\subsection{General HCC Literature}

The following papers give a general historical overview of the disease in terms of survival, treatment strategies, and management. Though they may cite clinical cohort studies or treatment trials within their bodies, these papers were intended as broad educational overviews of HCC.

Llovet, Burroughs, and Bruix (2003) give a broad overview of HCC's incidence and treatment options. They note that HCC is one of the few cancers with well-defined major risk factors, and that this may help physicians in its early detection. Such early detection helps patients with so-called "Early HCC", defined as 2-3 nodules not exceeding $3 \mathrm{~cm}$, achieve excellent outcomes after OLT. These survival rates, along with many others including dropout rates, rates of survival for untreated control groups, intermediate stage, and advanced stage patients, as well as transplantation survival rates, are detailed in this paper's many statistical tables. These valuable survival rates were 
consulted in the development of this thesis's own case study (to be detailed in a later section). Additionally, this paper supported many of the assumptions inherent in this paper's case study: namely, the assumption of the bridging therapies available to the patient at every step in his treatment, and the assumption of the patient's liver function upon his entry of the model.

Parkin et al., (2001) provided global estimates of the burdens of all cancers worldwide. They note that HCC is the $3^{\text {rd }}$ deadliest cancer overall, and that its incidence rate (at the time of publication ) was 564.3 per 100,000 people. They provide this thesis with the assumption that patients who survive at least 5-years post-treatment can be considered cured, given the advanced age of most HCC patients, and that death after 5 years is likely to be from another cause. Furthermore, they model the shape of the survival curve within 5 years of treatment via the Weibull distribution.

Llovet et al., (2004) depict a diagnosis and treatment strategy for HCC, noting again the importance of early HCC treatment. Patients meeting the so-called "Milan Criteria" of 1 tumor not exceeding $5 \mathrm{~cm}$ or 3 tumors not exceeding $3 \mathrm{~cm}$ have excellent 5 year survival rates of 50-75\%. In their treatment group, they were able to diagnose $40 \%$ of their patients early. They note that there is no acceptable treatment for advanced-stage HCC patients, and that this treatment group should be given palliative therapy to ease their pain. Citing ethical reasons, they claim that a "control" group of patients has not been established. D-MODT's ability to simulate clinical cases in the theoretical realm and establish such a group will be discussed later in this paper.

Altekruse, McGlynn, and Reichman (2009) studied the rising incidence of HCC in the United States, noting that it tripled from 1.6 per 100,000 to 4.9 per 100,000 
between 1975 and 2005. However, the 2-4 year survival rates associated with HCC doubles between 1992 and 2004. This is due, the authors posit, to better awareness of the disease, more frequent HCC screening, and more aggressive therapeutic approaches such as OLT and resection. They note that Hepatitis-C, alcohol abuse, and obesity are among the leading factors associated with HCC infection.

El-Serag (2004) provided this thesis with several statistics on survival rates for different therapies and the overall disease. He notes that of the 8,500-11,500 new cases annually in the U.S., $74 \%$ occur in men, and their average age is 65 years. Referring back to obesity as having a strong association with HCC, the author states that the U.S. is an "epidemic of overweight and obesity," as $66 \%$ of adults are overweight and $20 \%$ are obese.

Bruix and Sherman (2010) describe many of the therapeutic options for HCC treatment and their associated survival rates. Additionally, this paper explains in detail the debate behind the use of MELD for liver allocation and its shortcomings when applied to HCC. This will be discussed in detail later in this thesis. Furthermore, the authors promote RFA as an excellent bridge, as it has similar efficacy to percutaneous ethanol injection (PEI), but requires far less sessions. The authors also promote sorafenib as the firstline treatment for patients who can no longer be treated with bridges such as TACE, RFA, or PEI. Highlighting the benefits of early detection and treatment, they note that, "In the past decades HCC has gone from being an almost universal death sentence to a cancer that can be prevented, can be detected early, and can be cured with appreciable frequency given early detection." 
Wiesner et al., (2003) further discuss MELD’s capability for ranking potential recipients with liver disease (HCC and otherwise). They claim that for the entire liver disease cohort, a subset of which is HCC patients, MELD accurately predicts 3 month mortality. A discussion of MELD and its use within this thesis's model will come later in this text.

The above papers served as a motivation for developing a tool to assist decisionmakers with the complex problem of HCC treatment. Given the disease's wide impact and positive response to the early administration of therapies, it is vital that technology be developed to aid physicians in prescribing the right treatments at the right times.

\subsection{Clinical Literature Review}

The following papers detail clinical studies that examine the effects of different approaches upon actual patient cohorts, as opposed to the hypothetical cohorts dealt with in other (i.e., Markovian modeling) approaches. Many of these studies compare one treatment versus another, or the effects of a singular treatment upon a large cohort of patients over time.

Azoulay, et al. (2002) sought to determine factors affecting the outcomes of retransplantation, and identified three variables associated with poor patient outcomes: urgency of re-transplantaition, age, and creatinine levels (one component of MELD). This paper contains survival rates for the years following transplantation and retransplantation (which are markedly lower). The authors conclude that re-transplantation 
denies allocating livers to patients who haven't undergone their first transplant, and that it is significantly more costly than the first transplantation.

Roberts, et al. (2010) studied the outcomes of patients who undergo ablation and then wait until transplantation ("ablate and wait") versus those patients who go through the transplantation procedure according to the Milan Criteria. They argue that the patients' underlying tumor biology is more apparent in scans for the former group, and that the Milan Criteria are "surrogate markers" for such biologies. They found that patients from the "ablate and wait" group had excellent outcomes post-OLT, but that $30 \%$ dropped out during the waiting period. The authors suggest that perhaps all patients should undergo a minimum wait time (e.g., 6 months), since treated tumors do not frequently degenerate in the time between a bridge and OLT.

Rodriguez-Luna, et al. (2004) studied the recurrence of Hepatitis-C (HCV) in patients post-OLT. They tested the hypothesis that $\mathrm{HCV}$ recurrence is more common post-LDLT versus DDLT, and found that there was no significant difference.

Sangiovanni, et al. (2006) performed a large cohort study in which they studied, in part, the development of $\mathrm{HCC}$ in $\mathrm{HCV}$ patients. Of the 214 patients studied, $32 \%$ developed HCC, and it was the main cause of death in $44 \%$ of these cases. After the diagnosis of HCC, these patients had an annual mortality rate of $31.5 \%$ per year. This is especially important to note since $\mathrm{HCV}$ is predicted to rise in incidence over the next decade.

Hashikura, et al. (2001) analyzed a center's 10-year history with 110 livingrelated donor graft transplantation (LRLT). This procedure was developed as an alternative to DDLT due to the serious shortage of donor livers. The authors found that 
this procedure offered excelled $(80 \%+) 1,3$, and 5-year survival rates for patients and grafts, and promote the expansion of LRLT.

Markmann, et al. (1997) examined the effects of 356 re-transplantations at UCLA from 1984-1996. Their results were in harmony with Azoulay, et al. (2002), in that the survival of the re-transplanted patients was inferior to those receiving their first OLT, and that the procedure of re-transplantation is an inefficient use of the already scarce number of donor organs.

Livraghi, et al. (1995) compared 3-year survival rates of cirrhotic HCC patients who had been treated with resection, PEI, and those who had not received any treatment. They found that resection and PEI offered 79\% and 71\% 3-year survival, respectively, compared to the $26 \%$ survival for the "do nothing" group. They concluded that surgery and PEI improve survival, and that more work needs to be done to determine which treatment to give patients.

Mazzaferro, et al. (1996) performed a cohort study of 48 cirrhotic patients with small (i.e., meeting the Milan Criteria) HCC who underwent OLT. After four years, the actuarial survival rate of these patients stood at $75 \%$, and recurrence-free survival was $83 \%$. They concluded that OLT is an effective treatment for unresectable Milan HCC patients with cirrhosis.

Llovet, et al. (2008) studied the effects of administering sorafenib to 602 patients with advanced HCC. They note that, in these advanced patients, no systematic therapeutic sequence had been established. This study found that sorafenib offered 10.7 months of median survival, compared to the placebo's 7.9 months. This additional lifetime of 3 months provided by sorafenib was built into this thesis's model. 
Chok, et al. (2011) performed a comparative study with 91 unresectable HCC patients who had received either TACE or RFA. Using only the objective of survival, they found that TACE survival was $80 \%$ and $58 \%$ after 1 and 2 years; RFA survival was $82 \%$ and $72 \%$ after the same times.

Lau and Lai (2009) reviewed the role of RFA in the treatment of HCC.

Reviewing a number of databases from 1997-2008, they argue that RFA is an excellent bridge for patients who expect to wait for a long time ( $>6$ months) until OLT. They provide tables with survival rates and dropout rates, which this thesis consulted in its case study.

Many studies in the literature focus on a single bridging therapy (e.g., TACE or RFA) and provide survival and dropout statistics for varying time periods. These papers were extremely variable as data sources for this thesis's case study. In particular, Choi, et al. (2007), Lu, et al. (2005), Mazzaferro, et al. (2004), Raut, et al. (2005) performed cohort studies on dropout rates and survival associated with RFA; Maddala, et al. (2004), Takayasu, et al. (2006), Oldhafer, et al. (1998), and Graziadei, et al. (2003) did so for TACE.

The above papers shed light on the state of the art of administering HCC treatments, and were invaluable resources in the design of the model described in this thesis. Survival statistics, dropout rates, etc. were consulted from these and other papers in the formulation of the case study elsewhere in this paper. 


\subsection{Markov Models: Overview and Clinical Studies}

All models are built to answer questions. In the previous section, the studies that were described reviewed the effects of various therapies on actual cohorts of patients to draw conclusions about the efficacies of various treatment strategies. However, many researchers wish to quickly simulate the results of a particular drug's application to a large group of patients without having to physically carry out the research over many years' time. To this end, many studies build models, make assumptions, and use prior data to simulate treatments, test hypotheses, and answer questions. Building models allows researchers to test therapeutic combinations that would be infeasible or unethical to test in the real world, and also allows for years' worth of trials to be simulated in mere moments.

In studies where the administration of a therapy is performed upon a large hypothetical cohort of patients, Markov models are used the vast majority of the time. The mathematics of these models are governed by the titular Markov Property, which states that for random variables $X_{1}, X_{2}, \ldots X_{n}$ and states $x_{1}, x_{2}, \ldots, x_{n}$, that:

$$
P\left(X_{n}=x_{n} \mid X_{n-1}=x_{n-1}, \ldots, X_{2}=x_{2}, X_{1}=x_{1}\right)=P\left(X_{n}=x_{n} \mid X_{n-1}=x_{n-1}\right)
$$

That is, the present state of a random variable depends only on the prior state and is "memoryless" of the past states. The following paragraphs detail many studies that have employed Markov modeling techniques. A discussion regarding Markov models' applicability and appropriateness is to follow. 
Llovet, et al. (2002) built a Markov model to assess the cost effectiveness of bridging therapies for cirrhotic HCC patients while awaiting OLT. The set of therapies considered was PEI, surgical resection, and no action. After bridging therapy, the patients could transition to OLT, dropout, or death (the same transition states used in this thesis post-bridge). States of health were analyzed in three-month cycles, and the model was simulated over the course of 10 hypothetical years. Employing a cost-effectiveness cutoff value of $\$ 50,000 / 1$ year life gained, the authors found that both PEI was a costeffective bridge for early HCC patients on the waitlist for OLT. Resection was costeffective should the patient face a longer wait time ( $>1$ year).

Cheng, et al. (2001), in their study of LDLT versus DDLT for small nonresectable HCCs, developed a Markov model to simulate the effects (in terms of survival) among three strategies: no OLT, intent to perform DDLT, and LDLT. Even when varying via Monte Carlo simulation many factors (e.g., tumor grown pattern, severity of cirrhosis, regional transplant volume, etc.), they found that LDLT dominated DDLT in terms of life expectancy. This paper did not consider the monetary costs associated with either procedure.

Sarasin, et al. (2001) also compared the effects of LDLT versus DDLT with their own Markov model. Unlike the previous paper, this study considers the costs of both types of transplantation. For their model's hypothetical patient, the authors found that LDLT was more effective than DDLT after 3.5 months on the waiting list. For waiting times of 1 year, LDLT provided up to 2.8 more years of survival than DDLT.

Northup, et al. (2009) developed a multistage Markov model to explore the costs and benefits between three strategies: medical management only, waitlist for DDLT, 
waitlist with possible DDLT or LDLT. The study was simulated over a hypothetical period of 10 years. The authors found that both strategies 2 and 3 were cost-effective over the 10-year period compared to strategy 1 . Regarding the tradeoffs between strategies 2 and 3, the study found that the inclusion of LDLT to a DDLT program does offer better patient survival, albeit at an increased cost.

Volk, Vijan, and Marrero (2008) built a Markov model to compare transplantation's survival benefit for patients who fall outside of the Milan Criteria versus the harm to other patients on the waitlist. They note that, as of their study, no empirical research had defined the post-transplant survival rates that would justify expanding the Milan Criteria. This study used the UCSF Criteria (1 tumor not exceeding $6.5 \mathrm{~cm}, 3$ tumors not exceeding $4.5 \mathrm{~cm}$ ), and their Markov model examined the decision of whether or not to perform OLT on a patient who exceeds the Milan Criteria but meets the UCSF Criteria. They found that transplanting patients who exceed the Milan Criteria resulted in a $44 \%$ increased risk of death, and concluded that this outweighed the benefit of OLT. They note that any expansion of the Milan Criteria will have to be supported by excellent survival rates for the newly admitted patients.

The work of Naughler and Sonnenberg (2010) was heavily consulted in the development of my model. In their paper, the authors constructed a Markov chain to compare survival rates and the cost-effectiveness of different HCC pre-OLT treatment strategies for patients with small $(<2 \mathrm{~cm})$ HCC. The two strategies considered were: immediate TACE or RFA, and monitoring alone. Separate Markov chains were built for the simulation of each strategy, and a hypothetical cohort of 1000 patients was studied over 10 years. The authors concluded that it was more cost-effective (their threshold was 
$\$ 50-100 \mathrm{k} /$ year) to bridge with TACE/RFA than to simply monitor, and that RFA was a better bridge than TACE (although some patients are precluded from RFA due to technical issues). Aside from insight on the tradeoffs between TACE/RFA, this paper provided this thesis with several valuable survival and cost statistics for the therapies considered, and also an exponential mathematical equation used for calculating monthly rates of transition from yearly rates.

Vitale, et al. (2010) developed a Markov model to study the efficacy of employing sorafenib as a bridging therapy towards OLT versus using no bridge for 6 months. The authors found that Sorafenib provided a median survival time of 94 days, which was consistent with other papers and has led to this thesis's assumption of 3 months of provided lifetime.

\subsubsection{Limitations of Markov Models}

As evidenced by their presence in the papers reviewed in the prior section, Markov models are dominant in the literature. This is due, in part, to their attractive simulation ability. Software exists (e.g., Data 3.5, Tree Age Software) that allows medical researchers to quickly input probabilities, generate, and solve Markov models in a small amount of time. In addition, the passage of time within the model is very conveniently handled by the Markov mathematics, as each transition from one state to another is analogous with the passage of a homogenous amount of time. In the papers reviewed, this period was almost always three months.

Despite their attractiveness and convenience, the Markov approach has its shortcomings. Davis, et al. (2010) note that, "[Markov] models have significant 
limitations in that the studied cohort is considered homogeneous and traverses through their disease at a fixed and predictable rate over time." To his first point regarding the homogeneous cohort, the papers this thesis reviewed did indeed assume that every patient inputted into their Markov model was the same. As an example, Cheng, et al. (2001) assumed a homogeneous cohort in which every patient was a 58-year-old male with cirrhosis and a single non-resectable $3.5 \mathrm{~cm}$ HCC. In practice, the uniqueness of each patient and his initial state of health are key in determining which therapies a physician prescribes. The costs and life expectancies, and subsequently a model's output, associated with different therapeutic sequences differ greatly depending on these initial states of health. Thus, a more appropriate model would consider that every patient could have his own unique characteristics and initial state of health; that is, it would allow for a heterogeneous and diverse patient population.

The homogeneous passage of time between states in the literature's Markov models is also an assumption that must be called into question. Formally, time homogeneity is satisfied if, for states $i, j$, time $t$, and step $s$ :

$$
P\left(X_{t}=j \mid X_{t-1}=i\right)
$$

is independent of $t$. Several papers such as that by Llovet, et al. (2002) assume that three months pass between every state transition in their model. At UVA, the clinical team does not necessarily wait three months between every checkup; a patient is scanned one month after his initial treatment is administered, then every three months (if he remains on the same therapy) or one month (if he changes therapies). Additionally, a patient may 
respond differently to therapies at different times throughout his treatment, so the independence of $t$ in Equation (2) is further unjustified. A more appropriate model should consider all combinations of this one-and-three month checkup lattice, and not assume that, for example, a patients state of health will behave the same way from months 3 to 6 as it would from months 6 to 9 .

Perhaps the greatest assumption of all is the "memorylessness" property described previously in Equation (1), in which the current state of a system depends only on the previous state. Surely, a physician examining his patient at his $5^{\text {th }}$ checkup would not base his current therapeutic recommendation solely on the $4^{\text {th }}$ checkup, but rather use all available past and historical information so as to make the most informed decision. The Markov property ignores this past information, and disregards the chain of events that lead to the system's prior state. A more appropriate model would allow for a current decision to incorporate all knowledge of past events.

The above critiques are not meant to dismiss the Markov modeling approach, but rather to identify ground upon which complementary and supplementary models can be constructed. The remainder of this thesis describes the methodology behind a complementary Bayesian model. 


\section{METHODOLOGY}

The following text details the particular methodological aspects that comprise the multi-objective decision tree (MODT) and later the dynamic multi-objective decision tree (D-MODT), the product of this thesis. The structure and development of MODT is explained, and an overview of Bleistein's (2011) MODT for HCC treatments is presented.

\subsection{Decision Theory}

The decision tree, popularized by Raiffa's (1968) seminal publication, is both analytically and graphically informative, as it employs Bayesian analysis to provide the decision-maker with the expected value of the objective while visually depicting all stages in the decision process. A sample decision tree is depicted below in Figure 1. 


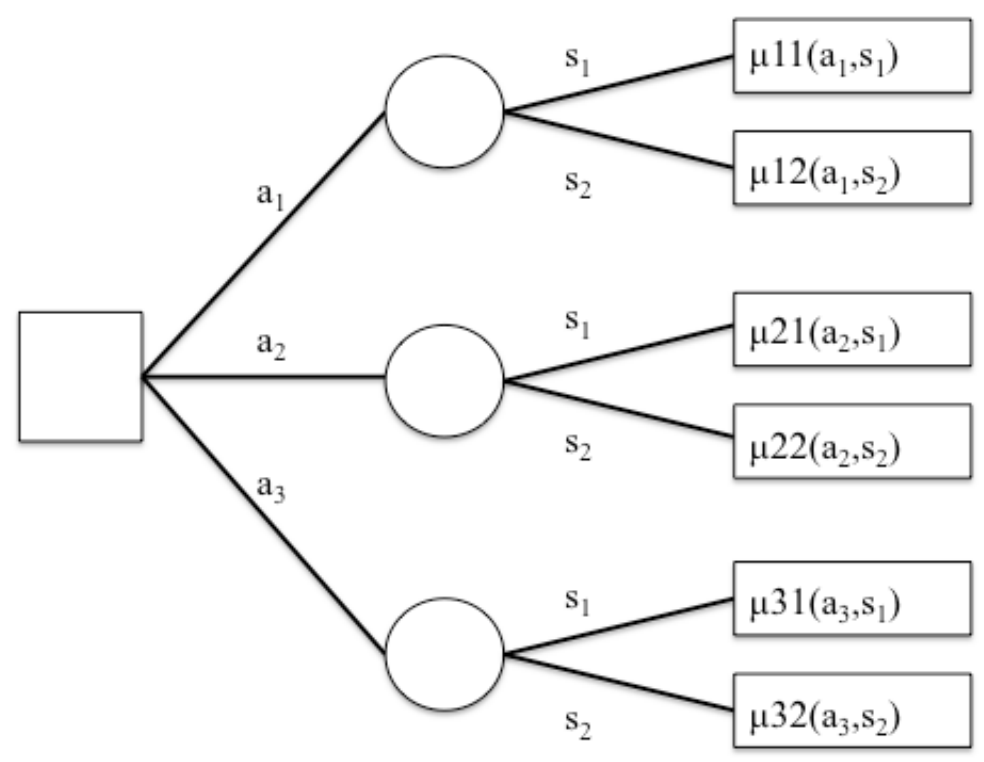

Figure 1: Single Objective Decision Tree (Haimes 2011)

Decision tree analysis contains three basic components:

1: Decision nodes (represented by squares), at which point the decision-maker must select one action (represented by a line) among a set of feasible alternatives

2: Chance nodes (represented by circles), which represent points at which divergent probabilistic states of nature (represented by lines) occur

3: Consequences: at the end of each branch, the value of the outcomes (i.e, cost) is denoted by $\mu$.

Multiplying the probabilities associated with each chance branch by their corresponding consequence $\mu$ and then averaging these values out for each chance node solves the decision tree. Because of the mathematical similarities between this and the MODT/D-MODT, a formal discussion of the mathematics behind the solution procedure will occur later in this thesis. 


\subsection{Multiple Objectives}

Although single-objective decision trees such as those theoretically depicted in Figure 1 are a powerful graphical and analytical tool for decision-makers, most realworld problems are characterized by multiple, non-commensurate, and conflicting objectives (Haimes 2011). The description of these objectives as non-commensurate, by definition, should dissuade modelers from employing approaches that seek to combine multiple objectives into a single scalar value. Yet, as is evidenced in the review of the literature, many researchers (Llovet, et al. (2002); Naughler and Sonnenberg (2010); etc.) optimize in terms of cost-effectiveness, and by doing so combine the competing objectives of minimization of cost and maximization of life into one scalar value. Thus, it is necessary to consider a method where the values of objectives are kept separate rather than combined. The concept of Pareto-optimality allows decision-makers to view tradeoffs among different strategies while still maintaining the separateness of the objective functions. It also provides the decision-maker with a set of feasible solutions rather than a singular "optimal" solution. In plain English, a solution to a multiobjective optimization problem is termed noninferior, or Pareto optimal, if improving one objective function can only be achieved at the expense of degrading another. To define a Paretooptimal solution mathematically, consider the following multiobjective optimization problem (MOP):

$$
\begin{gathered}
\min _{x \in X}\left\{f_{1}(\boldsymbol{x}), f_{2}(\boldsymbol{x}), \ldots, f_{n}(\boldsymbol{x})\right\} \\
X=\left\{\boldsymbol{x} \mid g_{i}(x)<0 ; i=1,2, \ldots, m\right\}
\end{gathered}
$$


where in Equation (3) $\mathbf{x}$ is an $n$-dimensional vector of decision variables, $X$ is the set of all feasible solutions, and $\mathrm{g}_{\mathrm{i}}(\mathbf{x}) \mathrm{s}$ the $i$ th constraint.

A decision $\mathbf{x}^{*}$ is said to be Pareto-optimal to the system posed by Equation (3) if and only if:

$$
\nexists \bar{x} \ni f_{j}(\bar{x}) \leq f_{j}\left(x^{*}\right) ; j=1,2, \ldots n
$$

with strict inequality holding for at least one $j$. To clarify, consider the following hypothetical case in which we seek to minimize two objective functions. Suppose we have the following three options:

$$
\left[\begin{array}{l}
f_{1} \\
f_{2}
\end{array}\right]=\left[\begin{array}{l}
80 \\
20
\end{array}\right]_{1},\left[\begin{array}{l}
20 \\
80
\end{array}\right]_{2},\left[\begin{array}{l}
75 \\
90
\end{array}\right]_{3}
$$

We would say that strategy 3 is dominated by strategy 2 , since in terms of the objective (minimize both $\mathrm{f}_{1}$ and $\mathrm{f}_{2}$ ) it is inferior. Similarly, we would say that strategies 1 and 2 are Pareto-optimal with respect to the solution set since improving one objective function (e.g., reducing $f_{1}$ from 80 to 20 ) can only be done at the expense of degrading another (e.g., increasing $\mathrm{f}_{2}$ from 20 to 80 ). Graphically, we can draw a Pareto-optimal frontier for the example as follows: 


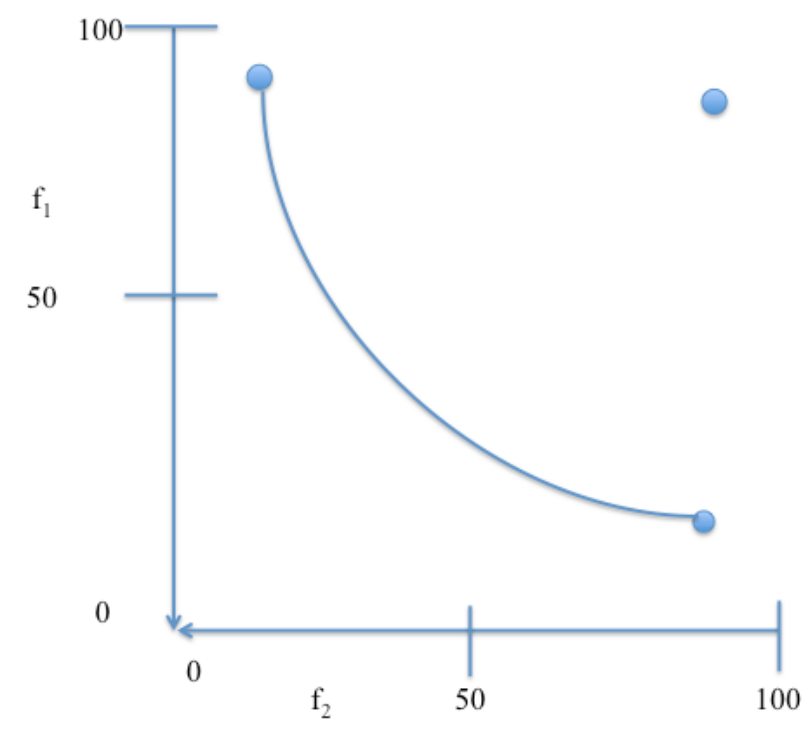

Figure 2: Sample Pareto-Optimal Frontier

The above example illustrates that the concept of Pareto-optimality allows the decisionmaker to decide among many feasible solutions, rather than force him to weight objectives and be confined to one "optimal" point. In the MODT methodology, the model output is a set of Pareto-optimal strategies that can be traced throughout the tree, all the way back to the first decision. What follows is a brief overview of the MODT methodology.

\subsection{Multi-Objective Decision Trees (MODTs)}

\subsubsection{Overview}

Haimes, Li, and Tulsiani (1990) extended the single objective decision tree (SODT) models prevalent in earlier decades to broaden the concept of decision-tree analysis, since most real-world problems have multiple rather than single objectives. Thus, the final branches of the MODT contain vector-valued performance measures, 
rather than scalars. The MODT's model structure is akin to that of the SODT, in that it is a sequence of decision nodes and probabilistic chance nodes. Compare the topology of the SODT in Figure 1 with that of the MODT in Figure 3:

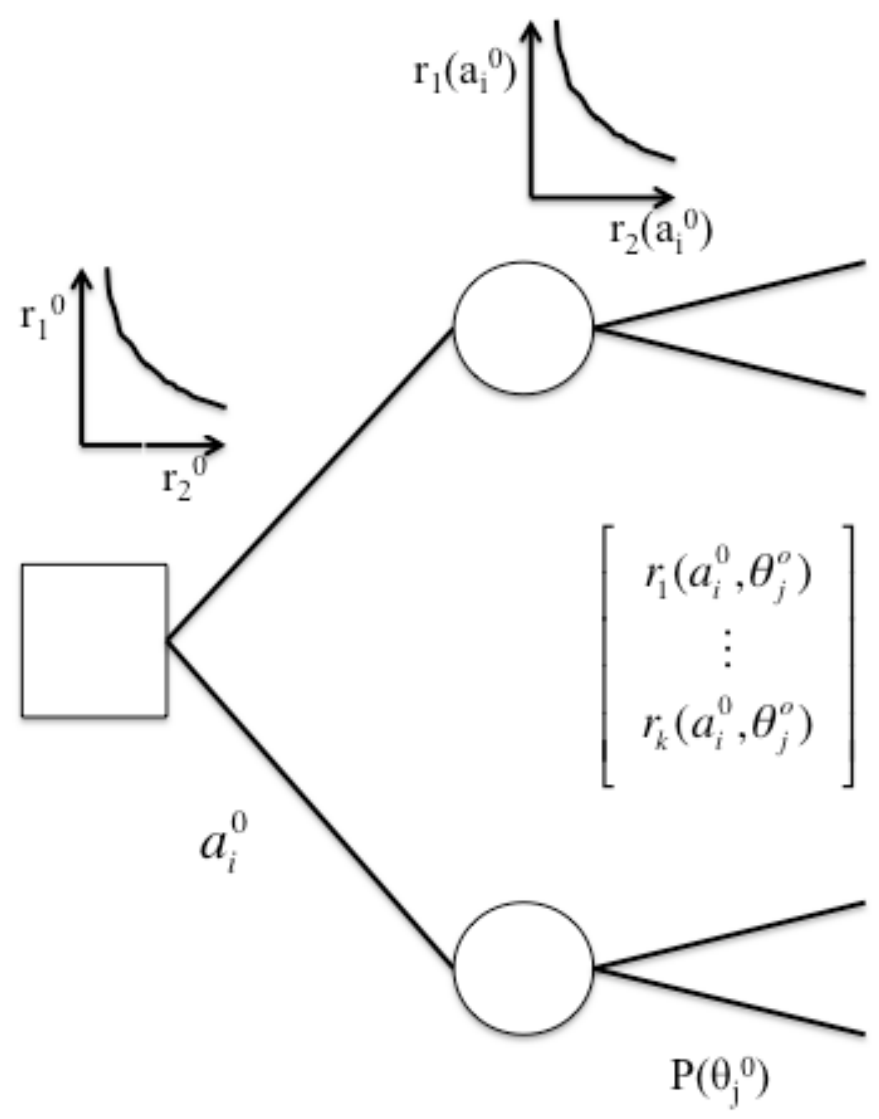

Figure 3: Structure of MODT (Haimes, Li, and Tulsiani 1990)

The notation in the above figure corresponds to the following:

1) $a_{i}{ }^{n}$ is the $i t h$ action available to the decision-maker at decision node $n$

2) $\theta_{j}^{n}$ is the $j t h$ probabilistic state of nature possible at chance node $n$

3) $r\left(a_{i}, \theta_{j}\right)=\left[r_{l}\left(a_{i}, \theta_{j}\right) \ldots, r_{k}\left(a_{i}, \theta_{j}\right)\right]^{t}$ is the $k$-dimensional vector-valued

performance measure associated with action $a_{i}$ and probabilistic state of nature $\theta_{j}$ 
From the above definitions, the general definition of Pareto-optimality for MODTs is the following (for minimization of all objective functions):

A point $r=\left[r_{1}, r_{2}, \ldots, r_{k}\right]^{t}$ in the objective function space is said to be Paretooptimal if there does not exist another feasible point $r^{\prime}=\left[r_{1}{ }^{\prime}, r_{2}, \ldots, r_{k}\right]^{t}$ such that $r_{i}{ }^{\prime} \leq r_{i}$, for $i=1,2, \ldots, k$ with at least one strict inequaility holding for $i=1,2, \ldots, k$.

\subsubsection{Solution Procedure}

The following mathematical approach is taken from Haimes (2011). The solution procedure for MODTs begins at the end (leftmost branches) of the decision tree and proceeds iteratively until the initial decision node of the tree is reached. At each decision node $n$, and at each branch emerging to the right side of that node, there is a corresponding set of vector-valued performance measures $r\left(a_{i}^{n}\right)$ for each alternative $a_{i}$. We identify the set of noninferior solutions by solving $r^{n}=\min \mathrm{U}_{i} r\left(a_{i}^{n}\right)$, where $\mathrm{U}$ is the union operator. At each chance node $m$ and at branches emerging to the right of that node, we find the corresponding set of vector-valued performance measures $r_{j}^{m}$ for each state of nature $\theta_{j}^{m}$. We then calculate the vector-valued performance measures denoted $r^{m}=\min E_{j}^{S}\left\{r_{j}^{m}\right\}$. This procedure is repeated until the starting point of the tree is reached. 


\subsubsection{MODT Analysis for HCC Treatments}

Bleistein (2011) employed the MODT methodology to examine the tradeoffs between various treatment strategies for HCC. He built a simplified model, which this section will briefly review. This served as motivation for this thesis's extended dynamic methodology, and the two approaches will be greatly compared in the later sections of this paper.

Bleistein built a two-stage model, depicted below in Figure 4, to examine the tradeoffs associated with different treatment strategies.

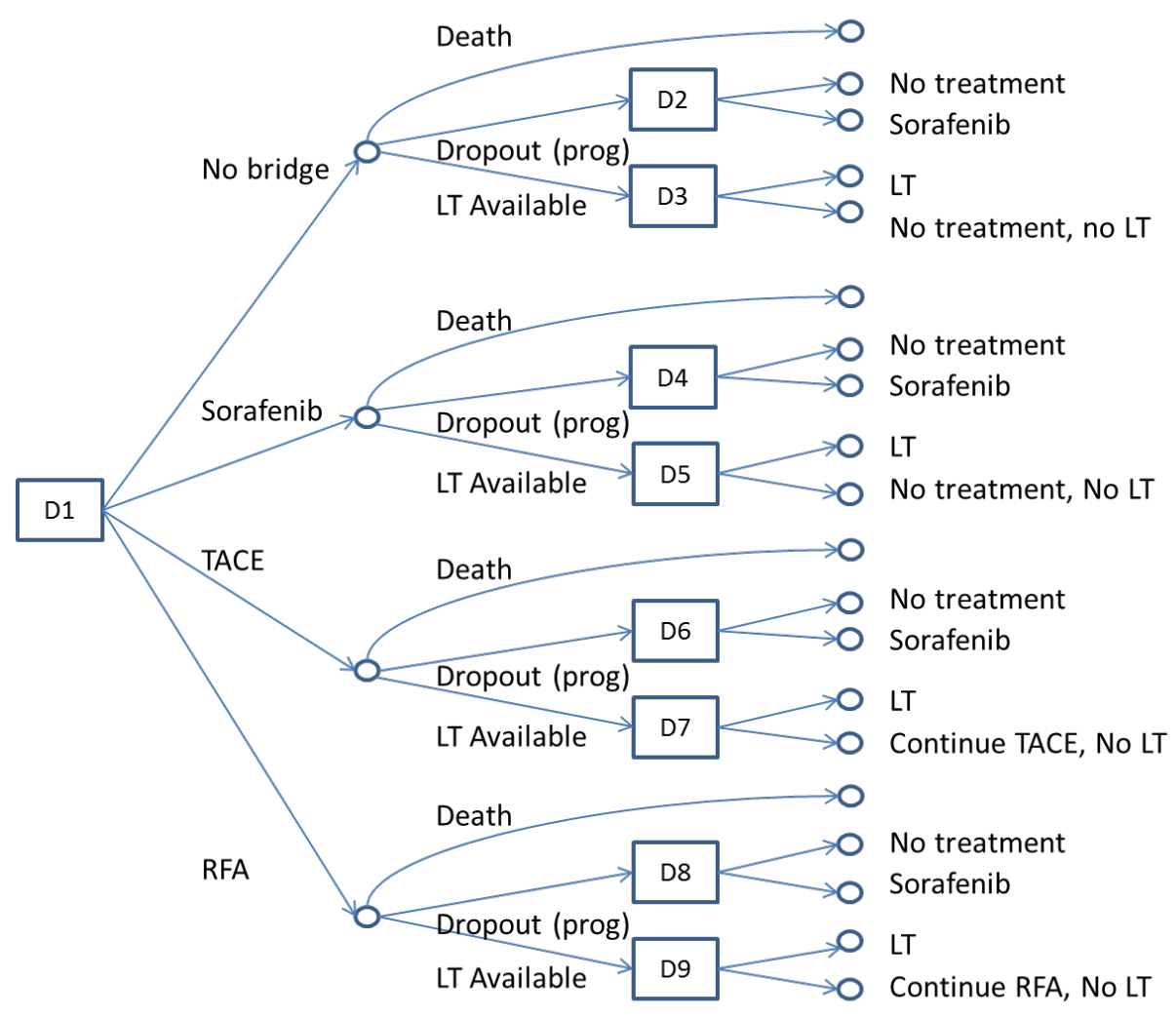

Figure 4: Simplified MODT for HCC (Bleistein, 2011) 
This model spanned two decision periods: the decision of which pre-operative bridging therapy to employ, and the decision of whether or not to transplant (if the patient was eligible for OLT) or which post-dropout therapy to employ (if the patient was removed from the wait list). This decision tree considered two objective functions: maximize the patient's additional expected life (in terms of years of life), and minimize the patient's expected cost of therapy (in terms of \$U.S.). Bleistein considered TACE, RFA, and "Do Nothing" as bridging therapies for the initial decision node. For the chance node associated with the results of the bridging therapy, the patient either died, dropped out from the waiting list, or remained on the waiting list as an OLT candidate until a liver became available. In the case that he dropped out from the waiting list, the patient could either be prescribed sorafenib or nothing. In the case that the patient remained on the waiting list, he either received an OLT eventually, or continued his bridging therapy (from the first decision node) without receiving an OLT.

To populate his model, Bleistein derived various probabilities such as dropout rates, death rates, transplant rates, and post-transplant survival from the UNOS database. He noted that, more often then not, there was not sufficient data in the UNOS database (such as probabilities for survival within smaller time periods), so he consulted the literature in many instances. These probabilities, the techniques with which he harvested them, and his modeling assumptions were all invaluable towards this thesis as well as for the population of the model in its case study.

Solving his model, Bleistein found that the Pareto-optimal treatment sequences were grouped into two categories: those that were characterized by high additional lifetime at high cost to the patient (i.e., eventual transplantation), and those that were 
characterized by low additional lifetime but at low cost to the patient (i.e., where transplantation was not carried out). Again, Pareto-optimality does not prescribe a cost per years of life threshold as is done many other modeling approaches, so it is up to the physician (and the patient) to decide whether the high life/high cost or less life/less cost option is right for them. Regarding the bridging therapies, Bleistein went on to discuss the many cases (depending on a patient's progression, etc.) where one therapy would dominate another. It is important to note that in his (and the current) model, input data was combined from many sources, and many assumptions were made. He admits that altering these assumptions could drastically change the output of the model. Thus, it is important to regard the purpose of Bleistein's work as laying the foundation for a new modeling approach rather than solely as a study to determine optimal therapeutic sequences.

Although Bleistein's model was groundbreaking in that it presented a novel approach to modeling HCC treatments, it was quite simplified and included many limiting assumptions. The next section describes the purpose of this thesis: the development of a more appropriate, dynamic model. 


\section{DYNAMIC MULTIOBJECTIVE DECISION TREE FOR HCC}

The following subsections detail the motivation behind and the development of a dymanic multi-objective decision tree (D-MODT) for HCC treatments. A large-scale methodology for producing patient-specific D-MODTs is discussed, and a simplified three-month D-MODT is charted and populated with data from multiple sources.

\subsection{Motivation}

The catalyst for the development of the present work was Bleistein's (2011) preliminary work with developing a simplified MODT for HCC treatments. In his work, he identified several major areas for the improvement of his model. These and other potential areas for improvement of his approach are discussed in the following paragraphs:

Consideration of time. Haimes (2012) highlights the centrality of the time frame in all decision-making, and that models must answer the following question: What are the impacts of current decisions on future options? The time frame is critical in healthcare delivery, and should be explicit in a model that seeks, among other things, to assess the impacts of various treatment strategies over the course of $\mathrm{HCC}$ treatment. Information regarding for how long a patient is prescribed a specific therapy, how long he is on that therapy before he is switched to another therapy, how long he must wait for an OLT, etc. 
may greatly vary the expected life and cost associated with his treatment. Bleistein's MODT was time-invariant in that, in his model, a physician selected (and was confined to) one pre-transplant therapy. Yet, as the goal of a bridging therapy is to maintain a patient's state of health as he awaits transplantation, a more appropriate model would consider the possibility that the physician may decide upon a different therapy at every possible decision juncture. Indeed, the Bayesian updates that mathematically characterize MODT methodology suggest that a methodology in which patient probabilities of progression, death, etc. can be updated when every new piece of information is received (at every checkup) would be more a more appropriate approach. At every chance node, the patient's probability of progression, etc. should be conditioned mathematically upon time, among other factors.

Additional treatment granularity. Bleistein realized that his tree could be greatly expanded in terms of pathways. His pre-transplant bridging decision node indicated upon which "path" a patient was put in terms of bridging strategy, but it did not provide information regarding for how long that therapy was administered, or at what point in his overall therapy it was prescribed. This presents an opportunity to add great patient specificity in the model: where Bleistein's model assumed that every patient who followed the TACE decision branch received the exact same treatment schedule while he awaited transplantation, a more realistic model could include many TACE branches corresponding to different possible TACE treatment times, etc. In addition, Bleistein's model did not consider the possibility that a TACE patient may be switched to RFA, or any other possible combination of therapies. In practice, surely a physician who observes 
a patient's poor response to his initial therapy will not continue to administer that same treatment. Proper population of such a refined model, in which not only combinations of therapies but also combination of times of administration are possible, could shed much light on the efficacies associated with times of treatment. The lattice-like and sequential nature of the MODT/D-MODT topology is convenient for answering such questions.

Additional outcome granularity. In Bleistein's model, the only three outcomes into which a patient could be classified post-bridging therapy were "death, dropout from waitlist, eligible for OLT'. By charting only these three chance branches, this makes the assumption that all patients who were still eligible for OLT once a liver became available would have the same benefit from receiving the liver (in terms of the objective function). Surely, not all patients who undergo OLT do so from the same state of health. A more appropriate model should separate this and other branches into more refined categories, such as "condition improved, condition stable, condition worsened," so that survival and cost could be better projected. Again, this calls into question the issue of the time domain. Rather than separating patients into a small number of categories that correspond to their state at the end of their bridging therapy, as Bleistein's model did, a more refined model would separate patients into a number of categories based on their current state of health as every new piece of information arrived. Yet, even the categories of "improved, stable, worsened" are ill-defined and necessitate a metric with which to classify a patient's condition of health. This goal introduces the need for the definition of a state variable, which will be discussed at length in a following section. 
Geographic specificity. Currently, UNOS has divided the United States into eleven different transplant regions. The expected waiting times until transplantation vary greatly in these regions; for example, a patient residing in Indiana (region 10) may wait for far less time for a transplantation that a patient residing in New York City (region 9). Thus, length of treatment and, by extension, the sequences of bridging therapies are different for different regions of the country. The expected survival and cost will differ greatly given a patient's length of treatment, and a more appropriate model will take as an input a patient's OPTN region so that it may accordingly account for his expected wait time until a liver transplantation becomes available.

Patient specificity. One of the critical weaknesses of Markov models is that they assume a homogeneous patient cohort, that is, they treat every patient who enters the model as the same (e.g., a 58-year old cirrhotic male with a $3.5 \mathrm{~cm} \mathrm{HCC}$ ). Bleisten's model, as well, did not adjust its output dependent on which patient entered the decision tree. However, the HCC population is quite heterogeneous, and a realistic model would account for the fact that every patient will respond differently to different treatments. Thus, such a model will project Pareto-optimal treatment sequences based off of not only the historic national databases, but also according to the specific patient's own current and past states of health, responses to prior treatments, etc. Again, this necessitates the definition of a state variable with which a patient's health can be traced and updated throughout his passage in the model. His state should be defined as he enters the model, updated at every checkup, and used to generate his expected life/cost vectors at every decision node 


\subsection{D-MODT Topology}

This section describes the structure of the D-MODT for HCC treatments, and is based off of the motivating factors in the previous section. Because the model is customizable for every patient based off of his state of health and his geographic region, there does not exists a "full-scale" model; rather, there exists a framework for developing a detailed model for every patient. This model can span many decision periods and become quite large very quickly, so for the purposes of readability the specific nodes themselves will be examined along with an explanation of how they are incorporated into a patient's individual mode. Perhaps the biggest limitation of such a refined and specific methodology is that data simply does not exist with which to populate all hypothetical branches of a patient's individual decision tree. After the discussion of the model structure, a simplified three-month case study will be examined, in which many assumptions were made so as to populate the model with data from multiple sources.

The D-MODT is comprised of sequences of decision and chance nodes. Akin to Bleistein's model, the D-MODT for HCC treatment has two decision "periods": the pretransplant bridge, and the decision at the time of transplant. Unlike his model, however, these periods are not characterized by a single decision node. Rather, they contain many possible decision nodes, based off of the length of the patient's time until transplant. Since a physician re-evaluates and re-scans his patient at every checkup, the D-MODT is partitioned so that there is a new decision node for every checkup. Recall the following timing of checkups discussed earlier, in which the wait between checkups is 3 months if the same bridging therapy is employed as the last checkup, and 1 month if the bridging therapy is switched. For example, suppose that at the patient's initial checkup (Month 0) 
after being placed on the wait list, he is prescribed TACE. Since this is a "new" bridging therapy, he is checked again in one month (at Month 1). At this Month 1 checkup, if the physician decides that the patient should remain on TACE, he is not checked again until Month 4 (the 3-month assumption), unless of course a transplant becomes available, at which point the patient would be immediately transplanted. However, if the physician decides to switch the patient's therapy to, for example, RFA, then the patient is checked again at Month 2 (the 1-month assumption). To illustrate this, a simple pre-transplant DMODT is presented in Figure 5, where only two hypothetical therapies (TA and TB) and two probabilistic states of health (B and $\mathrm{W}$ ) are considered.

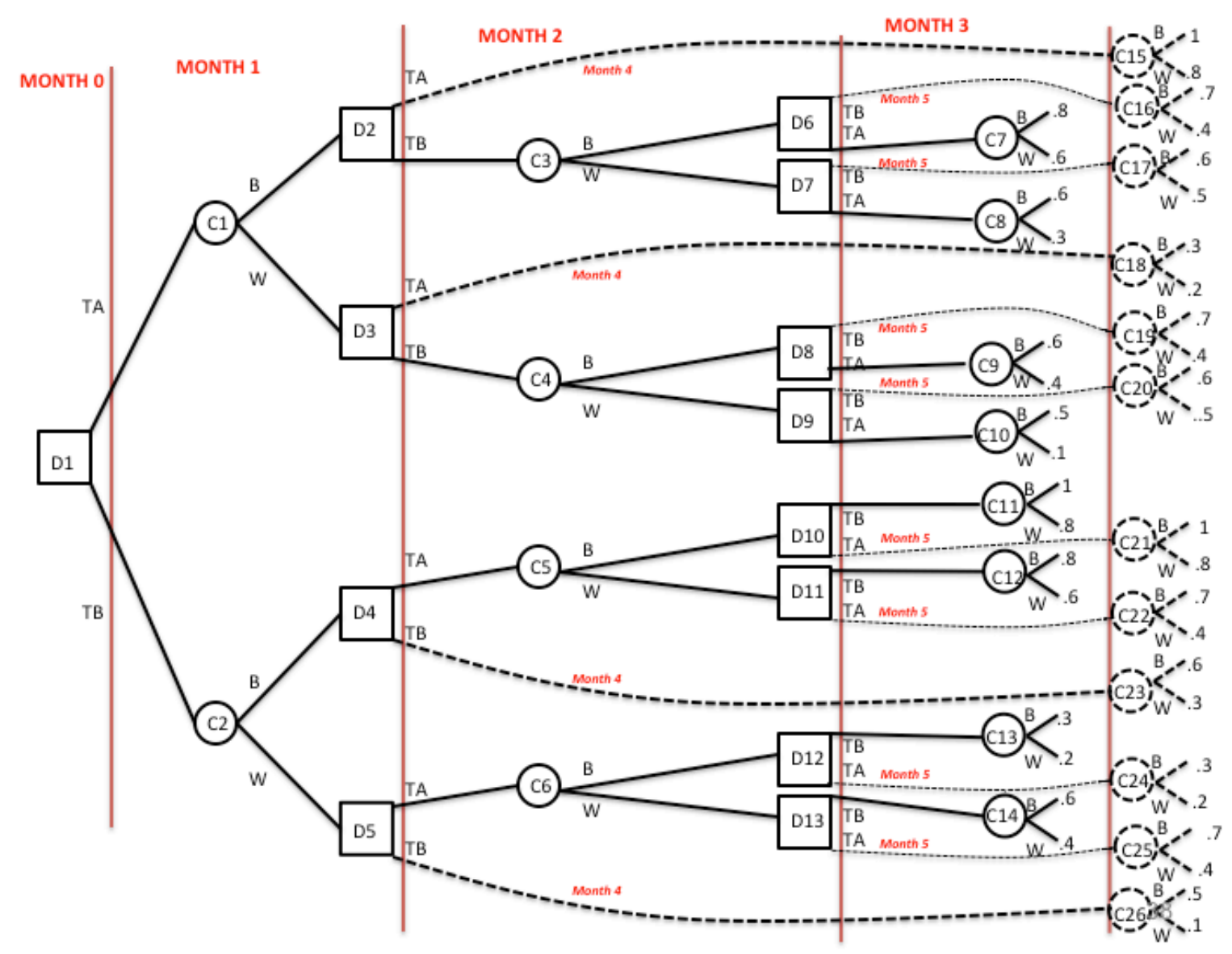

Figure 5: Simplified Pre-Transplant D-MODT 
Note that in Figure 5, the passage of every month is depicted by a horizontal line. It is important to note that, while this dynamic and sequential decision node structure is more realistic, it also very quickly can span a massive decision tree. Even in this very simplified example, there are already 40 potential paths through the decision tree.

The horizontal length (i.e., number of months) of the D-MODT is customized for each patient based off of his geographical location. The Organ Transplant and Procurement Network (OPTN) keeps up-to-date records online of the median wait times for patients dependent on their region (i.e., 1-11) and their initial MELD score at listing. These are the two inputs to the decision tree, which make it customizable for each patient. Appendix A contains a table of median wait times (in days) based off of initial MELD score by region, as well as $95 \%$ confidence intervals for these times. It is important to remember that although HCC patients are assigned priority scores of 22 points plus an additional 3 points every 3 months until a transplant event (i.e., OLT, death, dropout) occurs, that this is an assigned MELD score rather than his calculated MELD score, which is based off his levels of creatinine, bilirubin, and INR. That is, HCC patients effectively have 2 MELD scores at all times: that which prioritizes their place on the wait list for OLT (assigned), and that which corresponds to their three biochemical factors (calculated). These assigned MELDs of 22+ wait times vary among each region from 490 days (Region 5) to 27 days (Region 10). For each patient, his pretransplant D-MODT spans for the median number of months in Appendix A according to his initial assigned MELD score. For the hypothetical D-MODT in Figure 5, this median wait time would be 3 months. 
The next subsections detail the selection of the available therapies at each decision node and the states of nature emanating from each probabilistic chance node. A full sample D-MODT is too large to present in a readable format, and is presented in simplified form at the end of the section.

\subsubsection{State Variable and MELD Discussion}

The states of a system, commonly a multidimensional vector, characterize the system as a whole and play a major role in estimating its future behavior for any given inputs. Haimes (2012) highlights the centrality of the state variable, noting that every system is characterized at any moment in time by its state variables. One of the major weaknesses of Bleistein's model is that there was no central state variable with which the physician could gauge his patient's progress throughout the treatment. In a dynamic problem, it is vital that a state variable be defined, so that the system can be objectively compared to itself at different times throughout its evolution. A formal definition of "state variable" is given by Chen (1999):

The state $\mathrm{x}\left(\mathrm{t}_{0}\right)$ of a system at time $\mathrm{t}_{0}$ is the information that at time $\mathrm{t}_{0}$ that, together with the input $\mathrm{u}(\mathrm{t})$, for $\mathrm{t} \geq \mathrm{t}_{0}$, determines uniquely the output $\mathrm{y}(\mathrm{t})$ for all $\mathrm{t} \geq \mathrm{t}_{0}$.

Thus, the prescription of any therapy to a patient by the physician is done so to change (i.e., improve) the state of the patient's health. In order for him to prescribe said therapy, he must know the patient's current state of health. Thus, a major obstacle in this thesis was the selection of a state variable with which to accurately gauge the condition of the 
patient at any time throughout his treatment. Many options were considered, including MELD score, Alpha-fetoprotein levels, magnitude of tumor volume between scans (i.e., $\%$ regression, progression), etc.

Alpha-fetoprotein (AFP) has been used as a prognostic tool for HCC for some time (Nomura, Ohnishi, and Tanabe, 1989). Kulik, et al. (2012) performed a cohort study on the outcomes of LDLT and DDLT recipients with HCC and found that AFP levels post-therapy were good predictors of survival post-OLT. However, this does not necessarily support AFP's use as a state variable throughout the patient's pre-OLT treatment sequence.

MELD, devised in 2002, is a commensurate score of three biochemical factors which seeks to assess a patient's risk of death within 90 days of being placed on the wait list for OLT (Kamath and Kim, 2007):

$M E L D=3.78[$ Ln serum bilirubin $(\mathrm{mg} / \mathrm{dL})]+11.2[\mathrm{Ln} I N R]+9.57[$ Ln serum creatinine $(m g / d L)]+6.43$

At every checkup, the patient can be re-scanned, his MELD score re-calculated, and thus his risk of death updated. This suggests that MELD would be an appropriate state variable with which to gauge a patient's progress (the lower the MELD, the less risk of death). However, the fact that MELD is not observable is problematic: a state variable should be a single observable value (i.e., biochemical factor). A therapy should be prescribed based on a specific observable factor, not an aggregate or commensurate score. At the advice of UVA physicians, however, it was decided that MELD is the best 
currently available candidate for this model's state variable. The physician, however, should still be informed of the independent values of each of the three biochemical factors at each checkup. Thus, the identification and implementation of a more appropriate state variable is an avenue for the expansion of this work.

Having selected MELD as the state variable for the D-MODT, the question was then how to use MELD at the chance nodes to add granularity to possible patient responses to the therapy at the preceding decision node. Since MELD is an aggregate of three $\ln ()$ functions, it is a continuous score. While it is possible to assign continuous probability distributions at the chance nodes, many studies a la Merion, et al. (2005) instead use MELD “buckets," that is, they group patients into MELD categories of 2-3 points and study transitions between these buckets. Merion's paper contains information regarding the proportion of the wait list population in MELD buckets of 2 for initial listing as well as at the time of transplant.

For this work, it was decided to discretize MELD scores at every chance node in the following manner: $\{[0,10) ;[10,15) ;[15,20) ;[20,25) ; 25+\}$.

\subsubsection{Sequence of Nodes}

In the pre-transplant portion of the tree, the pathways emanating from every decision node correspond to potential therapies at that decision node. At the initial decision node, and at every "bridging therapy" decision node, the following options are available and are displayed below in Figure 6: 


\section{Pre-Transplant D-MODT: Bridging Decision Nodes}

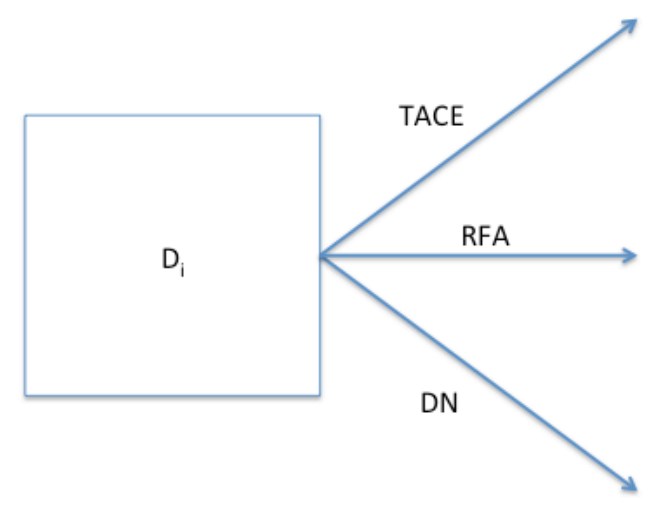

Figure 6: Initial and Bridging Decision Nodes

1. TACE: Transarteral chemoembolization

2. RFA: Radiofrequency ablation

3. DN: "Do nothing" i.e., no bridging therapy

In lieu with the methodology of MODT, a chance node follows every decision node in the D-MODT methodology. Every chance node has the following pathways emanating from it, and is depicted below in Figure 7: 


\section{D-MODT Chance Nodes}

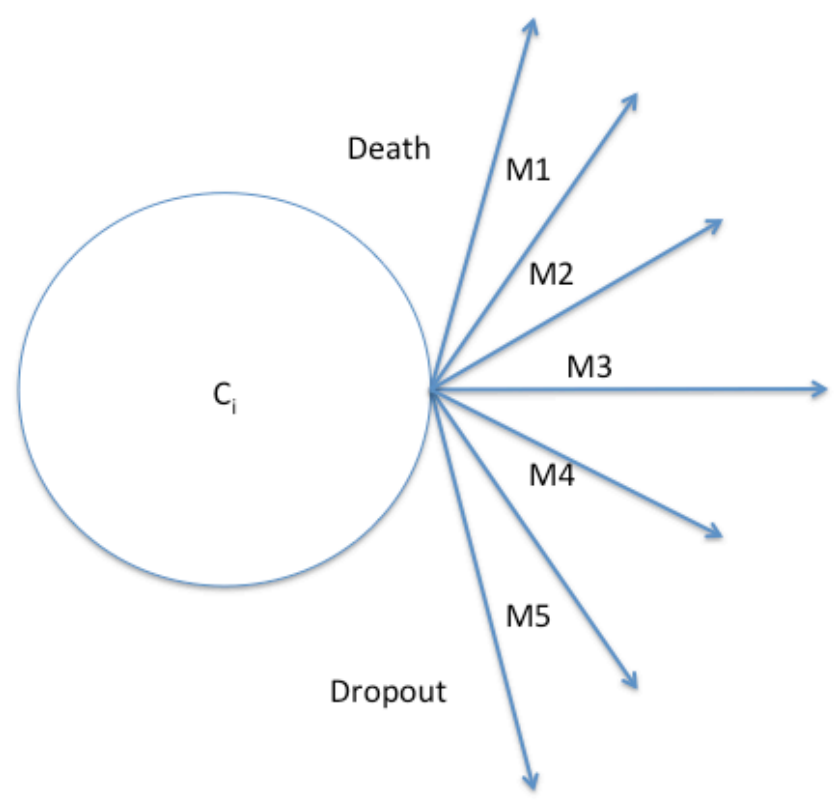

Figure 7: D-MODT Chance Nodes

1. Death: The patient has died

2. M1: The patient's MELD score has transitioned to $[0,10)$

3. M2: The patient's MELD score has transitioned to $[10,15)$

4. M3: The patient's MELD score has transitioned to $[15,20)$

5. M4: The patient's MELD score has transitioned to [20, 25)

6. M5: The patient's MELD score has transitioned to 25 or above

It is important to note that, in the above figure, the prior MELD score is assumed as an input, either from the patient's initial calculated MELD, or the scan performed at the last checkup. The mathematics governing these transitions are given in section 4.2.4. Moving forward from this chance node, all of the arcs (except death, at which the decision tree is terminated) transition to another decision node. If the patient has been categorized into one of the MELD states (i.e., M1-M5), then he is still eligible for OLT 
and moves to another decision node as in Figure 6. If he drops out from the wait list, his next and final decision node is the post-dropout decision node, which is depicted along with its two therapeutic options below in Figure 8:

\section{Pre-Transplant D-MODT: Post-Dropout Decision Node}

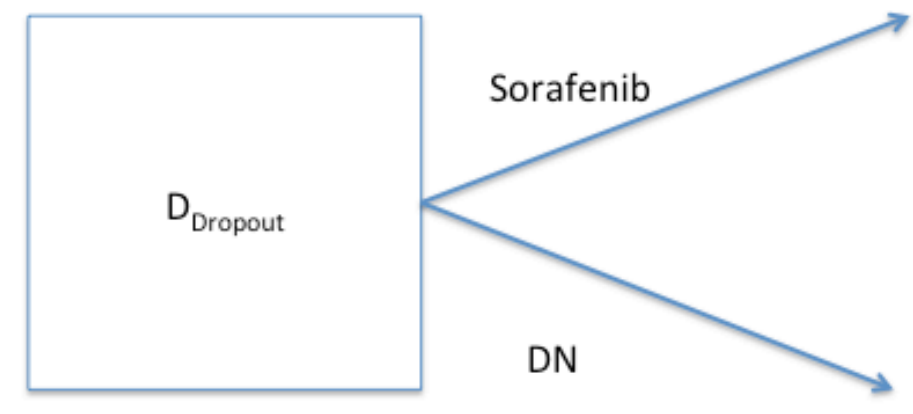

Figure 8: Post-Dropout Decision Node

1. Sorafenib: the patient is administered sorafenib for the remainder of his treatment schedule

2. DN: "Do nothing" i.e., no therapy

These three nodes comprise the pre-transplant decision tree. At the patient's expected time of transplantation (i.e., median wait time from Appendix A) according to his initial assigned MELD score, the following decision node occurs (if the patient is has not yet died or dropped out from the wait list): 


\section{D-MODT: Transplant Node}

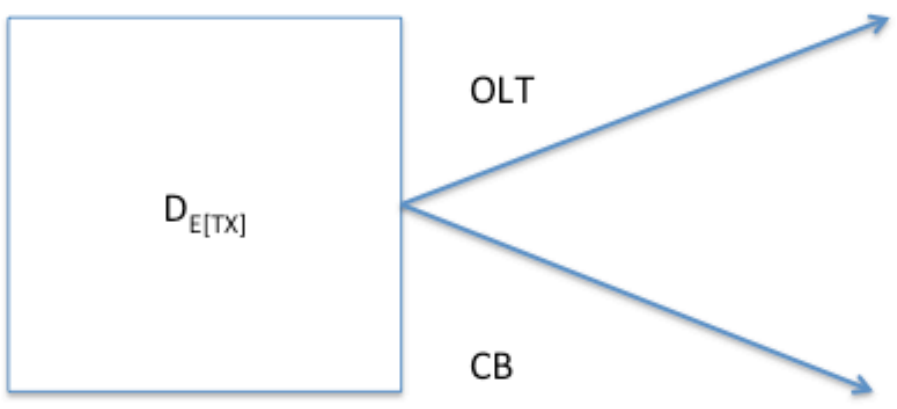

Figure 9: Transplant Decision Node

1. OLT: Liver transplant is performed

2. CB: No transplant is performed, patient continues his bridging therapy

\subsubsection{Complete D-MODT Structure}

In Figure 10, the sequence of decision and chance nodes is illustrated in a complete D-MODT for HCC treatments. Because each decision tree is of custom length for the patient in question, the pre-transplant tree extends to a hypothetical E[TX], which represents his median time until transplant based off of his region and initial MELD score. Note that, for readability's sake, only one chance node and decision node are depicted at each month, although from the above methodology it should be understood that every decision arc leads to another chance node, etc. A “...” after a node indicates that paths from it, the same as those depicted in nodes of the same month, spawn from it 
and continue on for the remainder of the decision tree. The objectives under consideration are lifetime provided and cost associated with treatment.

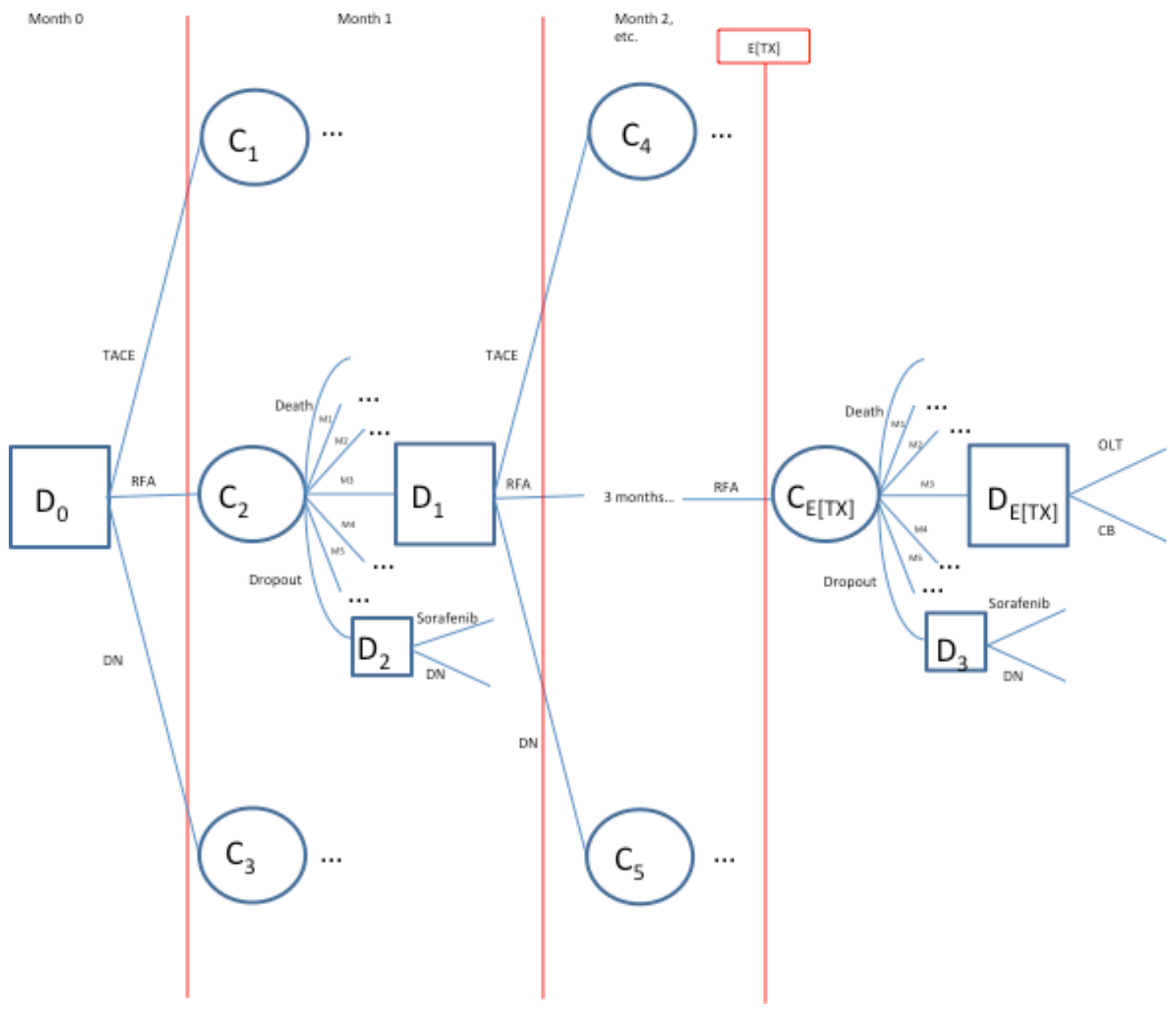

Figure 10: Full D-MODT for HCC Treatments

\subsubsection{Mathematical Solution Procedure}

Corresponding to the MODT solution procedure outlined in Haimes (2011), the D-MODT for HCC is solved via the process of averaging out and folding back. Every chance node is solved by "averaging out," where the probability associated with each arc 
is multiplied by that arc's damage vector via vector multiplication. Take, for example, the following chance node depicted in Figure 11:

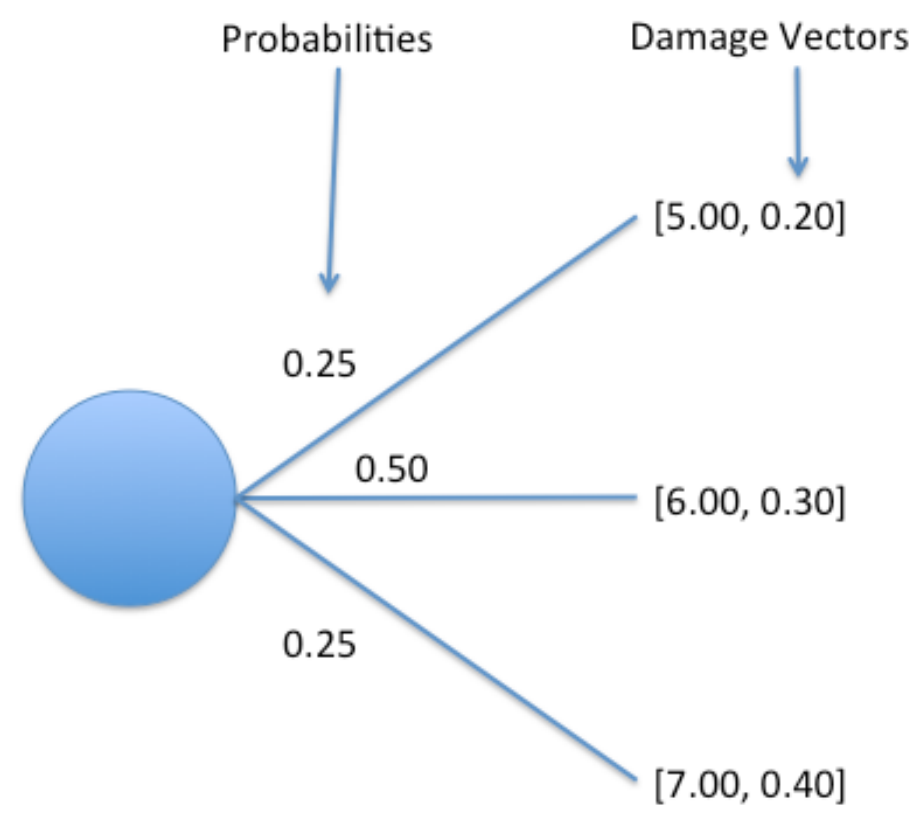

Figure 11: Sample Chance Node

This node would be solved via the calculations in Eqn. (8):

$$
\left[\begin{array}{l}
0.25 * 5.00+0.50 * 6.00+0.25 * 7.00 \\
0.25 * 0.20+0.50 * 0.30+0.25 * 0.40
\end{array}\right]=\left[\begin{array}{l}
6.00 \\
0.30
\end{array}\right]
$$

In the case of the D-MODT for HCC, this would equate to multiplying the damage vector (Life and Cost) associated with each of \{Death, M1-M5, Dropout $\}$ with their respective probabilities of occurrence at each chance node $C_{i}$ :

$$
\left[\begin{array}{l}
L_{C i} \\
C_{C i}
\end{array}\right]=\left[\begin{array}{l}
P(\text { Death }) * E\left[L_{\text {Death }}\right]+P(M 1) * E\left[L_{M 1}\right]+\cdots+P(\text { Drop }) * E\left[L_{\text {Drop }}\right] \\
P(\text { Death }) * E\left[C_{\text {Death }}\right]+P(M 1) * E\left[C_{M 1}\right]+\cdots+P(\text { Drop }) * E\left[C_{\text {Drop }}\right]
\end{array}\right]
$$


Where $L$ represents the lifetime provided and $C$ represents the cost associated with a particular arc. The $\mathrm{P}(\mathrm{M} 1)$, etc., represent the probability of a transition to the M1 MELD bucket. Of course, the probability of this transition is dependent on many factors, such as the patient's prior MELD score, as well as the treatment administered at the previous month. Let us define $i>j$ as indices in the set of months $[0,1, \ldots n\}$, where $n$ is the final checkup (i.e., the median time until transplant, where the pre-transplant decision tree ends) and the set of treatments $T(i)=\{T A C E, R F A, D N\}$ as the treatment administered at month $i$. Also, define $M(i)$ as a patient's MELD bucket [1,5] at month $i$. Now, we can calculate the transitions between MELD buckets via Bayes' Theorem:

$$
P(M(i) \mid M(j), T(j))=\frac{P(M(j), T(j) \mid M(i)) * P(M(i))}{\sum_{i} P(M(j), T(j) \mid M(i)) * P(M(i))}
$$

Where the denominator on the left hand side of the equation comes from the partitioning of the MELD state space into five buckets.

By definition, the probabilities associated with all arcs emanating from a chance node should sum to 1. The calculation in Eqn. (9) solves an individual chance node. Once all chance nodes corresponding to arcs emanating from a particular decision node are solved in the same manner as Eqn. (9), their solutions are compared in terms of Pareto-optimality, and the inferior solutions are discarded from the remainder of the tree. This procedure is repeated until the starting point of the tree, the initial decision of bridging therapy at Month 0 , is reached. 


\subsection{Advantages of D-MODT Methodology}

The D-MODT methodology for HCC treatments has many advantages over both non-Bayesian (e.g., Markov) models, as well as earlier versions of the MODT for HCC (Bleistein, 2011). The following paragraphs summarize these advantages, and are structured as a response to the motivating questions posed in section 4.1.

Time dependency and conditional probabilities. As noted multiple times, the time domain is central to HCC treatment. Physicians must not only prescribe the best therapies to their patients, but at the proper times in order to preserve their state of health as they await OLT. Markov models account for time in the sense that their transitions are viewed as homogeneous steps throughout time, but they lack the refinement that is present in the D-MODT's ability to move through the nonhomogeneous lattice of checkups that characterizes HCC treatment. All probabilities in the model can be conditioned on their time of occurrence, given the availability of appropriate data. In addition, the Bayesian approach employed in D-MODT takes into account all past events in the patient's therapy, as opposed to the Markov property's ignorance of all but the most recent event. This mathematical approach gives D-MODT the ability to distinguish, at step $i$, between patients who have had different treatments past the $i-1^{\text {st }}$ step, which is one of the many ways D-MODT is much more patient-specific.

Patient specificity. In many Markovian approaches, a homogeneous patient population is assumed. This is far from realistic, especially given HCC's prevalence as the fifth-most common type of cancer. D-MODT makes use of the calculated MELD score as a state variable, as it inputs a patient's initial MELD score at the Month 0 
decision node $\left(\mathrm{D}_{0}\right)$. This initial MELD score is then used to calculate probabilities of transition to future chance nodes' MELD buckets. For example, the probabilities associated with a patient's D-MODT whose initial calculated MELD is 12 will be very different from those of a patient whose initial MELD is 24, since the latter patient will have a higher probability of death or dropout at the first chance node. Thus, every patient will have an individual D-MODT based off of his initial calculated MELD score as well as his geographic location (see below).

Geographic specificity. The wait times for transplantation vary greatly with one's geographic location, and thus also do one's chances of death or dropout while on the waiting list. A good model should take into account one's location and forecast the length of bridging therapy accordingly. The D-MODT takes as an input one's OPTN region (1-11) and utilizes this figure (see Appendix A for median wait times by region and initial assigned MELD) to structure the pre-transplant decision tree's horizontal length, which corresponds to the time spent waiting for transplantation.

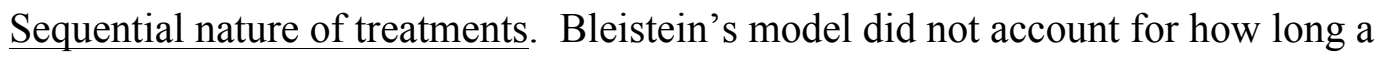
treatment was administered, nor was it built to accommodate treatment sequences where different treatments are administered at different times. D-MODT addresses this by charting out all possible combinations of therapies given the one and three month checkup interval assumption. Once the model can be fully populated, this approach will be a powerful tool for researchers seeking to determine at what points the administration of certain therapies are most efficacious to a patient.

Additional chance node granularity. Bleistein's model only allowed for three possibilities at his single pre-transplant probabilistic chance node: death, dropout, and 
eligibility for transplantation. D-MODT still accounts for the fact that patients may die and dropout before they receive a transplant, but is much more granular with respect to those patients who remain eligible for transplant. Many patients on the wait list will eventually receive a transplant, and there must be some distinction made among them in terms of their status while they await transplant, so that appropriate bridging therapies may in the meantime be assigned to them. D-MODT provides additional granularity by defining a patient's calculated MELD score as the state variable, and partitions the spectrum of MELD scores into five "buckets," among which patients transition throughout their treatment. Given a patient's progression through these buckets, the DMODT will assign appropriate probabilities so that the physician may, at every update of the patient's status, analyze his most recent expected life and cost associated with each therapeutic option.

Hierarchical structure of nodes. The structure of D-MODT (and MODT) is that of a hierarchical tree-of-trees. Each decision node can be viewed as the root of its own tree and, via the same mathematical procedure that is used to solve the model in its entirety, can be solved to determine the set of Pareto-optimal treatment sequences stemming from that specific node. In that sense, the D-MODT is far more than a tool used to project efficacious sequences for a patient's entire treatment process; rather, it is a guide with which a decision-maker can study the effects of employing any therapy at any time throughout a hypothetical schedule of treatments. The tree can likewise be decomposed to elucidate answer to one's specific questions that need not go through the entire model's solution process to answer. 
This hierarchical nature allows the D-MODT to be employed as both a short and long-term forecasting tool. A treatment administered at a checkup seeks to give the patient the best chance of survival until the next checkup. Just as a lengthy D-MODT can take as an input a patient's initial calculated MELD score, a physician can also input a patient's current MELD score and solve a one-period D-MODT (i.e. one or three months, depending on the patient's last therapy) to gather information on the tradeoffs among the therapies he is currently considering prescribing.

Model mutability. Although the D-MODT in this paper focuses on three therapies and two objectives, the model can easily be tailored to answer more general questions by adding branches to decision nodes (if one wishes to consider more therapeutic options), or adding dimensions to the damage vectors (if one wishes to consider more objectives). This, of course, is dependent upon the availability of data with which to construct further decision trees.

It has been stressed that D-MODT is a methodology that is unique to each patient. Thus, the population of a general model a la Bleistein (2011) and the many Markovian papers in the literature is impossible. To demonstrate the D-MODT solution procedure in detail, the following case study is provided. A general discussion of D-MODT, future directions, identification of areas for improvement, and conclusion is to follow. 


\section{D-MODT FOR HCC: AN EXPLANATIVE CASE STUDY}

The following sections are meant to serve as a guide to solving a D-MODT for $\mathrm{HCC}$, as well as review the database and provide information on data acquisition for those interested. The case study is not meant as an argument for or against any therapeutic sequence, and truthfully could not be used as one due to the many assumptions taken in populating it with data. This section also demonstrates how one disadvantage of the D-MODT methodology lies in its massive data requirements, and should serve as motivation for future modeling efforts that still maintain its level of sophistication while easing its current data requirements. A summary of the overall thesis, future directions, and conclusion are included at the end of this section.

\subsection{Simplified Model for Case Study}

A good case study should demonstrate the efficacy of a methodology without a high level of complexity or unnecessary study on the part of the reader. Thus, much thought was devoted to constructing a numerical example for this thesis that would highlight the benefits of its methodology while still remaining relatively simple and easy to understand. Recall that the D-MODT can be viewed hierarchically as a "tree of trees"- that is, each decision node is essentially the root of its own tree. Thus, the solution procedure explained below can be repeated for additional nodes if one wishes to solve larger D-MODTs. To demonstrate this paper's methodology, one month's worth of 
decisions were simulated. This decision was made for simplicity's sake; several months' worth of models were charted out. A three-month D-MODT contained 3,486 total nodes, the two-month contained 337 nodes, and the one-month contained 22 nodes. The length of the one-month tree corresponds to a one-month wait time, as (according to Appendix A) is the case for UNOS Regions 6 (WY, CO, NE, KS, IA, MO) and 10 (MI, IN, OH). Yet, this tree can also be viewed hierarchically as one month in a sequence of many. The one-month D-MODT is charted below in Figure 12.

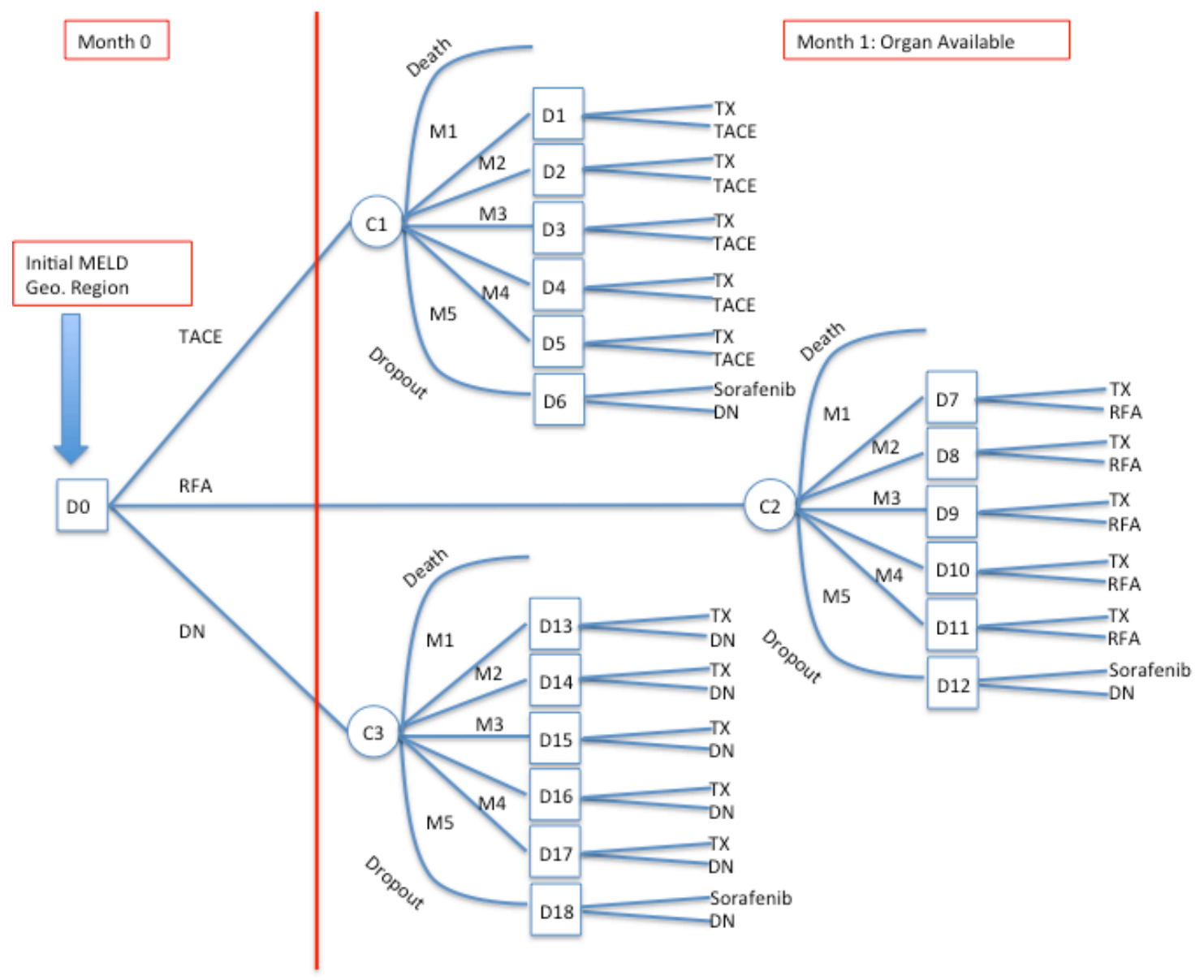

Figure 12: D-MODT for One Month

In Figure 12, an initial therapy decision $\{\mathrm{TACE}, \mathrm{RFA}, \mathrm{DN}\}$ is made at Month 0 . In the time between Month 0 and Month 1, the patient ether dies, remains eligible for transplant 
(i.e., transitions among the MELD buckets), or drops out from the transplant waiting list.

If the patient remains eligible, the decision is immediately made whether or not to undergo OLT.

Hierarchically, Figure 12 can be viewed as one of the decision nodes D1-D5 in Figure 13, which depicts a two-month D-MODT.

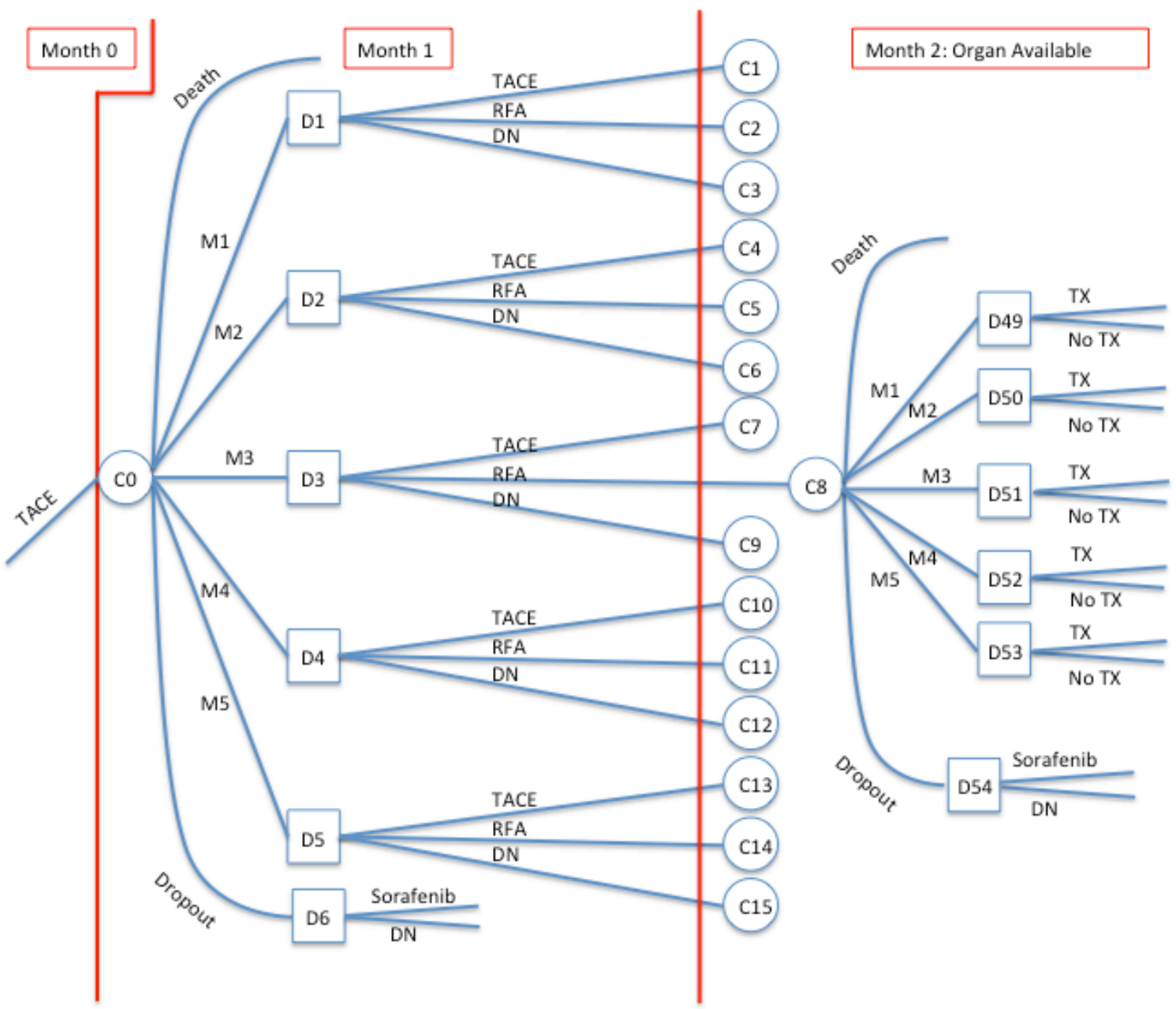

Figure 13: 2-Month D-MODT for HCC 
Note that, in a larger (i.e., spanning more months) D-MODT, the branches emanating from nodes D1-D5 would not all span one month, as they do in Figure 13. Rather, they would follow the one-and-three month assumption. For example, in a larger tree, any of the TACE branches emanating from nodes D1-D5 would span three months, to month 4, as the model assumes that two consecutive treatments of the same time will cause the time between checkups to be three months. However, since the expected time until transplant is only 2 months in the D-MODT in Figure 13, the patient's state of health will be observed instead after one month, since at this point he will immediately be transplanted as the donor liver is available.

The following sections describe the assumptions behind and the population of the one-month D-MODT pictured in Figure 12.

\subsection{Assumptions}

The following assumptions were made for the model in this case study:

1) The set of treatment options included $\{$ TACE, RFA, DN $\}$ as bridges while on the waitlist to OLT. Sorafenib becomes an option once the patient dropped out from the wait list.

2) At each chance node, the patient's state is observed, which corresponds to a checkup. These feature the absorbing states of death or dropout, as well as the transient states of the five MELD buckets.

3) The set of patients considered were those receiving a priority MELD score for a stage T2 HCC diagnosis. 
4) If the patient was still on the waitlist at his median time to transplant (1 month according to Appendix A), a decision would be made immediately regarding whether or not to perform the transplantation.

5) Probabilities with which the tree was populated, as well as the final damage vectors, were derived from the Standard Transplant Analysis and Research (STAR) database, and the relevant literature.

\subsection{Review of the Database and Model Population}

\subsubsection{STAR Database}

This section reviews the various methods used to populate the model used in this paper's case study. As much as was possible, data was collected from the UNOS Standard Transplant Analysis and Research (STAR) database. This database was made available upon request by UNOS and contains data files for HCC patients who were placed on the waitlist for OLT between December 11, 1985 and April 30, 2012. There are two datasets in this database which were of interest to this study: the LIVER_PUBLIC_USE dataset, which contains general information on patients such as date of registration, calculated MELD scores, and a multitude of demographic statistics; the HCC_TUMOR_DATA dataset contains information such as if RFA or TACE was performed on the patient's lesion, the type of tumor (T1 or T2), and case outcomes. The two datasets are linked to each other via the WL_ID_CODE identifier. An index of variables examined in this study from both datasets are provided in Appendix B. 
The first step to harvesting useful probabilities from this data was to whittle down the massive datasets into only the patients this study wished to consider. The strategy employed was to reduce the two sizes of the two datasets independently to capture patients within those datasets who met the assumptions for this study, and then to match those datasets by WL_ID_CODE and use as a final dataset only those patients who were still in both datasets. Recall that one assumption was that patients had to be diagnosed with T2 HCC. The HCC_TUMOR_DATA dataset was filtered so that it only contained patients whose first chronological ORIG_APP_DATE (original application date) entry had "HCC Meeting Policy 3.6.4.4 Criteria (Stage T2)" in the HCC_DIAG (HCC diagnosis) field. This reduced the original set of 19,087 patients to 15,155 within this dataset.

The next step was to consider only the patients in the LIVER_PUBLIC_USE dataset that did not receive an LDLT. The DON_TY (donor type) field indicates whether the donor was deceased or living. Records with an "L" entry in this field (living) were discarded. Additionally, the EXC_HCC field indicates whether or not a patient was given a wait list exception (i.e., MELD priority) due to HCC. Also, any patients who had been previously transplanted (value $>0$ in the NUM_PREV_TX field) were removed. Filtering the dataset for these criteria reduced the dataset from 209,969 patients to 14,013 patients.

The two filtered datasets were then combined into one dataset containing only those patients whose WL_ID_CODEs that appeared in both datasets. This dataset contained 20,875 records for 11,320 patients. It is important to note that each patient 
had, on average, less than two entries, which makes difficult the calculations of entry-toentry probabilities, such as those that must be conditioned on certain months.

Next, the data from this main dataset was filtered to examine patients who received TACE, RFA, DN as their pre-transplant bridge. The indicators in the CHEMOEMBO, and RFA fields were employed to select patients who only underwent these two therapies. For the DN set of patients, the set of patients who did not receive any (CHEMOEMBO, CHEM_ABLAT, CRYO, RESECT, RFA) bridges were considered. Patients who received combinations of bridges were discarded. Together, the set of patients numbered 12,370. Table 1 lists the number of patients in each of these categories:

\begin{tabular}{|l|l|}
\hline Therapy & Number of Patients \\
\hline TACE & 4267 \\
\hline RFA & 1401 \\
\hline DN & 6702 \\
\hline
\end{tabular}

Table 1: Bridge Statistics from STAR Database

The next step in populating the model was to divide each of the three bridging datasets into three groups of patients: those who died within a month, those who were eligible for transplant, and those who dropped out from the waiting list within a month. The fields DAYSWAIT_CHRON, END_DATE, INIT_DATE, PSTATUS, REM_CD, and TX_DATE were used to calculate these statistics. This information is displayed below in Table 2 and can be used as prior probabilities for Bayesian calculations within the therapy groups: 


\begin{tabular}{|l|l|l|l|}
\hline Therapy & \% Died (1M) & \% Dropped Out (1M) & \% Eligible \\
\hline TACE & 3.37 & 1.24 & 95.39 \\
\hline RFA & 4.14 & 0.86 & 95.00 \\
\hline DN & 5.09 & 0.81 & 94.10 \\
\hline
\end{tabular}

Table 2: Overall Statistics for Bridging Therapies (STAR Database)

These probabilities must be conditioned on the prior stage's MELD score (in this case study, this corresponds to the patient's initial MELD). Due to the lack of multiple entries for many patients, the transitions between MELD scores are calculated by examining the difference between the initial (INIT_MELD or MELD_PELD_LAB_SCORE) and the final (FINAL_MELD) scores. Within each therapy, the probabilities associated with the arcs emanating from that therapy's Month 1 chance node were calculated. In the database, patients who had died or dropped out from the waiting list often lacked MELD information. To account for this, the conditioned probabilities of death and dropout on any MELD score are assumed to be the unconditioned probabilities of death or dropout (regardless of initial MELD). Table 3 depicts the probabilities associated with the chance arcs, given that the patient's initial MELD score was below 10. Appendix C lists the rests of the tables (for other initial MELD scores).

\begin{tabular}{|l|l|l|l|l|l|l|l|}
\hline Therapy & P(De|M1) & P(M1|M1) & P(M2|M1) & P(M3|M1) & P(M4|M1) & P(M5|M1) & P(Dr|M1) \\
\hline TACE & 3.37 & 65.11 & 22.73 & 5.68 & 1.65 & 0.22 & 1.24 \\
\hline RFA & 4.14 & 67.64 & 21.33 & 4.31 & 1.29 & 0.43 & 0.86 \\
\hline DN & 5.09 & 61.87 & 25.33 & 4.93 & 1.60 & 0.37 & 0.81 \\
\hline
\end{tabular}

Table 3: Chance Node Probabilities For Initial Meld [0,10) 
Note that some probabilities, such as $\mathrm{P}(\mathrm{M} 5 \mid \mathrm{M} 1)$ are assigned $\mathrm{a}<0.00001$ probability since they did not occur in the database but are still technically feasible.

\subsubsection{Data from the Literature}

Since the STAR database did not contain adequate data regarding month-tomonth checkups, the literature was consulted for monthly survival statistics and costs for bridging therapies and transplantation. To remain consistent with Bleistein (2011), the costs per event of OLT, DN TACE, and RFA were taken from Naugler and Sonnenberg (2010). The cost of Sorafenib was taken from Bleistein (2011). It is important to note that the DN therapy still has a cost attached to it, which is due to clinical expenses. Table 4 displays this information.

\begin{tabular}{|c|c|c|}
\hline Therapy & Cost & Reference \\
\hline TACE & $\$ 6,080 /$ month & Naugler and Sonnenberg (2010) \\
\hline RFA & $\$ 2,300 /$ month & Naugler and Sonnenberg (2010) \\
\hline Sorafenib & $\$ 5,000$ (one-time) & Bleistein (2011) \\
\hline OLT & $\$ 278,800$ (one-time) & Naugler and Sonnenberg (2010) \\
\hline DN & $\$ 287 /$ month & Naugler and Sonnenberg (2010) \\
\hline
\end{tabular}

Table 4: Costs of Therapies

The above costs are incurred at each month that their therapy is employed. Additionally, data was taken from the literature regarding expected additional survival time. Table 5 displays this information. 


\begin{tabular}{|c|c|c|}
\hline Therapy & E[Additional Life] & Reference \\
\hline TACE & 51.23 months & Naugler and Sonnenberg (2010) \\
\hline RFA & 63.28 months & Naugler and Sonnenberg (2010) \\
\hline Sorafenib & 3.00 months & Multiple \\
\hline OLT & 81.00 months & Llovet, Fuster, and Bruix (1999) \\
\hline DN & 0.00 months & Assumed \\
\hline
\end{tabular}

Table 5: Expected Survival Times

These survival times correspond to the survival benefit of their therapies when administered independently of other therapies. It is assumed that DN provides no additional survival. In this case study, these are the expected survival times associated with the arcs emanating from the Month 1 decision nodes.

\subsubsection{Future Data Needs}

One of the benefits of harvesting data from a large dataset is the illumination of areas where data is currently not present. There are currently many unpopulated areas whose population would allow for better modeling efforts. One such area concerns combinations of therapies. The D-MODT methodology is built to allow for the physician to change a patient's bridging therapy at every checkup. To accurately populate the damage vectors corresponding to sequences where combinations of therapies are prescribed in a large-scale D-MODT, this data needs to exist. Current clinical studies such as those in this paper's literature review typically consider the effects of a single 
therapy over an amount of time. What is the efficacy of employing, say, TACE for 1 month and RFA for 3 months, followed again by TACE for 2 months? While the expected monthly survival associated each independent therapy is available in the literature, the survival associated with therapeutic combinations has yet to be determined (to the best of the author's knowledge).

The passage of more time will allow for the relevant medical databases to continue growing in number. A mere 10 years have passed since the implementation of MELD, and there simply is not yet enough data for the population of all points of a robust and lengthy model such as a large-scale D-MODT. The inclusion of more patients will allow "rarer" sections of models to be populated with relevant data, and will refine the existing data (such as MELD transition probabilities). In this study, the time between MELD measurements was not included as a factor due to the lack of such data. Should a D-MODT be charted for 12 months, there would be very few data points with which to populate the arcs in say, months 10-12 of the tree, since the average number of checkups recorded for a patient in the STAR database is less than 2.

Additionally, studies that provide more granularity with regards to the impacts of a therapy upon a patient with a certain MELD score will help populate models such as the D-MODT that use MELD as the patient's state of health and condition its probabilities upon it. How does the impact of a therapy (with respect to lifetime provided and cost incur) change when it is administered to patients who have transitioned to particular MELD scores at a certain time? 


\subsection{Case Study Results}

After the model was populated with data via the techniques in the preceding section, the one-month D-MODT depicted in Figure 12 was solved according to the solution procedure outlined in section 4.2.4. Recall that the D-MODT has two inputs: the patient's initial calculated MELD score, and his geographic region. In this case study, the assumption was already made that the patient's geographic region was 6 or 10, which coincides with a D-MODT of one month's length. Regarding the patient's initial MELD score, decision trees were constructed for the five possible initial MELD buckets. The decision tree to be discussed in detail in this text will be that of a patient whose initial calculated MELD score lies within the first bucket: $[0,10)$. Appendix D contains the decision trees corresponding to the four other MELD buckets.

To ascertain the values of the damage vectors [L,C] associated with each decision arc emanating from the Month 1 decision nodes, the expected survival and cost associated with each therapy were added to the one-month cost and additional survival of the bridging therapy employed at Month 0 . Once this expected survival and cost was computed for every Month 1 decision arc, these vectors were compared in terms of Pareto-optimality. Recall that this case study wishes to maximize the expected additional lifetime provided by a treatment, while minimizing its cost. Thus, "folding back" is employed here, and dominated vectors are discarded. All of the Month 1 vectors were Pareto-optimal with respect to each other (i.e., versus the other vectors at a particular decision node). These values are depicted in Table 6. This table of damage vectors is 
used for the decision tree where the patient's initial MELD score lies in the first MELD bucket.

Next, the Month 1 chance nodes must be averaged out. Every Pareto-optimal decision arc for Month 1 (pictured in Table 6) is multiplied by its probability. Since there were two non-dominated decision for each of the 6 branches emanating from every chance node, there are $3 \times 2^{6}=192$ sequences to consider. 


\begin{tabular}{|c|c|c|c|}
\hline Node & Decision & $\mathbf{E}[\mathbf{L}]$ & $\mathrm{E}[\mathrm{C}]$ \\
\hline \multirow[t]{2}{*}{1} & $\mathrm{TX}$ & 81.43 & 284080.00 \\
\hline & TACE & 51.66 & 30384.00 \\
\hline \multirow[t]{2}{*}{2} & $\mathrm{TX}$ & 81.43 & 284080.00 \\
\hline & TACE & 51.66 & 30384.00 \\
\hline \multirow[t]{2}{*}{3} & $\mathrm{TX}$ & 81.43 & 284080.00 \\
\hline & TACE & 51.66 & 30384.00 \\
\hline \multirow[t]{2}{*}{4} & $\mathrm{TX}$ & 81.43 & 284080.00 \\
\hline & TACE & 51.66 & 30384.00 \\
\hline \multirow[t]{2}{*}{5} & $\mathrm{TX}$ & 81.43 & 284080.00 \\
\hline & TACE & 51.66 & 30384.00 \\
\hline \multirow[t]{2}{*}{6} & Sorafenib & 3.43 & 11080.00 \\
\hline & DN & 0.43 & 6366.67 \\
\hline \multirow[t]{2}{*}{7} & $\mathrm{TX}$ & 81.53 & 280300.00 \\
\hline & RFA & 63.81 & 20686.00 \\
\hline \multirow[t]{2}{*}{8} & $\mathrm{TX}$ & 81.53 & 280300.00 \\
\hline & RFA & 63.81 & 20686.00 \\
\hline \multirow[t]{2}{*}{9} & $\mathrm{TX}$ & 81.53 & 280300.00 \\
\hline & RFA & 63.81 & 20686.00 \\
\hline \multirow[t]{2}{*}{10} & $\mathrm{TX}$ & 81.53 & 280300.00 \\
\hline & RFA & 63.81 & 20686.00 \\
\hline \multirow[t]{2}{*}{11} & $\mathrm{TX}$ & 81.53 & 280300.00 \\
\hline & RFA & 63.81 & 20686.00 \\
\hline \multirow[t]{2}{*}{12} & Sorafenib & 3.53 & 7300.00 \\
\hline & DN & 0.53 & 2586.67 \\
\hline \multirow[t]{2}{*}{13} & $\mathrm{TX}$ & 81.00 & 278286.67 \\
\hline & $\mathrm{DN}$ & 0.00 & 1146.67 \\
\hline \multirow[t]{2}{*}{14} & $\mathrm{TX}$ & 81.00 & 278286.67 \\
\hline & $\mathrm{DN}$ & 0.00 & 1146.67 \\
\hline \multirow[t]{2}{*}{15} & $\mathrm{TX}$ & 81.00 & 278286.67 \\
\hline & $\mathrm{DN}$ & 0.00 & 1146.67 \\
\hline \multirow[t]{2}{*}{16} & $\mathrm{TX}$ & 81.00 & 278286.67 \\
\hline & $\mathrm{DN}$ & 0.00 & 1146.67 \\
\hline \multirow[t]{2}{*}{17} & $\mathrm{TX}$ & 81.00 & 5286.67 \\
\hline & $\mathrm{DN}$ & 0.00 & 1146.67 \\
\hline \multirow[t]{2}{*}{18} & Sorafenib & 3.00 & 5286.67 \\
\hline & $\mathrm{DN}$ & 0.00 & 1146.67 \\
\hline
\end{tabular}

\section{TABLE 6: Damage Vectors for Month 1 Decision Nodes}

For example, consider the combination of decision options at the TACE arc emanating from decision node D0. One of these decision rules (the first row in Table 8), states that 
a transplantation will be performed regardless of the MELD bucket the patient transitions into, and that sorafenib will be provided if the patient drops out from the waiting list.

That is, this combination is (TX|M1, TX|M2, TX|M3, TX|M4, TX|M5,

Sorafenib|Dropout). The damage vector for this decision rule is calculated

mathematically as:

$$
\begin{aligned}
& {\left[\begin{array}{l}
L_{T X} * P(M 1)+L_{T X} * P(M 2)+L_{T X} * P(M 3)+L_{T X} * P(M 4)+L_{T X} * P(M 5)+L_{\text {Soraf. }} * P(\text { Drop. } \\
C_{T X} * P(M 1)+C_{T X} * P(M 2)+C_{T X} * P(M 3)+C_{T X} * P(M 4)+C_{T X} * P(M 5)+C_{\text {Soraf. }} * P(\text { Drop. }
\end{array}\right.} \\
& =\left[\begin{array}{c}
81.43(0.65+0.23+0.06+0.02+0.002)+3.43 * 0.01 \\
284080(0.65+0.23+0.06+0.02+0.002)+11080 * 0.01
\end{array}\right] \cong\left[\begin{array}{c}
77.72 \\
271121.30
\end{array}\right](11)
\end{aligned}
$$

where the elements of the vector correspond to this policy offering an additional expected life of 77.72 months at an expected cost of $\$ 271,121.30$. This procedure is repeated for each of the 192 possible policies. These tables are very large, and are located in Appendix D for the interested reader. Once these calculations are complete, the policies are compared in terms of Pareto-optimality both in terms of the set of policies emanating from each of the three D0 decision arcs, as well as overall (i.e., against all 191 other policies). For example, the policy (Policy 128 in Appendix D) that suggests (RFA|M1, RFA|M2, RFA|M3, RFA|M4, RFA|M5, DN|Dropout) after initial RFA bridging dominates the policy (Policy 33 in Appendix D) that suggests (TACE|M1, TX|M2, TX|M3, TX|M4, TX|M5, Sorafenib|Dropout) after initial DN bridging because:

$$
\left[\begin{array}{c}
60.62 \\
19673.95
\end{array}\right]_{128}>\left[\begin{array}{c}
58.33 \\
105939.84
\end{array}\right]_{33}
$$


Note that the "greater" sign in Equation 12 is expressed in the sense of dominance of objective functions, as opposed to in the traditional mathematical sense. Every policy is compared to the other policies as in Equation 12. After discarding every inferior policy, the decision-maker is left with a set of Pareto-optimal treatment sequences. Below, Table 7 displays the number of these policies for each of the five D-MODTs corresponding to the five initial calculated MELD buckets. To view the list of these policies, one many consult Appendix D, which contains a "P-O" column (Pareto-optimal) for each of the five decision trees. Rows marked with an " $\mathrm{X}$ " in this column are inferior policies.

\begin{tabular}{|l|l|l|}
\hline Initial Calculated MELD & Optimal Policies & Inferior Policies \\
\hline$<10$ & 84 & 108 \\
\hline$[10,15)$ & 71 & 121 \\
\hline$[15,20)$ & 71 & 121 \\
\hline$[20,25)$ & 34 & 158 \\
\hline$\geq 25$ & 88 & 104 \\
\hline
\end{tabular}

Table 7: Number of Optimal/Inferior Policies for Each D-MODT

Note that the number of optimal policies decreases as the patient's initial calculated MELD score increases. The large number of optimal policies associated with the fifth MELD bucket (those scores greater than or equal to 25) should be viewed with caution, since there were by far less patients with initial MELD scores in this category: in the DN 
set of patients, for example, only 17 of the $4730(0.36 \%)$ had initial MELD scores at or over 25 .

For the model discussed in this case study (MELD $<10)$, the initial bridging therapies can be decomposed as follows:

\begin{tabular}{|c|c|c|c|c|}
\hline D0 Bridge & Optimal (of 64) & Inferior (of 64) & E[L] of Optimal & E[C] of Optimal \\
\hline TACE & 8 & 56 & 73.19 & 232682.51 \\
\hline RFA & 64 & 0 & 69.05 & 143010.86 \\
\hline DN & 12 & 52 & 2.85 & 10158.32 \\
\hline
\end{tabular}

Table 8: Statistics for Optimal Policies

Next, Pareto-optimality graphs were charted for each of the decision trees. For each decision tree, two charts were made: that of all the treatment strategies (inferior policies included), and that of only the Pareto-optimal strategies (the numbers of which are located in Table 8). Below are these two such graphs for the decision tree discussed in this case study, that of the initial calculated MELD $<10$. 


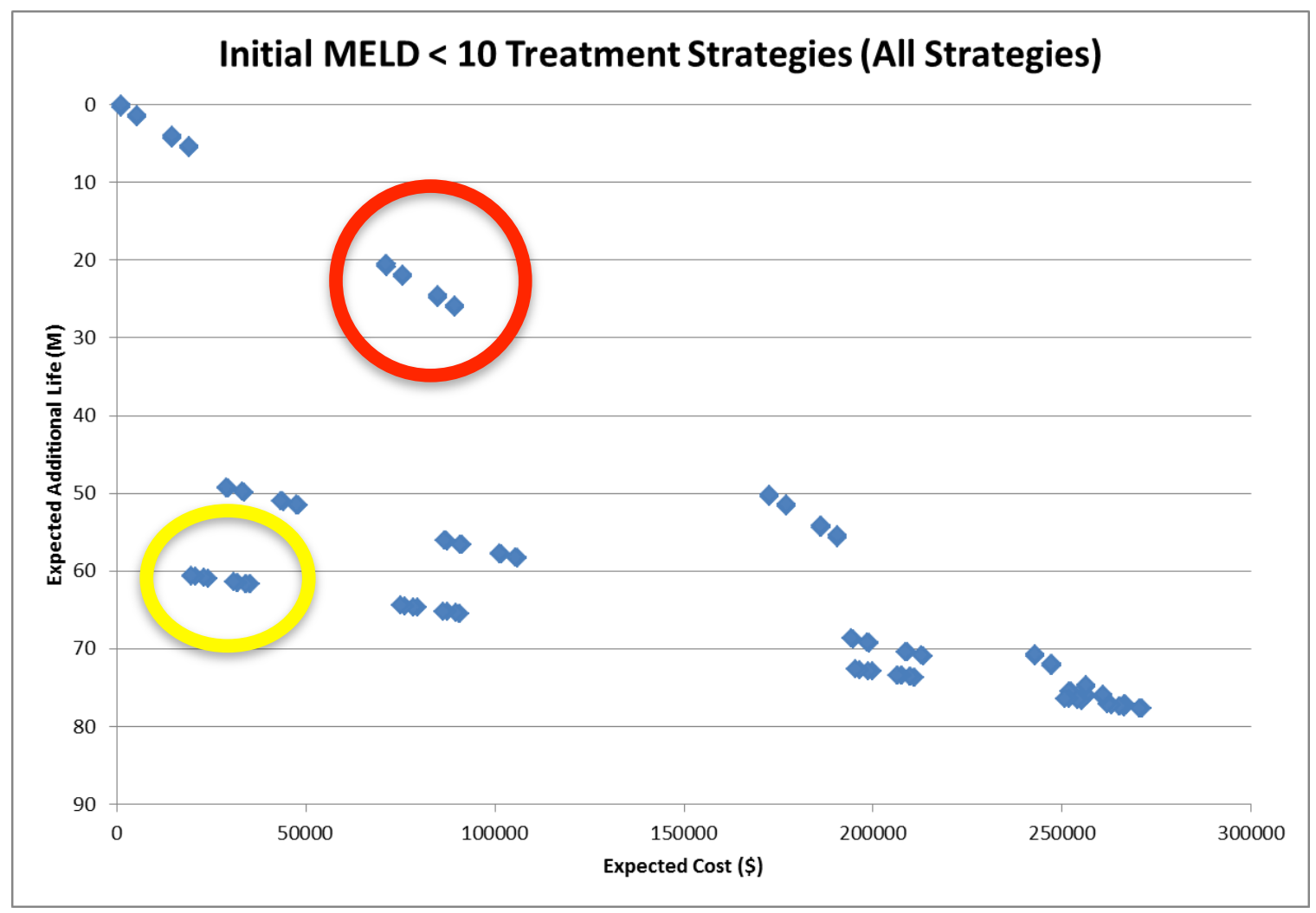

Figure 14: Plot of All Treatment Policies

Plotting the policies is another way to quickly identify which among them are dominated. In Figure 14 the objective functions define the graph's axes. Noting that the vertical axis (corresponding to the patient's expected additional life) has been inverted, Pareto-optimal solutions will be those that lie closest to the origin - that is, those that maximize the patient's expected additional life while minimizing his expected cost. Policies, such as those in the red circle in Figure 14, are dominated by policies such as those in the yellow circle. The points in the yellow circle fall closer to the origin with respect to both axes than those in the red circle, and thus are Pareto-optimal to those policies. 
Considering only those points that are deemed Pareto-optimal, Figure 15 was charted.

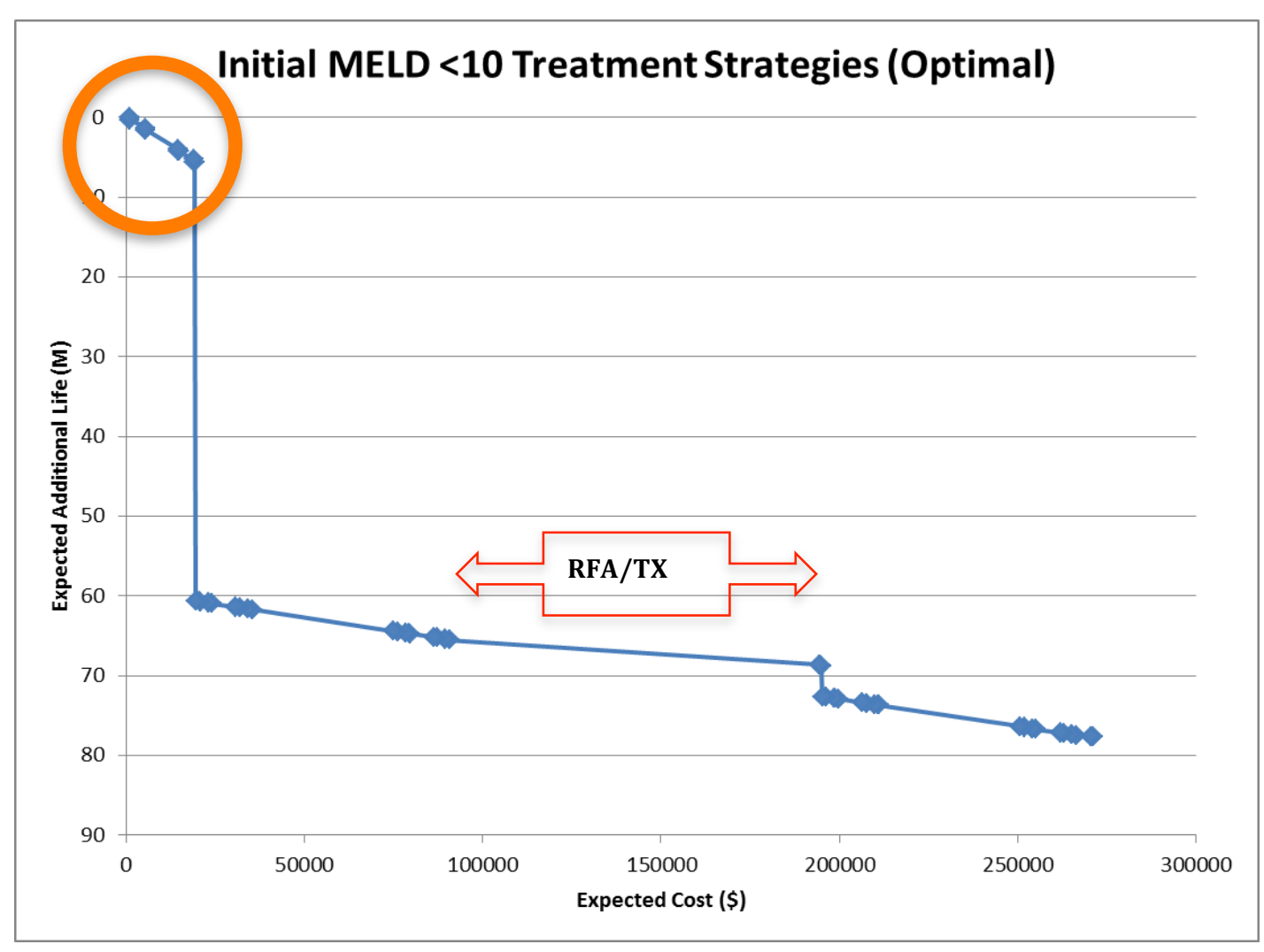

Figure 15: Optimal Treatment Policies

Note that none of the points in Figure 15 is closer to the origin with respect to both axes than any other point. Consulting a chart like this, a physician can now decide whether he values cost or life more in a patient's specific disease management scenario, and select a policy accordingly. The values of these points can be matched to their treatment policies via consulting Appendix D. For example, the points contained in the orange circle in Figure 15 are points featuring DN as the initial bridging therapy. These policies provide the patient with less than 10 months of expected additional life, and all have associated costs of less than $\$ 20,000$. Additionally, notice the separation between the two groups of points providing $60+$ months of additional expected life, noted by the red box and arrows. 
The large division between these two groups of points comes from the decision at node D7. At this node, RFA has already been administered as the patient's initial bridge, and his MELD score has transitioned from M1 to M1 (i.e., it has remained in the first $<10$ MELD bucket). The large divide between the groups of points comes from the decision to TX (points on the right) or to provide RFA (points on the left) at the time of organ availability. This is an excellent example of the benefits of D-MODT methodology; because so many $(68 \%)$ of patients whose initial MELD score transition into the M1 bucket at Month 1, the therapeutic decision at node D7 is crucial to the patient's expected life and cost. In a model where patient specificity and time are ignored, the criticality of this decision node may not be evident. Note that many of the points from both groups feature the decision to transplant, but it is for which group of patients that this decision is made that greatly impacts the expected value of the additional life and cost.

For the interested reader, Appendix E contains the graphs for all policies and Pareto-optimal policies for the remaining four initial MELD bucket models.

The above case study serves to provide the reader with an idea of how physicians may use this paper's methodological approach to project the efficacious impacts of competing therapies for both the present time (i.e., a single checkup; by folding back on the present decision) and for the entirety of a patient's treatment (i.e., by charting out an appropriately long decision tree). It is important to note that the D-MODT methodology is not confined strictly to the assumptions under which it was placed in this section. Although this case study only considered three bridging therapies, the inclusion of other therapies is not restricted. Additionally, a decision tree of any length can theoretically be constructed, so long as the data is present with which to populate it. Thus, this case study 
served as an example of how the D-MODT methodology may be applied in a particular instance. Even with a limited database and a short decision tree of one month, valuable quantitative tradeoff information for competing treatment policies was able to be determined, and optimal sequences were projected.

\subsection{Discussion and Future Work}

The D-MODT methodology provides a patient-specific, multiobjective approach to modeling the efficacious impacts of HCC treatments. To further advance the state of the art, there are many future directions for this research:

Improvement of model structure. As was evidenced in section 5.1, every additional month considered in the D-MODT results in an exponentially increasing number of computations to be performed. This fact, while not important for small models such as the one presented in this paper's case study, becomes a major problem when attempting to chart out and solve a larger model, such as one for a priority MELD patient in Region 5 (CA, NV, UT, NM, AZ, HI), who has an expected wait time of 16 months until transplantation. The D-MODT's robustness, in this sense, is a weakness: because the methodology accounts for every possible combination of therapies and probabilistic states of health a patient may encounter during his treatment. Folding back at every nonterminal decision node, every permutation of Pareto-optimal treatment policies from the last period must be considered. In this paper, seven arcs were charted out from every chance node, which lead to a staggering 192 permutations of treatment policies at the first non-terminal decision node. 
What Markov models lack in robustness and specificity, they certainly make up for in convenience. Future attempts at refining this methodology should consider integrating the Markov state transition diagram within the D-MODT, while still maintaining the D-MODT's Bayesian mathematics. The D-MODT was developed as a complementary method to Markov models, and the strengths of the two approaches should be attempted to be combined into a single model.

Improved definition of the state variable. Haimes (2012) notes that the state variable characterizes the system as a whole and plays a major role in estimating its future behavior for any given inputs. In the context of HCC treatment, it is important to define an appropriate state variable so that a patient's progress may be tracked throughout the management of his disease. This approach uses the MELD score as that state variable. While the MELD score is a valuable measure with which physicians manage the allocation of donor organs, MELD is not in itself a true "state variable," as MELD is a calculated mathematical combination of three biochemical factors: bilirubin, Creatinine, and INR. A true state variable should be observable, which MELD is not; a physician should prescribe a therapy based off of specific observations. Thus, work must be done on determining an appropriate indicator of the severity of HCC within a patient. Alphafetoprotein has been discussed as a candidate for this indicator, and is conveniently accounted for in the STAR database (as a field in the HCC_TUMOR_DATA dataset). Other candidates include the magnitude of tumor volume between scans, and tumor staging levels. Studies should be conducted using these various factors as state variables rather than MELD. 
Development of complementary software. The D-MODT's ability to project efficacious treatment sequences at any checkup should be incorporated into an application (i.e., iPhone application) that physicians could use to extract tradeoff information on-the-go. This future aim is currently part of a pending patent by the author. This would allow for widespread use of the D-MODT methodology in clinical settings and promote further work between engineering and medicine.

Further data collection. For this model's case study, data from the STAR database was used wherever it was available. However, even in a one month example, there were many limitations presented by the database. The final vectors at the end of the decision tree were populated with data from the literature and assumptions made by the other rather than observed from patient records in the database, because these records were insufficient in number. Also, data regarding the effects of administering combinations of different therapies must be collected and studied, as the D-MODT allows for the possibility that a physician may prescribe a different therapy to his patient at any given month.

Extension to other areas. The success of this and future modeling efforts should promote the use of this approach in contexts other than HCC treatment. Other disease management situations, such as kidney disease, are characterized by similar organ allocation dilemmas. Physicians involved in these decision-making processes certainly also face treatment schedules where they observe a patient on a regular basis, take a measurement of his state variable, and must decide among competing options for his next 
therapy. It is beyond human capability to accurately decide among many alternatives, which necessitates the development and use of auxiliary decision-making technology, such as the D-MODT.

\subsection{Conclusion}

Physicians managing hepatocellular carcinoma (HCC) patients awaiting orthotopic liver transplantation (OLT) are faced with a myriad of treatment options. As transplantation requires lengthy waiting times in some regions of the United States, it is important that the physician best sequence his patient's bridging therapies to maintain his state of health until a donor liver becomes available. The heterogeneity of the HCC patient cohort and their rapidly changing states of health create a decision-making problem where, given the knowledge of a patient's current and past states, the physician must make a decision among the many therapies available at each checkup. The socalled "seven plus-minus-two" rule prohibits a human from accurately judging between many such options.

This thesis builds upon prior work to develop a dynamic multiobjective decision tree (D-MODT) methodology capable of elucidating quantitative tradeoff information for therapies available to the patient at each state throughout his overall treatment. The Bayesian mathematics and patient specificity built into this approach seek to extend the current state of the art by complementing the widely-used Markov decision models.

The D-MODT methodology must continue to be refined and improved by future researchers. The ability to employ the robust mathematics of Bayesian analysis and still maintain a high level of patient specificity cause the current methodology to be 
computationally expensive. The simplicity and quick simulation ability associated with Markov models should be integrated into the D-MODT; namely, the transitions of a patient within the "state of health" space could be integrated into a state transition diagram that would eliminate the need for the cumbersome chance nodes that the DMODT currently contains. Furthermore, more appropriate (i.e., observable) state variables should be considered with which to indicate a patient's level of disease progression or regression during his treatment.

The current work should be regarded as the laying of a mathematical and theoretical foundation with which to promote future data collection and from which to develop more refined models. With such models, it is the ultimate goal of this research to improve the process of HCC treatment for all parties concerned. 


\section{REFERENCES}

Altekruse S, McGlynn K, Reichman M. Hepatocellular carcinoma incidence, mortality, and survival trends in the United States from 1975 to 2005. Journal of Clinical Oncology 2009;27:1485-1491.

Azoulay D, et al. Decision for retransplantation of the liver: an experience and costbased analysis. Annals of Surgery 2002;236:713-721.

Bleistein, J. Hepatocellular carcinoma treatment decision analysis: a multi-objective decision tree approach (Master's thesis). University of Virginia, Charlottesville, VA. 2011.

Bruix J, Sherman M. Management of hepatocellular carcinoma: an update. Hepatology 2005; 42(5): 1208-1236.

Chen C. Linear System Theory and Design. $3^{\text {rd }}$ Edition. 1999 Oxford University Press: New York.

Cheng S, et al. Living-donor versus cadaveric liver transplantation for non-resectable small hepatocellular carcinoma and compensated cirrhosis: a decision analysis.

Transplantation 2001;72:861-868.

Choi D, et al. Percutaneous radiofrequency ablation for early-stage hepatocellular carcinoma as a first-line treatment: long-term results and prognostic factors in a large single-institution series. European Radiology 2007; 17: 684-692.

Chok KS, et al. Comparable survival in patients with unresectable hepatocellular carcinoma treated by radiofrequency ablation or transarterial chemoembolization. Archives of Surgery 2006; 141: 1231-1236.

Davis, G, et al. Aging of hepatitis C virus (HCV)-infected persons in the United States: a multiple cohort model of $\mathrm{HCV}$ prevalence and disease progression. Gastroenterology 2010; 138(2): 513-521. 
El-Serag H. Hepatocellular carcinoma: recent trends in the United States.

Gastroenterology 2004;127:S27-S34.

Graziadei IW, et al. Chemoembolization followed by liver transplantation for hepatocellular carcinoma impedes tumor progression while on the waiting list and leads to excellent outcome. Liver Transplantation 2003; 9(6): 557-563.

Haimes YY. Risk Modeling, Assessment, and Management. $3^{\text {rd }}$ Edition. 2009 John Wiley \& Sons, Inc.: Hoboken, NJ.

Haimes YY. Modeling complex systems of systems with phantom systems models. Systems Engineering 2012; 15(3): 333-346.

Haimes YY. Systems-based guiding principles for risk modeling, planning, assessment, management, and communication. Risk Analysis 2012; 32(9): 1451-1467.

Haimes YY, Li D, and Tulsiani V. Multiobjective decision-tree analysis. Risk Analysis 1990; 10:111-127.

Hashikura Y, et al. Long-term results of living-related donor liver graft transplantation: A single-center analysis of 110 transplants. Transplantation 2001;72:95-99.

Kamath P, Kim W. The model for end-stage liver disease (MELD). Hepatology 2007; 45(3): 797-805.

Kulik, et al. Outcomes of living and deceased donor liver transplant recipients with hepatocellular carcinoma: results of the A2ALL cohort. American Journal of Transplantation 2012; 12(11): 2997-3007.

Lau WY and Lai ECH. The current role of radiofrequency ablation in the management of hepatocellular carcinoma. Annals of Surgery 2009;249:20-25. 
Livraghi T, et al. No treatment, resection and ethanol injection in hepatocellular carcinoma: a retrospective analysis of survival in 391 patients with cirrhosis. Journal of Hepatology 1995;22:522-526

Llovet JM, Burroughs A, Bruix J. Hepatocellular carcinoma. Lancet 2003;362:19071917.

Llovet JM, Fuster J, Bruix J. The Barcelona approach: diagnosis, staging, and treatment of hepatocellular carcinoma. Liver Transplantation 2004;10:S115-S120.

Llovet JM, et al. Cost effectiveness of adjuvant therapy for hepatocellular carcinoma during the waiting list for liver transplantation. Gut 2002;50:123-128.

Llovet JM, et al. Sorafenib in advanced hepatocellular carcinoma. New England Journal of Medicine 2008;359:378-390.

Lu DS, et al. Percutaneous radiofrequency ablation of hepatocellular carcinoma as a bridge to liver transplantation. Hepatology 2005; 41(5): 1130-1137.

Maddala YK, et al. Drop-out rates of patients with hepatocellular cancer listed for liver transplantation: outcome with chemoembolization. Liver Transplantation 2004; 10(3): 449-455.

Markmann JF, et al. Long-term survival after retransplantation of the liver. Annals of Surgery 1997;226:408-420.

Mazzaferro V, et al. Liver transplantation for the treatment of small hepatocellular carcinomas in patients with cirrhosis. New England Journal of Medicine 1996;334:693699.

Merion RM, et al. The survival benefit of liver transplantation. American Journal of Transplantation 2005; 5(2): 307-313. 
Miller G. The magical number seven, plus or minus two: some limits on our capacity for processing information. The Psychological Review 1956; 63: 81-97.

Naugler WE, Sonnenberg A. Survival and cost-effectiveness analysis of competing strategies in the management of small hepatocellular carcinoma. Liver Transplantation 2010;16:1186-1194.

Nomura F, Ohnishi K, Tanabe Y. Clinical features and prognosis of hepatocellular carcinoma with reference to serum alpha-fetoprotein levels: analysis of 606 patients. Cancer 1989; 64(8): 1700-1707.

Northup PG, et al. Addition of adult-to-adult living donation to liver transplant programs improves survival but at an increased cost. Liver Transplantation 2009;15:148-162.

Oldhafer KJ, et al. Arterial chemoembolization before liver transplantation in patients with hepatocellular carcinoma: marked tumor necrosis, but no survival benefit? Journal of Hepatology 1998; 29: 953-959.

Organ Procurement and Transplantation Network. http://optn.transplant.hrsa.gov/latestData/rptStrat.asp. 17 January 2013.

Parkin DM, et al. Estimating the world cancer burden: Globocan 2000. International Journal of Cancer 2001; 94(2): 153-156.

Raiffa H. Decision Analysis: Introductory Lectures on Choice Under Uncertaity. 1968 Addision-Wesley: Reading, MA.

Raut CP, et al. Significant long-term survival after radiofrequency ablation of unresectable hepatocellular carcinoma in patients with cirrhosis. Annals of Surgical Oncology 2005; 12(8): 616-628.

Roberts JP, et al. Hepatocellular carcinoma: ablate and wait versus rapid transplantation. Liver Transplantation 2010;16:925-929. 
Rodriguez-Luna $\mathrm{H}$, et al. Hepatitis $\mathrm{C}$ virus recurrence in living donor liver transplant recipients. Digestive Diseases and Sciences 2004;49:38-41.

Salem R, et al. Radioembolization for hepatocellular carcinoma Using yttrium-90 microspheres: a comprehensive report of long-term outcomes. Gastroenterology 2010;138:52-64.

Sangiovanni A, et al. The natural history of compensated cirrhosis due to hepatitis C virus: a 17-year cohort study of 214 patients. Hepatology 2006;43:1303-1310.

Sarasin F, et al. Living donor liver transplantation for early hepatocellular carcinoma: a life-expectancy and cost-effectiveness perspective. Hepatology 2001;33:1073-1079.

Takayasu K, et al. Prospective cohort study of transarterial chemoembolization for unresectable hepatocellular carcinoma in 8510 patients. Gastroenterology 2006; 131: 461-469.

Vitale A, et al. Use of sorafenib in patients with hepatocellular carcinoma before liver transplantation: a cost-benefit analysis while awaiting data on sorafenib safety. Hepatology 2010;51:165-173.

Volk ML. Unfair priority for HCC: a problem whose ideal solution remains unsolved. American Journal of Transplantation 2010;10:1507-1508.

Volk ML, Vijan S, and Marrero JA. A novel model measuring the harm of transplanting hepatocellular carcinoma exceeding Milan criteria. American Journal of Transplantation 2008; 8: 839-846.

Wiesner R, et al. Model for end-stage liver disease (MELD) and allocation of donor livers. Gastroenterology 2003;124:91-96. 


\section{ACKNOWLEDGMENT}

This work was supported in part by Health Resources and Services Administration contract 234-2005-370011C, the Dean's office of the School of Engineering and Applied Science, and the Center for Risk Management of Engineering Systems, University of Virginia. The content is the responsibility of the authors alone and does not necessarily reflect the views or policies of the Department of Health and Human Services, nor does mention of trade names, commercial products, or organizations imply endorsement by the U.S. Government. 


\section{APPENDIX A: MEDIAN WAIT TIMES BY MELD AND REGION}

The following table lists the median wait times for OPTN regions 1-11 based off of a patient's MELD score at listing. This data was harvested last on January 17, 2013, but can be updated by visiting: http://optn.transplant.hrsa.gov/latestData/rptStrat.asp. A “_“ indicates that an insufficient sample was available for inclusion in the table.

\begin{tabular}{|c|c|c|c|c|}
\hline REGION & INIT MELD & $\begin{array}{l}\text { MEDIAN } \\
\text { WAIT (D) }\end{array}$ & $\begin{array}{l}\text { NEAREST } \\
\text { MONTHS }\end{array}$ & 95\% CONF \\
\hline \multirow[t]{4}{*}{1} & $(, 10)$ & - & - & - \\
\hline & {$[11,18]$} & 1971 & 66 & - \\
\hline & {$[19,24]$} & 213 & 7 & $(157,371)$ \\
\hline & {$[25)$,} & 38 & 1 & $(33,59)$ \\
\hline \multirow[t]{4}{*}{2} & $(, 10)$ & 2777 & 93 & $(1628,-)$ \\
\hline & {$[11,18]$} & 631 & 21 & $(500,812)$ \\
\hline & {$[19,24]$} & 111 & 4 & $(91,133)$ \\
\hline & {$[25)$,} & 29 & 1 & $(19,36)$ \\
\hline \multirow[t]{4}{*}{3} & $(, 10)$ & 177 & 6 & $(126,284)$ \\
\hline & {$[11,18]$} & 157 & 5 & $(122,184)$ \\
\hline & {$[19,24]$} & 49 & 2 & $(38,55)$ \\
\hline & {$[25)$,} & 9 & 1 & $(8,12)$ \\
\hline \multirow[t]{4}{*}{4} & $(, 10)$ & 2433 & 81 & $(1462,)$. \\
\hline & {$[11,18]$} & 826 & 28 & $(561,982)$ \\
\hline & {$[19,24]$} & 75 & 3 & $(44,105)$ \\
\hline & {$[25)$,} & 14 & 1 & $(10,22)$ \\
\hline \multirow[t]{4}{*}{5} & $(, 10)$ & 3564 & 119 & $(2885,)$. \\
\hline & {$[11,18]$} & 2277 & 76 & $(1923,2852)$ \\
\hline & {$[19,24]$} & 490 & 16 & $(334,735)$ \\
\hline & {$[25)$,} & 35 & 1 & $(24,45)$ \\
\hline \multirow[t]{4}{*}{6} & $(, 10)$ & 441 & 15 & $(229,1250)$ \\
\hline & {$[11,18]$} & 296 & 10 & $(198,450)$ \\
\hline & {$[19,24]$} & 40 & 1 & $(28,88)$ \\
\hline & {$[25)$,} & 10 & 1 & $(5,20)$ \\
\hline \multirow[t]{4}{*}{7} & $(, 10)$ & 1120 & 37 & $(598,2044)$ \\
\hline & {$[11,18]$} & 513 & 17 & $(426,620)$ \\
\hline & {$[19,24]$} & 126 & 4 & $(89,149)$ \\
\hline & {$[25)$,} & 14 & 1 & $(11,18)$ \\
\hline \multirow[t]{3}{*}{8} & $(, 10)$ & 1091 & 36 & $(583,2017)$ \\
\hline & {$[11,18]$} & 530 & 18 & $(411,815)$ \\
\hline & {$[19,24]$} & 127 & 4 & $(77,165)$ \\
\hline
\end{tabular}




\begin{tabular}{|l|l|l|l|l|}
\hline & {$[25)$,} & 25 & 1 & $(20,45)$ \\
\hline 9 & $(, 10)$ & 3245 & 108 & $(2042,)$. \\
\hline & {$[11,18]$} & 947 & 32 & $(663,1298)$ \\
\hline & {$[19,24]$} & 211 & 7 & $(153,309)$ \\
\hline 10 & {$[25)$,} & 26 & 1 & $(19,35)$ \\
\hline & $(, 10)$ & 181 & 6 & $(119,291)$ \\
\hline & {$[11,18]$} & 140 & 5 & $(114,179)$ \\
\hline & {$[19,24]$} & 27 & 1 & $(21,36)$ \\
\hline 11 & {$[25)$,} & 15 & 1 & $(9,21)$ \\
\hline & $(, 10)$ & 2911 & 97 & $(2328,)$. \\
\hline & {$[11,18]$} & 805 & 27 & $(655,1051)$ \\
\hline & {$[19,24]$} & 98 & 3 & $(80,137)$ \\
\hline & {$[25)$,} & 22 & 1 & $(18,31)$ \\
\hline
\end{tabular}




\section{APPENDIX B: INDICES OF DATASET VARIABLES}

\section{LIVER_PUBLIC_USE}

\begin{tabular}{|l|l|}
\hline Name & Description \\
\hline ADMISSION_DATE & Date of Admission to TX Center \\
\hline CREAT_DIS & Serum Creatinine at Time of Discharge \\
\hline CREAT_TX & Serum Creatinine at Time of TX \\
\hline DAYSWAIT_CHRON & Days on Liver Waiting List \\
\hline DEATH_DATE & $\begin{array}{l}\text { Date of Death for Patient that Died on Waiting } \\
\text { List }\end{array}$ \\
\hline DIS_INR & INR at Discharge \\
\hline DON_TY & Donor Type-Deceased, Living \\
\hline END_DATE & $\begin{array}{l}\text { Earliest Dates of Removal From waiting List, } \\
\text { Transplant, Death }\end{array}$ \\
\hline END_STAT & Candidate Status at Transplant Offer \\
\hline EXC_HCC & Type of Exception Relative to HCC \\
\hline FINAL_BILIRUBIN & Most Recent Waiting List Bilirubin \\
\hline FINAL_INR & Most Recent Waiting List INR \\
\hline FINAL_MELD_OR_PELD & Most Recent Waiting List MELD or PELD \\
\hline FINAL_MELD_PELD_LAB_SCOREE & $\begin{array}{l}\text { Most Recent Waiting List MELD/PELD Lab } \\
\text { Score }\end{array}$ \\
\hline FINAL_SERUM_CREAT & Most Recent Waiting List Serum Creatinine \\
\hline INIT_BILIRUBIN & Initial Waiting List Bilirubin \\
\hline INIT_DATE & Beginning Date for Registration \\
\hline INIT_INR & Initial Waiting List INR \\
\hline INIT_MELD_OR_PELD & Initial Waiting List MELD or PELD \\
\hline INIT_MELD_PELD_LAB_SCORE & Initial Waiting List MELD/PELD Lab Score \\
\hline INIT_SERUM_CREAT & Initial Waiting List Serum Creatinine \\
\hline INIT_STAT & Initial Waiting List Status Code \\
\hline INR_TX & Recipient INR at Transplant \\
\hline LIST_MELD & Patient Listed Prior to MELD/PELD? \\
\hline LISTRY & Actual Year Registrant Listed \\
\hline MELD_PELD_LAB_SCORE & MELD/PELD Lab Score at Time of Transplant \\
\hline MOST_RCNT_CREAT & Recipient Most Recent Creatinine \\
\hline NUM_PREV_TX & The Number of Previous Transplants \\
\hline PSTATUS & Recipient Died (1=Dead, 0=Alive) \\
\hline PT_CODE & Encrypted Recipient Identifier \\
\hline PX_STAT & Recipient Status (Died, ReTX, Lost, Alive) \\
\hline PX_STAT_DATE & Recipient Status Date \\
\hline REGION & $\begin{array}{l}\text { WL UNOS/OPTN Region Where } \\
\text { Listed/Transplanted }\end{array}$ \\
\hline REM_CD & Reason for Removal from the Waiting List \\
\hline SSDMF_DEATH_DATE & Social Security Death Master File Death Date \\
\hline & \\
\hline
\end{tabular}




\begin{tabular}{|l|l|}
\hline TBILI_TX & Recipient Total Bilirubin @ Transplant \\
\hline TRR_ID_CODE & Encrypted Transplant Identifier \\
\hline TX_DATE & Transplant Date \\
\hline TX_MELD & Transplant Occurred Prior to MELD/PELD? \\
\hline TX_YEAR & Transplant Year \\
\hline WL_ID_CODE & Encrypted Registration Identifier \\
\hline
\end{tabular}

\section{HCC_TUMOR DATA}

\begin{tabular}{|l|l|}
\hline Name & Description \\
\hline ABLATE & Any Ablative Therapy (Y/N) \\
\hline CASE_ID & Case Number \\
\hline CHEM_ABLAT & Chemical Ablation of Lesion \\
\hline CHEMOEMBO & Chemoembolization of Lesion \\
\hline CRYO & Cryo Ablation of Lesion \\
\hline FORM_ID & Form ID \\
\hline HCC_DIAG & HCC Diagnosis \\
\hline INIT_EXT & Initial/Extension/Appeal \\
\hline ORIG_APPL_DATE & Application Date \\
\hline RESECT & Surgical Resection \\
\hline RFA & Radio Frequency Ablation of Lesion \\
\hline WL_ID_CODE & Encrypted Registration Identifier \\
\hline
\end{tabular}


APPENDIX C: PROBABILITY TABLES

\begin{tabular}{|l|l|l|l|l|l|l|l|}
\hline Therapy & P(De|M1) & P(M1|M1) & P(M2|M1) & P(M3|M1) & P(M4|M1) & P(M5|M1) & P(Dr|M1) \\
\hline TACE & 3.37 & 65.11 & 22.73 & 5.68 & 1.65 & 0.22 & 1.24 \\
\hline RFA & 4.14 & 67.64 & 21.33 & 4.31 & 1.29 & 0.43 & 0.86 \\
\hline DN & 5.09 & 61.87 & 25.33 & 4.93 & 1.60 & 0.37 & 0.81 \\
\hline
\end{tabular}

Chance Node Probabilities For Initial Meld [0,10)

\begin{tabular}{|l|l|l|l|l|l|l|l|}
\hline Therapy & P(De|M2) & P(M1 $\mid$ M2 $)$ & P(M2|M2) & P(M3|M2) & P(M4|M2) & P(M5|M2) & P(Dr|M2) \\
\hline TACE & 3.37 & 12.57 & 56.91 & 19.06 & 4.96 & 1.89 & 1.24 \\
\hline RFA & 4.14 & 10.95 & 60.67 & 18.54 & 4.00 & 0.84 & 0.86 \\
\hline DN & 5.09 & 10.11 & 58.38 & 20.08 & 4.01 & 1.52 & 0.81 \\
\hline
\end{tabular}

Chance Node Probabilities For Initial Meld [10,15)

\begin{tabular}{|l|l|l|l|l|l|l|l|}
\hline Therapy & P(De|M3) & P(M1|M3) & P(M2|M3) & P(M3|M3) & P(M4|M3) & P(M5|M3) & P(Dr|M3) \\
\hline TACE & 3.37 & 1.42 & 21.54 & 53.97 & 15.62 & 2.84 & 1.24 \\
\hline RFA & 4.14 & 2.59 & 21.97 & 54.93 & 11.63 & 3.88 & 0.86 \\
\hline DN & 5.09 & 0.97 & 16.68 & 59.34 & 14.62 & 2.49 & 0.81 \\
\hline
\end{tabular}

Chance Node Probabilities For Initial Meld $[15,20)$

\begin{tabular}{|l|l|l|l|l|l|l|l|}
\hline Therapy & P(De|M4) & P(M1|M4) & P(M2|M4) & P(M3|M4) & P(M4|M4) & P(M5|M4) & P(Dr|M4) \\
\hline TACE & 3.37 & $<0.00001$ & 9.54 & 23.17 & 55.87 & 6.81 & 1.24 \\
\hline RFA & 4.14 & $<0.00001$ & 7.31 & 47.50 & 40.19 & $<0.00001$ & 0.86 \\
\hline DN & 5.09 & 0.60 & 6.59 & 26.97 & 53.34 & 6.59 & 0.81 \\
\hline
\end{tabular}

Chance Node Probabilities For Initial Meld [20,25)

\begin{tabular}{|l|l|l|l|l|l|l|l|}
\hline Therapy & P(De|M5) & P(M1 $\mid$ M5) & P(M2|M5) & P(M3|M5) & P(M4|M5) & P(M5|M5) & P(Dr|M5) \\
\hline TACE & 3.37 & $<0.00001$ & 27.25 & 40.88 & 27.25 & $<0.00001$ & 1.24 \\
\hline RFA & 4.14 & $<0.00001$ & $<0.00001$ & 95.00 & $<0.00001$ & $<0.00001$ & 0.86 \\
\hline DN & 5.09 & $<0.00001$ & 16.61 & 22.14 & 27.67 & 27.67 & 0.81 \\
\hline
\end{tabular}

Chance Node Probabilities For Initial Meld 25+ 


\section{APPENDIX D: DECISIONS FOR MONTH 0 NODES}

\section{Initial Calculated Meld $<10$}

\begin{tabular}{|c|c|c|c|c|c|c|c|c|c|c|}
\hline \multirow{3}{*}{$\begin{array}{c}\text { Policy } \\
\# \\
1\end{array}$} & \multicolumn{4}{|c|}{$\begin{array}{l}\text { Month } 1 \\
\text { Decision }\end{array}$} & \multirow[b]{2}{*}{ M4 } & \multirow[b]{2}{*}{ M5 } & \multirow[b]{2}{*}{ Drop } & \multicolumn{2}{|c|}{ Damage Vector } & \multirow[b]{2}{*}{$\mathrm{P}-\mathrm{O}$} \\
\hline & $\begin{array}{c}\text { Initial } \\
\text { Decision }\end{array}$ & M1 & M2 & M3 & & & & Lives & Cost & \\
\hline & TACE & $\mathrm{TX}$ & $\mathrm{TX}$ & $\mathrm{TX}$ & $\mathrm{TX}$ & $\mathrm{TX}$ & Sorafenib & 77.72 & 271121.30 & \\
\hline 2 & TACE & TX & TX & TX & $\mathrm{TX}$ & TX & $\mathrm{DN}$ & 77.68 & 271062.86 & \\
\hline 3 & TACE & $\mathrm{TX}$ & $\mathrm{TX}$ & $\mathrm{TX}$ & $\mathrm{TX}$ & TACE & Sorafenib & 77.65 & 270563.17 & \\
\hline 4 & TACE & $\mathrm{TX}$ & $\mathrm{TX}$ & $\mathrm{TX}$ & $\mathrm{TX}$ & TACE & $\mathrm{DN}$ & 77.61 & 270504.73 & \\
\hline 5 & TACE & $\mathrm{TX}$ & $\mathrm{TX}$ & $\mathrm{TX}$ & TACE & $\mathrm{TX}$ & Sorafenib & 77.22 & 266935.32 & $\mathrm{X}$ \\
\hline 6 & TACE & $\mathrm{TX}$ & $\mathrm{TX}$ & $\mathrm{TX}$ & TACE & $\mathrm{TX}$ & $\mathrm{DN}$ & 77.19 & 266876.87 & $\mathrm{X}$ \\
\hline 7 & TACE & $\mathrm{TX}$ & $\mathrm{TX}$ & $\mathrm{TX}$ & TACE & TACE & Sorafenib & 77.16 & 266377.19 & $\mathrm{X}$ \\
\hline 8 & TACE & $\mathrm{TX}$ & $\mathrm{TX}$ & $\mathrm{TX}$ & TACE & TACE & $\mathrm{DN}$ & 77.12 & 266318.74 & $\mathrm{X}$ \\
\hline 9 & TACE & $\mathrm{TX}$ & $\mathrm{TX}$ & TACE & $\mathrm{TX}$ & $\mathrm{TX}$ & Sorafenib & 76.02 & 256711.37 & $\mathrm{X}$ \\
\hline 10 & TACE & $\mathrm{TX}$ & $\mathrm{TX}$ & TACE & $\mathrm{TX}$ & $\mathrm{TX}$ & DN & 75.99 & 256652.93 & $\mathrm{X}$ \\
\hline 11 & TACE & $\mathrm{TX}$ & $\mathrm{TX}$ & TACE & $\mathrm{TX}$ & TACE & Sorafenib & 75.96 & 256153.24 & $\mathrm{X}$ \\
\hline 12 & TACE & $\mathrm{TX}$ & $\mathrm{TX}$ & TACE & $\mathrm{TX}$ & TACE & DN & 75.92 & 256094.79 & $\mathrm{X}$ \\
\hline 13 & TACE & $\mathrm{TX}$ & TX & TACE & TACE & $\mathrm{TX}$ & Sorafenib & 75.53 & 252525.39 & $\mathrm{X}$ \\
\hline 14 & TACE & $\mathrm{TX}$ & TX & TACE & TACE & TX & $\mathrm{DN}$ & 75.50 & 252466.94 & $\mathrm{X}$ \\
\hline 15 & TACE & $\mathrm{TX}$ & TX & TACE & TACE & TACE & Sorafenib & 75.47 & 251967.26 & $\mathrm{X}$ \\
\hline 16 & TACE & $\mathrm{TX}$ & TX & TACE & TACE & TACE & $\mathrm{DN}$ & 75.43 & 251908.81 & $\mathrm{X}$ \\
\hline 17 & TACE & $\mathrm{TX}$ & TACE & $\mathrm{TX}$ & $\mathrm{TX}$ & $\mathrm{TX}$ & Sorafenib & 70.95 & 213456.20 & $\mathrm{X}$ \\
\hline 18 & TACE & $\mathrm{TX}$ & TACE & TX & $\mathrm{TX}$ & $\mathrm{TX}$ & $\mathrm{DN}$ & 70.91 & 213397.76 & $\mathrm{X}$ \\
\hline 19 & TACE & $\mathrm{TX}$ & TACE & $\mathrm{TX}$ & $\mathrm{TX}$ & TACE & Sorafenib & 70.88 & 212898.07 & $\mathrm{X}$ \\
\hline 20 & TACE & $\mathrm{TX}$ & TACE & $\mathrm{TX}$ & $\mathrm{TX}$ & TACE & $\mathrm{DN}$ & 70.85 & 212839.63 & $\mathrm{X}$ \\
\hline 21 & TACE & $\mathrm{TX}$ & TACE & $\mathrm{TX}$ & TACE & $\mathrm{TX}$ & Sorafenib & 70.46 & 209270.22 & $\mathrm{X}$ \\
\hline 22 & TACE & $\mathrm{TX}$ & TACE & $\mathrm{TX}$ & TACE & $\mathrm{TX}$ & DN & 70.42 & 209211.77 & $\mathrm{X}$ \\
\hline 23 & TACE & $\mathrm{TX}$ & TACE & $\mathrm{TX}$ & TACE & TACE & Sorafenib & 70.39 & 208712.09 & $\mathrm{X}$ \\
\hline 24 & TACE & $\mathrm{TX}$ & TACE & $\mathrm{TX}$ & TACE & TACE & $\mathrm{DN}$ & 70.36 & 208653.64 & $\mathrm{X}$ \\
\hline 25 & TACE & $\mathrm{TX}$ & TACE & TACE & $\mathrm{TX}$ & $\mathrm{TX}$ & Sorafenib & 69.26 & 199046.27 & $\mathrm{X}$ \\
\hline 26 & TACE & $\mathrm{TX}$ & TACE & TACE & $\mathrm{TX}$ & TX & $\mathrm{DN}$ & 69.22 & 198987.83 & $\mathrm{X}$ \\
\hline 27 & TACE & $\mathrm{TX}$ & TACE & TACE & $\mathrm{TX}$ & TACE & Sorafenib & 69.19 & 198488.14 & $\mathrm{X}$ \\
\hline 28 & TACE & $\mathrm{TX}$ & TACE & TACE & $\mathrm{TX}$ & TACE & DN & 69.16 & 198429.69 & $\mathrm{X}$ \\
\hline 29 & TACE & $\mathrm{TX}$ & TACE & TACE & TACE & $\mathrm{TX}$ & Sorafenib & 68.77 & 194860.29 & \\
\hline 30 & TACE & $\mathrm{TX}$ & TACE & TACE & TACE & $\mathrm{TX}$ & DN & 68.73 & 194801.84 & \\
\hline 31 & TACE & $\mathrm{TX}$ & TACE & TACE & TACE & TACE & Sorafenib & 68.70 & 194302.16 & \\
\hline 32 & TACE & $\mathrm{TX}$ & TACE & TACE & TACE & TACE & DN & 68.66 & 194243.71 & \\
\hline 33 & TACE & TACE & $\mathrm{TX}$ & $\mathrm{TX}$ & $\mathrm{TX}$ & $\mathrm{TX}$ & Sorafenib & 58.33 & 105939.84 & $\mathrm{X}$ \\
\hline 34 & TACE & TACE & $\mathrm{TX}$ & $\mathrm{TX}$ & $\mathrm{TX}$ & $\mathrm{TX}$ & $\mathrm{DN}$ & 58.30 & 105881.39 & $\mathrm{X}$ \\
\hline 35 & TACE & TACE & $\mathrm{TX}$ & $\mathrm{TX}$ & $\mathrm{TX}$ & TACE & Sorafenib & 58.27 & 105381.71 & $\mathrm{X}$ \\
\hline 36 & TACE & TACE & $\mathrm{TX}$ & $\mathrm{TX}$ & $\mathrm{TX}$ & TACE & $\mathrm{DN}$ & 58.23 & 105323.26 & $\mathrm{X}$ \\
\hline 37 & TACE & TACE & TX & $\mathrm{TX}$ & TACE & $\mathrm{TX}$ & Sorafenib & 57.84 & 101753.85 & $\mathrm{X}$ \\
\hline
\end{tabular}




\begin{tabular}{|c|c|c|c|c|c|c|c|c|c|}
\hline 38 & TACE & TACE & $\mathrm{TX}$ & $\mathrm{TX}$ & TACE & $\mathrm{TX}$ & $\mathrm{DN}$ & 57.80 & 101695.41 \\
\hline 39 & TACE & TACE & $\mathrm{TX}$ & $\mathrm{TX}$ & TACE & TACE & Sorafenib & 57.78 & 101195.72 \\
\hline 40 & TACE & TACE & $\mathrm{TX}$ & $\mathrm{TX}$ & TACE & TACE & DN & 57.74 & 101137.28 \\
\hline 41 & TACE & TACE & $\mathrm{TX}$ & TACE & $\mathrm{TX}$ & $\mathrm{TX}$ & Sorafenib & 56.64 & 91529.91 \\
\hline 42 & TACE & TACE & $\mathrm{TX}$ & TACE & $\mathrm{TX}$ & $\mathrm{TX}$ & DN & 56.60 & 91471.46 \\
\hline 43 & TACE & TACE & $\mathrm{TX}$ & TACE & $\mathrm{TX}$ & TACE & Sorafenib & 56.58 & 90971.77 \\
\hline 44 & TACE & TACE & $\mathrm{TX}$ & TACE & $\mathrm{TX}$ & TACE & DN & 56.54 & 90913.33 \\
\hline 45 & TACE & TACE & $\mathrm{TX}$ & TACE & TACE & $\mathrm{TX}$ & Sorafenib & 56.15 & 87343.92 \\
\hline 46 & TACE & TACE & $\mathrm{TX}$ & TACE & TACE & $\mathrm{TX}$ & DN & 56.11 & 87285.48 \\
\hline 47 & TACE & TACE & $\mathrm{TX}$ & TACE & TACE & TACE & Sorafenib & 56.08 & 86785.79 \\
\hline 48 & TACE & TACE & $\mathrm{TX}$ & TACE & TACE & TACE & DN & 56.05 & 86727.35 \\
\hline 49 & TACE & TACE & TACE & $\mathrm{TX}$ & $\mathrm{TX}$ & $\mathrm{TX}$ & Sorafenib & 51.57 & 48274.74 \\
\hline 50 & TACE & TACE & TACE & $\mathrm{TX}$ & $\mathrm{TX}$ & $\mathrm{TX}$ & DN & 51.53 & 48216.29 \\
\hline 51 & TACE & TACE & TACE & $\mathrm{TX}$ & $\mathrm{TX}$ & TACE & Sorafenib & 51.50 & 47716.61 \\
\hline 52 & TACE & TACE & TACE & $\mathrm{TX}$ & $\mathrm{TX}$ & TACE & DN & 51.46 & 47658.16 \\
\hline 53 & TACE & TACE & TACE & $\mathrm{TX}$ & TACE & $\mathrm{TX}$ & Sorafenib & 51.07 & 44088.75 \\
\hline 54 & TACE & TACE & TACE & $\mathrm{TX}$ & TACE & $\mathrm{TX}$ & DN & 51.04 & 44030.31 \\
\hline 55 & TACE & TACE & TACE & $\mathrm{TX}$ & TACE & TACE & Sorafenib & 51.01 & 43530.62 \\
\hline 56 & TACE & TACE & TACE & $\mathrm{TX}$ & TACE & TACE & DN & 50.97 & 43472.18 \\
\hline 57 & TACE & TACE & TACE & TACE & $\mathrm{TX}$ & $\mathrm{TX}$ & Sorafenib & 49.87 & 33864.80 \\
\hline 58 & TACE & TACE & TACE & TACE & $\mathrm{TX}$ & $\mathrm{TX}$ & DN & 49.84 & 33806.36 \\
\hline 59 & TACE & TACE & TACE & TACE & $\mathrm{TX}$ & TACE & Sorafenib & 49.81 & 33306.67 \\
\hline 60 & TACE & TACE & TACE & TACE & $\mathrm{TX}$ & TACE & DN & 49.77 & 33248.23 \\
\hline 61 & TACE & TACE & TACE & TACE & TACE & $\mathrm{TX}$ & Sorafenib & 49.38 & 29678.82 \\
\hline 62 & TACE & TACE & TACE & TACE & TACE & $\mathrm{TX}$ & DN & 49.35 & 29620.38 \\
\hline 63 & TACE & TACE & TACE & TACE & TACE & TACE & Sorafenib & 49.32 & 29120.69 \\
\hline 64 & TACE & TACE & TACE & TACE & TACE & TACE & $\mathrm{DN}$ & 49.28 & 29062.24 \\
\hline 65 & RFA & $\mathrm{TX}$ & $\mathrm{TX}$ & $\mathrm{TX}$ & $\mathrm{TX}$ & $\mathrm{TX}$ & Sorafenib & 77.48 & 266347.78 \\
\hline 66 & RFA & $\mathrm{TX}$ & $\mathrm{TX}$ & $\mathrm{TX}$ & $\mathrm{TX}$ & $\mathrm{TX}$ & $\mathrm{DN}$ & 77.46 & 266307.25 \\
\hline 67 & RFA & $\mathrm{TX}$ & $\mathrm{TX}$ & $\mathrm{TX}$ & $\mathrm{TX}$ & RFA & Sorafenib & 77.41 & 265231.44 \\
\hline 68 & RFA & $\mathrm{TX}$ & $\mathrm{TX}$ & $\mathrm{TX}$ & $\mathrm{TX}$ & RFA & DN & 77.38 & 265190.91 \\
\hline 69 & RFA & $\mathrm{TX}$ & $\mathrm{TX}$ & $\mathrm{TX}$ & RFA & $\mathrm{TX}$ & Sorafenib & 77.25 & 262998.76 \\
\hline 70 & RFA & $\mathrm{TX}$ & $\mathrm{TX}$ & $\mathrm{TX}$ & RFA & $\mathrm{TX}$ & $\mathrm{DN}$ & 77.23 & 262958.22 \\
\hline 71 & RFA & $\mathrm{TX}$ & $\mathrm{TX}$ & $\mathrm{TX}$ & RFA & RFA & Sorafenib & 77.18 & 261882.42 \\
\hline 72 & RFA & $\mathrm{TX}$ & $\mathrm{TX}$ & $\mathrm{TX}$ & RFA & RFA & $\mathrm{DN}$ & 77.15 & 261841.88 \\
\hline 73 & RFA & $\mathrm{TX}$ & $\mathrm{TX}$ & RFA & $\mathrm{TX}$ & $\mathrm{TX}$ & Sorafenib & 76.72 & 255158.42 \\
\hline 74 & RFA & $\mathrm{TX}$ & $\mathrm{TX}$ & RFA & $\mathrm{TX}$ & $\mathrm{TX}$ & DN & 76.69 & 255117.88 \\
\hline 75 & RFA & $\mathrm{TX}$ & $\mathrm{TX}$ & RFA & $\mathrm{TX}$ & RFA & Sorafenib & 76.64 & 254042.08 \\
\hline 76 & RFA & $\mathrm{TX}$ & $\mathrm{TX}$ & RFA & $\mathrm{TX}$ & RFA & DN & 76.62 & 254001.54 \\
\hline 77 & RFA & $\mathrm{TX}$ & $\mathrm{TX}$ & RFA & RFA & $\mathrm{TX}$ & Sorafenib & 76.49 & 251809.40 \\
\hline 78 & RFA & $\mathrm{TX}$ & $\mathrm{TX}$ & RFA & RFA & $\mathrm{TX}$ & $\mathrm{DN}$ & 76.46 & 251768.86 \\
\hline 79 & RFA & $\mathrm{TX}$ & $\mathrm{TX}$ & RFA & RFA & RFA & Sorafenib & 76.41 & 250693.06 \\
\hline 80 & RFA & $\mathrm{TX}$ & $\mathrm{TX}$ & RFA & RFA & RFA & $\mathrm{DN}$ & 76.39 & 250652.52 \\
\hline 81 & RFA & $\mathrm{TX}$ & RFA & $\mathrm{TX}$ & $\mathrm{TX}$ & $\mathrm{TX}$ & Sorafenib & 73.70 & 210972.11 \\
\hline 82 & RFA & $\mathrm{TX}$ & RFA & $\mathrm{TX}$ & $\mathrm{TX}$ & $\mathrm{TX}$ & DN & 73.68 & 210931.58 \\
\hline 83 & RFA & $\mathrm{TX}$ & RFA & $\mathrm{TX}$ & $\mathrm{TX}$ & RFA & Sorafenib & 73.63 & 209855.77 \\
\hline
\end{tabular}




\begin{tabular}{|c|c|c|c|c|c|c|c|c|c|}
\hline 84 & RFA & $\mathrm{TX}$ & RFA & $\mathrm{TX}$ & $\mathrm{TX}$ & RFA & DN & 73.60 & 209815.24 \\
\hline 85 & RFA & $\mathrm{TX}$ & RFA & $\mathrm{TX}$ & RFA & $\mathrm{TX}$ & Sorafenib & 73.47 & 207623.09 \\
\hline 86 & RFA & $\mathrm{TX}$ & RFA & $\mathrm{TX}$ & RFA & $\mathrm{TX}$ & DN & 73.45 & 207582.56 \\
\hline 87 & RFA & $\mathrm{TX}$ & RFA & TX & RFA & RFA & Sorafenib & 73.40 & 206506.75 \\
\hline 88 & RFA & $\mathrm{TX}$ & RFA & TX & RFA & RFA & DN & 73.37 & 206466.22 \\
\hline 89 & RFA & $\mathrm{TX}$ & RFA & RFA & $\mathrm{TX}$ & $\mathrm{TX}$ & Sorafenib & 72.94 & 199782.75 \\
\hline 90 & RFA & $\mathrm{TX}$ & RFA & RFA & $\mathrm{TX}$ & $\mathrm{TX}$ & DN & 72.91 & 199742.22 \\
\hline 91 & RFA & $\mathrm{TX}$ & RFA & RFA & $\mathrm{TX}$ & RFA & Sorafenib & 72.86 & 198666.41 \\
\hline 92 & RFA & $\mathrm{TX}$ & RFA & RFA & $\mathrm{TX}$ & RFA & DN & 72.84 & 198625.88 \\
\hline 93 & RFA & $\mathrm{TX}$ & RFA & RFA & RFA & TX & Sorafenib & 72.71 & 196433.73 \\
\hline 94 & RFA & $\mathrm{TX}$ & RFA & RFA & RFA & $\mathrm{TX}$ & DN & 72.68 & 196393.20 \\
\hline 95 & RFA & $\mathrm{TX}$ & RFA & RFA & RFA & RFA & Sorafenib & 72.63 & 195317.39 \\
\hline 96 & RFA & $\mathrm{TX}$ & RFA & RFA & RFA & RFA & DN & 72.61 & 195276.85 \\
\hline 97 & RFA & RFA & TX & TX & $\mathrm{TX}$ & $\mathrm{TX}$ & Sorafenib & 65.50 & 90744.87 \\
\hline 98 & RFA & RFA & TX & $\mathrm{TX}$ & $\mathrm{TX}$ & TX & DN & 65.47 & 90704.34 \\
\hline 99 & RFA & RFA & $\mathrm{TX}$ & $\mathrm{TX}$ & $\mathrm{TX}$ & RFA & Sorafenib & 65.42 & 89628.53 \\
\hline 100 & RFA & RFA & $\mathrm{TX}$ & $\mathrm{TX}$ & $\mathrm{TX}$ & RFA & $\mathrm{DN}$ & 65.39 & 89588.00 \\
\hline 101 & RFA & RFA & $\mathrm{TX}$ & TX & RFA & $\mathrm{TX}$ & Sorafenib & 65.27 & 87395.85 \\
\hline 102 & RFA & RFA & $\mathrm{TX}$ & $\mathrm{TX}$ & RFA & TX & DN & 65.24 & 87355.32 \\
\hline 103 & RFA & RFA & $\mathrm{TX}$ & $\mathrm{TX}$ & RFA & RFA & Sorafenib & 65.19 & 86279.51 \\
\hline 104 & RFA & RFA & $\mathrm{TX}$ & $\mathrm{TX}$ & RFA & RFA & DN & 65.16 & 86238.97 \\
\hline 105 & RFA & RFA & $\mathrm{TX}$ & RFA & $\mathrm{TX}$ & $\mathrm{TX}$ & Sorafenib & 64.73 & 79555.51 \\
\hline 106 & RFA & RFA & $\mathrm{TX}$ & RFA & $\mathrm{TX}$ & $\mathrm{TX}$ & DN & 64.71 & 79514.97 \\
\hline 107 & RFA & RFA & TX & RFA & $\mathrm{TX}$ & RFA & Sorafenib & 64.66 & 78439.17 \\
\hline 108 & RFA & RFA & $\mathrm{TX}$ & RFA & $\mathrm{TX}$ & RFA & DN & 64.63 & 78398.63 \\
\hline 109 & RFA & RFA & $\mathrm{TX}$ & RFA & RFA & TX & Sorafenib & 64.50 & 76206.49 \\
\hline 110 & RFA & RFA & TX & RFA & RFA & $\mathrm{TX}$ & DN & 64.48 & 76165.95 \\
\hline 111 & RFA & RFA & TX & RFA & RFA & RFA & Sorafenib & 64.43 & 75090.15 \\
\hline 112 & RFA & RFA & TX & RFA & RFA & RFA & DN & 64.40 & 75049.61 \\
\hline 113 & RFA & RFA & RFA & TX & $\mathrm{TX}$ & TX & Sorafenib & 61.72 & 35369.20 \\
\hline 114 & RFA & RFA & RFA & $\mathrm{TX}$ & $\mathrm{TX}$ & TX & DN & 61.69 & 35328.67 \\
\hline 115 & RFA & RFA & RFA & $\mathrm{TX}$ & $\mathrm{TX}$ & RFA & Sorafenib & 61.64 & 34252.86 \\
\hline 116 & RFA & RFA & RFA & $\mathrm{TX}$ & $\mathrm{TX}$ & RFA & DN & 61.61 & 34212.33 \\
\hline 117 & RFA & RFA & RFA & $\mathrm{TX}$ & RFA & TX & Sorafenib & 61.49 & 32020.18 \\
\hline 118 & RFA & RFA & RFA & $\mathrm{TX}$ & RFA & $\mathrm{TX}$ & DN & 61.46 & 31979.65 \\
\hline 119 & RFA & RFA & RFA & TX & RFA & RFA & Sorafenib & 61.41 & 30903.84 \\
\hline 120 & RFA & RFA & RFA & TX & RFA & RFA & DN & 61.39 & 30863.31 \\
\hline 121 & RFA & RFA & RFA & RFA & $\mathrm{TX}$ & $\mathrm{TX}$ & Sorafenib & 60.95 & 24179.84 \\
\hline 122 & RFA & RFA & RFA & RFA & $\mathrm{TX}$ & TX & DN & 60.93 & 24139.31 \\
\hline 123 & RFA & RFA & RFA & RFA & $\mathrm{TX}$ & RFA & Sorafenib & 60.88 & 23063.50 \\
\hline 124 & RFA & RFA & RFA & RFA & $\mathrm{TX}$ & RFA & DN & 60.85 & 23022.97 \\
\hline 125 & RFA & RFA & RFA & RFA & RFA & TX & Sorafenib & 60.72 & 20830.82 \\
\hline 126 & RFA & RFA & RFA & RFA & RFA & $\mathrm{TX}$ & DN & 60.70 & 20790.29 \\
\hline 127 & RFA & RFA & RFA & RFA & RFA & RFA & Sorafenib & 60.65 & 19714.48 \\
\hline 128 & RFA & RFA & RFA & RFA & RFA & RFA & DN & 60.62 & 19673.95 \\
\hline 129 & $\mathrm{DN}$ & $\mathrm{TX}$ & $\mathrm{TX}$ & $\mathrm{TX}$ & $\mathrm{TX}$ & $\mathrm{TX}$ & Sorafenib & 76.25 & 260900.48 \\
\hline
\end{tabular}




\begin{tabular}{|c|c|c|c|c|c|c|c|c|c|}
\hline 130 & $\mathrm{DN}$ & $\mathrm{TX}$ & $\mathrm{TX}$ & $\mathrm{TX}$ & $\mathrm{TX}$ & $\mathrm{TX}$ & $\mathrm{DN}$ & 76.22 & 260866.94 \\
\hline 131 & $\mathrm{DN}$ & $\mathrm{TX}$ & $\mathrm{TX}$ & $\mathrm{TX}$ & $\mathrm{TX}$ & DN & Sorafenib & 75.95 & 260885.16 \\
\hline 132 & $\mathrm{DN}$ & $\mathrm{TX}$ & $\mathrm{TX}$ & $\mathrm{TX}$ & $\mathrm{TX}$ & $\mathrm{DN}$ & $\mathrm{DN}$ & 75.92 & 260851.63 \\
\hline 133 & $\mathrm{DN}$ & $\mathrm{TX}$ & $\mathrm{TX}$ & $\mathrm{TX}$ & $\mathrm{DN}$ & $\mathrm{TX}$ & Sorafenib & 74.95 & 256466.24 \\
\hline 134 & $\mathrm{DN}$ & $\mathrm{TX}$ & $\mathrm{TX}$ & $\mathrm{TX}$ & $\mathrm{DN}$ & $\mathrm{TX}$ & DN & 74.93 & 256432.70 \\
\hline 135 & $\mathrm{DN}$ & $\mathrm{TX}$ & $\mathrm{TX}$ & $\mathrm{TX}$ & $\mathrm{DN}$ & $\mathrm{DN}$ & Sorafenib & 74.65 & 256450.92 \\
\hline 136 & $\mathrm{DN}$ & $\mathrm{TX}$ & $\mathrm{TX}$ & $\mathrm{TX}$ & $\mathrm{DN}$ & $\mathrm{DN}$ & DN & 74.63 & 256417.39 \\
\hline 137 & $\mathrm{DN}$ & $\mathrm{TX}$ & $\mathrm{TX}$ & $\mathrm{DN}$ & $\mathrm{TX}$ & $\mathrm{TX}$ & Sorafenib & 72.25 & 247237.48 \\
\hline 138 & $\mathrm{DN}$ & $\mathrm{TX}$ & $\mathrm{TX}$ & $\mathrm{DN}$ & $\mathrm{TX}$ & $\mathrm{TX}$ & $\mathrm{DN}$ & 72.23 & 247203.94 \\
\hline 139 & $\mathrm{DN}$ & $\mathrm{TX}$ & $\mathrm{TX}$ & $\mathrm{DN}$ & $\mathrm{TX}$ & $\mathrm{DN}$ & Sorafenib & 71.95 & 247222.16 \\
\hline 140 & $\mathrm{DN}$ & $\mathrm{TX}$ & $\mathrm{TX}$ & $\mathrm{DN}$ & $\mathrm{TX}$ & $\mathrm{DN}$ & $\mathrm{DN}$ & 71.93 & 247188.62 \\
\hline 141 & $\mathrm{DN}$ & $\mathrm{TX}$ & $\mathrm{TX}$ & $\mathrm{DN}$ & $\mathrm{DN}$ & $\mathrm{TX}$ & Sorafenib & 70.96 & 242803.24 \\
\hline 142 & $\mathrm{DN}$ & $\mathrm{TX}$ & $\mathrm{TX}$ & $\mathrm{DN}$ & $\mathrm{DN}$ & $\mathrm{TX}$ & DN & 70.93 & 242769.70 \\
\hline 143 & $\mathrm{DN}$ & $\mathrm{TX}$ & $\mathrm{TX}$ & $\mathrm{DN}$ & $\mathrm{DN}$ & $\mathrm{DN}$ & Sorafenib & 70.66 & 242787.92 \\
\hline 144 & $\mathrm{DN}$ & $\mathrm{TX}$ & $\mathrm{TX}$ & $\mathrm{DN}$ & $\mathrm{DN}$ & $\mathrm{DN}$ & $\mathrm{DN}$ & 70.63 & 242754.38 \\
\hline 145 & $\mathrm{DN}$ & $\mathrm{TX}$ & $\mathrm{DN}$ & $\mathrm{TX}$ & $\mathrm{TX}$ & $\mathrm{TX}$ & Sorafenib & 55.73 & 190700.92 \\
\hline 146 & $\mathrm{DN}$ & $\mathrm{TX}$ & $\mathrm{DN}$ & $\mathrm{TX}$ & $\mathrm{TX}$ & $\mathrm{TX}$ & DN & 55.70 & 190667.38 \\
\hline 147 & $\mathrm{DN}$ & $\mathrm{TX}$ & $\mathrm{DN}$ & $\mathrm{TX}$ & $\mathrm{TX}$ & $\mathrm{DN}$ & Sorafenib & 55.43 & 190685.60 \\
\hline 148 & $\mathrm{DN}$ & $\mathrm{TX}$ & $\mathrm{DN}$ & $\mathrm{TX}$ & $\mathrm{TX}$ & $\mathrm{DN}$ & DN & 55.40 & 190652.06 \\
\hline 149 & $\mathrm{DN}$ & $\mathrm{TX}$ & $\mathrm{DN}$ & $\mathrm{TX}$ & $\mathrm{DN}$ & $\mathrm{TX}$ & Sorafenib & 54.43 & 186266.68 \\
\hline 150 & $\mathrm{DN}$ & $\mathrm{TX}$ & $\mathrm{DN}$ & $\mathrm{TX}$ & $\mathrm{DN}$ & $\mathrm{TX}$ & DN & 54.41 & 186233.14 \\
\hline 151 & $\mathrm{DN}$ & $\mathrm{TX}$ & $\mathrm{DN}$ & $\mathrm{TX}$ & $\mathrm{DN}$ & $\mathrm{DN}$ & Sorafenib & 54.13 & 186251.36 \\
\hline 152 & $\mathrm{DN}$ & $\mathrm{TX}$ & $\mathrm{DN}$ & $\mathrm{TX}$ & $\mathrm{DN}$ & $\mathrm{DN}$ & $\mathrm{DN}$ & 54.11 & 186217.82 \\
\hline 153 & $\mathrm{DN}$ & $\mathrm{TX}$ & $\mathrm{DN}$ & $\mathrm{DN}$ & $\mathrm{TX}$ & $\mathrm{TX}$ & Sorafenib & 51.73 & 177037.91 \\
\hline 154 & $\mathrm{DN}$ & $\mathrm{TX}$ & $\mathrm{DN}$ & $\mathrm{DN}$ & $\mathrm{TX}$ & $\mathrm{TX}$ & $\mathrm{DN}$ & 51.71 & 177004.38 \\
\hline 155 & $\mathrm{DN}$ & $\mathrm{TX}$ & $\mathrm{DN}$ & $\mathrm{DN}$ & $\mathrm{TX}$ & DN & Sorafenib & 51.44 & 177022.60 \\
\hline 156 & $\mathrm{DN}$ & $\mathrm{TX}$ & $\mathrm{DN}$ & $\mathrm{DN}$ & $\mathrm{TX}$ & $\mathrm{DN}$ & DN & 51.41 & 176989.06 \\
\hline 157 & $\mathrm{DN}$ & $\mathrm{TX}$ & $\mathrm{DN}$ & $\mathrm{DN}$ & $\mathrm{DN}$ & $\mathrm{TX}$ & Sorafenib & 50.44 & 172603.67 \\
\hline 158 & $\mathrm{DN}$ & $\mathrm{TX}$ & $\mathrm{DN}$ & $\mathrm{DN}$ & $\mathrm{DN}$ & $\mathrm{TX}$ & DN & 50.41 & 172570.14 \\
\hline 159 & $\mathrm{DN}$ & $\mathrm{TX}$ & $\mathrm{DN}$ & $\mathrm{DN}$ & $\mathrm{DN}$ & $\mathrm{DN}$ & Sorafenib & 50.14 & 172588.36 \\
\hline 160 & $\mathrm{DN}$ & $\mathrm{TX}$ & $\mathrm{DN}$ & $\mathrm{DN}$ & $\mathrm{DN}$ & $\mathrm{DN}$ & $\mathrm{DN}$ & 50.11 & 172554.82 \\
\hline 161 & $\mathrm{DN}$ & $\mathrm{DN}$ & $\mathrm{TX}$ & $\mathrm{TX}$ & $\mathrm{TX}$ & $\mathrm{TX}$ & Sorafenib & 26.13 & 89433.96 \\
\hline 162 & $\mathrm{DN}$ & $\mathrm{DN}$ & $\mathrm{TX}$ & $\mathrm{TX}$ & $\mathrm{TX}$ & $\mathrm{TX}$ & DN & 26.11 & 89400.43 \\
\hline 163 & $\mathrm{DN}$ & $\mathrm{DN}$ & $\mathrm{TX}$ & $\mathrm{TX}$ & $\mathrm{TX}$ & $\mathrm{DN}$ & Sorafenib & 25.83 & 89418.64 \\
\hline 164 & $\mathrm{DN}$ & $\mathrm{DN}$ & $\mathrm{TX}$ & $\mathrm{TX}$ & $\mathrm{TX}$ & $\mathrm{DN}$ & DN & 25.81 & 89385.11 \\
\hline 165 & $\mathrm{DN}$ & $\mathrm{DN}$ & $\mathrm{TX}$ & $\mathrm{TX}$ & $\mathrm{DN}$ & $\mathrm{TX}$ & Sorafenib & 24.83 & 84999.72 \\
\hline 166 & $\mathrm{DN}$ & $\mathrm{DN}$ & $\mathrm{TX}$ & $\mathrm{TX}$ & $\mathrm{DN}$ & $\mathrm{TX}$ & DN & 24.81 & 84966.19 \\
\hline 167 & $\mathrm{DN}$ & $\mathrm{DN}$ & $\mathrm{TX}$ & $\mathrm{TX}$ & $\mathrm{DN}$ & DN & Sorafenib & 24.53 & 84984.40 \\
\hline 168 & $\mathrm{DN}$ & $\mathrm{DN}$ & $\mathrm{TX}$ & $\mathrm{TX}$ & $\mathrm{DN}$ & $\mathrm{DN}$ & $\mathrm{DN}$ & 24.51 & 84950.87 \\
\hline 169 & $\mathrm{DN}$ & $\mathrm{DN}$ & $\mathrm{TX}$ & $\mathrm{DN}$ & $\mathrm{TX}$ & $\mathrm{TX}$ & Sorafenib & 22.14 & 75770.96 \\
\hline 170 & $\mathrm{DN}$ & $\mathrm{DN}$ & $\mathrm{TX}$ & $\mathrm{DN}$ & $\mathrm{TX}$ & $\mathrm{TX}$ & DN & 22.11 & 75737.42 \\
\hline 171 & $\mathrm{DN}$ & $\mathrm{DN}$ & $\mathrm{TX}$ & $\mathrm{DN}$ & $\mathrm{TX}$ & $\mathrm{DN}$ & Sorafenib & 21.84 & 75755.64 \\
\hline 172 & $\mathrm{DN}$ & $\mathrm{DN}$ & $\mathrm{TX}$ & $\mathrm{DN}$ & $\mathrm{TX}$ & $\mathrm{DN}$ & DN & 21.81 & 75722.11 \\
\hline 173 & $\mathrm{DN}$ & $\mathrm{DN}$ & $\mathrm{TX}$ & $\mathrm{DN}$ & $\mathrm{DN}$ & $\mathrm{TX}$ & Sorafenib & 20.84 & 71336.72 \\
\hline 174 & $\mathrm{DN}$ & $\mathrm{DN}$ & $\mathrm{TX}$ & $\mathrm{DN}$ & $\mathrm{DN}$ & $\mathrm{TX}$ & DN & 20.82 & 71303.18 \\
\hline 175 & $\mathrm{DN}$ & $\mathrm{DN}$ & $\mathrm{TX}$ & $\mathrm{DN}$ & $\mathrm{DN}$ & DN & Sorafenib & 20.54 & 71321.40 \\
\hline
\end{tabular}




\begin{tabular}{|c|c|c|c|c|c|c|c|c|c|}
\hline 176 & $\mathrm{DN}$ & $\mathrm{DN}$ & $\mathrm{TX}$ & $\mathrm{DN}$ & $\mathrm{DN}$ & $\mathrm{DN}$ & $\mathrm{DN}$ & 20.52 & 71287.87 \\
\hline 177 & $\mathrm{DN}$ & $\mathrm{DN}$ & $\mathrm{DN}$ & $\mathrm{TX}$ & $\mathrm{TX}$ & $\mathrm{TX}$ & Sorafenib & 5.61 & 19234.40 \\
\hline 178 & $\mathrm{DN}$ & $\mathrm{DN}$ & $\mathrm{DN}$ & $\mathrm{TX}$ & $\mathrm{TX}$ & $\mathrm{TX}$ & $\mathrm{DN}$ & 5.59 & 19200.86 \\
\hline 179 & $\mathrm{DN}$ & $\mathrm{DN}$ & $\mathrm{DN}$ & TX & $\mathrm{TX}$ & $\mathrm{DN}$ & Sorafenib & 5.31 & 19219.08 \\
\hline 180 & $\mathrm{DN}$ & $\mathrm{DN}$ & $\mathrm{DN}$ & TX & $\mathrm{TX}$ & $\mathrm{DN}$ & $\mathrm{DN}$ & 5.29 & 19185.55 \\
\hline 181 & $\mathrm{DN}$ & $\mathrm{DN}$ & $\mathrm{DN}$ & TX & $\mathrm{DN}$ & $\mathrm{TX}$ & Sorafenib & 4.32 & 14800.16 \\
\hline 182 & $\mathrm{DN}$ & $\mathrm{DN}$ & $\mathrm{DN}$ & TX & $\mathrm{DN}$ & $\mathrm{TX}$ & $\mathrm{DN}$ & 4.29 & 14766.62 \\
\hline 183 & $\mathrm{DN}$ & $\mathrm{DN}$ & DN & TX & $\mathrm{DN}$ & $\mathrm{DN}$ & Sorafenib & 4.02 & 14784.84 \\
\hline 184 & $\mathrm{DN}$ & $\mathrm{DN}$ & $\mathrm{DN}$ & $\mathrm{TX}$ & $\mathrm{DN}$ & $\mathrm{DN}$ & $\mathrm{DN}$ & 3.99 & 14751.31 \\
\hline 185 & $\mathrm{DN}$ & $\mathrm{DN}$ & $\mathrm{DN}$ & $\mathrm{DN}$ & $\mathrm{TX}$ & $\mathrm{TX}$ & Sorafenib & 1.62 & 5571.40 \\
\hline 186 & $\mathrm{DN}$ & $\mathrm{DN}$ & $\mathrm{DN}$ & $\mathrm{DN}$ & $\mathrm{TX}$ & $\mathrm{TX}$ & $\mathrm{DN}$ & 1.60 & 5537.86 \\
\hline 187 & $\mathrm{DN}$ & $\mathrm{DN}$ & $\mathrm{DN}$ & $\mathrm{DN}$ & $\mathrm{TX}$ & DN & Sorafenib & 1.32 & 5556.08 \\
\hline 188 & $\mathrm{DN}$ & $\mathrm{DN}$ & $\mathrm{DN}$ & $\mathrm{DN}$ & $\mathrm{TX}$ & $\mathrm{DN}$ & DN & 1.30 & 5522.54 \\
\hline 189 & $\mathrm{DN}$ & $\mathrm{DN}$ & DN & $\mathrm{DN}$ & $\mathrm{DN}$ & $\mathrm{TX}$ & Sorafenib & 0.32 & 1137.16 \\
\hline 190 & $\mathrm{DN}$ & $\mathrm{DN}$ & $\mathrm{DN}$ & $\mathrm{DN}$ & $\mathrm{DN}$ & $\mathrm{TX}$ & $\mathrm{DN}$ & 0.30 & 1103.62 \\
\hline 191 & $\mathrm{DN}$ & $\mathrm{DN}$ & $\mathrm{DN}$ & $\mathrm{DN}$ & $\mathrm{DN}$ & $\mathrm{DN}$ & Sorafenib & 0.02 & 1121.84 \\
\hline 192 & $\mathrm{DN}$ & $\mathrm{DN}$ & $\mathrm{DN}$ & $\mathrm{DN}$ & $\mathrm{DN}$ & $\mathrm{DN}$ & $\mathrm{DN}$ & 0.00 & 1088.30 \\
\hline
\end{tabular}

\section{Initial Calculated MELD [10,15)}

\begin{tabular}{|c|c|c|c|c|c|c|c|c|c|c|}
\hline & & & & & & & & & hage Vector & \\
\hline & $\begin{array}{c}\text { Initial } \\
\text { Decision }\end{array}$ & M1 & M2 & M3 & M4 & M5 & Drop & Lives & Cost & $\mathrm{P}-\mathrm{O} ?$ \\
\hline 1 & TACE & $\mathrm{TX}$ & $\mathrm{TX}$ & $\mathrm{TX}$ & $\mathrm{TX}$ & $\mathrm{TX}$ & Sorafenib & 77.72 & 271121.30 & \\
\hline 2 & TACE & $\mathrm{TX}$ & $\mathrm{TX}$ & $\mathrm{TX}$ & $\mathrm{TX}$ & $\mathrm{TX}$ & $\mathrm{DN}$ & 77.68 & 271062.86 & \\
\hline 3 & TACE & $\mathrm{TX}$ & TX & TX & $\mathrm{TX}$ & TACE & Sorafenib & 77.15 & 266326.45 & $\mathrm{X}$ \\
\hline 4 & TACE & $\mathrm{TX}$ & TX & $\mathrm{TX}$ & $\mathrm{TX}$ & TACE & $\mathrm{DN}$ & 77.12 & 266268.00 & $\mathrm{X}$ \\
\hline 5 & TACE & $\mathrm{TX}$ & $\mathrm{TX}$ & $\mathrm{TX}$ & TACE & $\mathrm{TX}$ & Sorafenib & 76.24 & 258537.98 & $\mathrm{X}$ \\
\hline 6 & TACE & $\mathrm{TX}$ & TX & $\mathrm{TX}$ & TACE & $\mathrm{TX}$ & $\mathrm{DN}$ & 76.20 & 258479.54 & $\mathrm{X}$ \\
\hline 7 & TACE & $\mathrm{TX}$ & $\mathrm{TX}$ & $\mathrm{TX}$ & TACE & TACE & Sorafenib & 75.68 & 253743.13 & $\mathrm{X}$ \\
\hline 8 & TACE & $\mathrm{TX}$ & $\mathrm{TX}$ & $\mathrm{TX}$ & TACE & TACE & $\mathrm{DN}$ & 75.64 & 253684.68 & \\
\hline 9 & TACE & $\mathrm{TX}$ & $\mathrm{TX}$ & TACE & $\mathrm{TX}$ & $\mathrm{TX}$ & Sorafenib & 72.04 & 222766.85 & $\mathrm{X}$ \\
\hline 10 & TACE & $\mathrm{TX}$ & $\mathrm{TX}$ & TACE & $\mathrm{TX}$ & $\mathrm{TX}$ & $\mathrm{DN}$ & 72.00 & 222708.40 & $\mathrm{X}$ \\
\hline 11 & TACE & $\mathrm{TX}$ & $\mathrm{TX}$ & TACE & $\mathrm{TX}$ & TACE & Sorafenib & 71.48 & 217971.99 & $\mathrm{X}$ \\
\hline 12 & TACE & $\mathrm{TX}$ & $\mathrm{TX}$ & TACE & $\mathrm{TX}$ & TACE & $\mathrm{DN}$ & 71.44 & 217913.55 & $\mathrm{X}$ \\
\hline 13 & TACE & $\mathrm{TX}$ & $\mathrm{TX}$ & TACE & TACE & $\mathrm{TX}$ & Sorafenib & 70.56 & 210183.52 & $\mathrm{X}$ \\
\hline 14 & TACE & $\mathrm{TX}$ & $\mathrm{TX}$ & TACE & TACE & $\mathrm{TX}$ & $\mathrm{DN}$ & 70.53 & 210125.08 & $\mathrm{X}$ \\
\hline 15 & TACE & $\mathrm{TX}$ & $\mathrm{TX}$ & TACE & TACE & TACE & Sorafenib & 70.00 & 205388.67 & $\mathrm{X}$ \\
\hline 16 & TACE & $\mathrm{TX}$ & $\mathrm{TX}$ & TACE & TACE & TACE & $\mathrm{DN}$ & 69.97 & 205330.23 & $\mathrm{X}$ \\
\hline 17 & TACE & $\mathrm{TX}$ & TACE & $\mathrm{TX}$ & $\mathrm{TX}$ & $\mathrm{TX}$ & Sorafenib & 60.77 & 126742.91 & $\mathrm{X}$ \\
\hline 18 & TACE & $\mathrm{TX}$ & TACE & $\mathrm{TX}$ & $\mathrm{TX}$ & $\mathrm{TX}$ & DN & 60.74 & 126684.47 & $\mathrm{X}$ \\
\hline 19 & TACE & $\mathrm{TX}$ & TACE & $\mathrm{TX}$ & $\mathrm{TX}$ & TACE & Sorafenib & 60.21 & 121948.06 & $\mathrm{X}$ \\
\hline 20 & TACE & $\mathrm{TX}$ & TACE & $\mathrm{TX}$ & $\mathrm{TX}$ & TACE & $\mathrm{DN}$ & 60.17 & 121889.61 & $\mathrm{X}$ \\
\hline
\end{tabular}




\begin{tabular}{|c|c|c|c|c|c|c|c|c|c|}
\hline 21 & TACE & $\mathrm{TX}$ & TACE & $\mathrm{TX}$ & TACE & $\mathrm{TX}$ & Sorafenib & 59.30 & 114159.59 \\
\hline 22 & TACE & $\mathrm{TX}$ & TACE & $\mathrm{TX}$ & TACE & $\mathrm{TX}$ & DN & 59.26 & 114101.14 \\
\hline 23 & TACE & $\mathrm{TX}$ & TACE & $\mathrm{TX}$ & TACE & TACE & Sorafenib & 58.73 & 109364.73 \\
\hline 24 & TACE & $\mathrm{TX}$ & TACE & $\mathrm{TX}$ & TACE & TACE & DN & 58.70 & 109306.29 \\
\hline 25 & TACE & $\mathrm{TX}$ & TACE & TACE & $\mathrm{TX}$ & $\mathrm{TX}$ & Sorafenib & 55.10 & 78388.45 \\
\hline 26 & TACE & $\mathrm{TX}$ & TACE & TACE & $\mathrm{TX}$ & $\mathrm{TX}$ & $\mathrm{DN}$ & 55.06 & 78330.01 \\
\hline 27 & TACE & $\mathrm{TX}$ & TACE & TACE & $\mathrm{TX}$ & TACE & Sorafenib & 54.54 & 73593.60 \\
\hline 28 & TACE & $\mathrm{TX}$ & TACE & TACE & $\mathrm{TX}$ & TACE & $\mathrm{DN}$ & 54.50 & 73535.15 \\
\hline 29 & TACE & $\mathrm{TX}$ & TACE & TACE & TACE & $\mathrm{TX}$ & Sorafenib & 53.62 & 65805.13 \\
\hline 30 & TACE & $\mathrm{TX}$ & TACE & TACE & TACE & $\mathrm{TX}$ & DN & 53.59 & 65746.69 \\
\hline 31 & TACE & $\mathrm{TX}$ & TACE & TACE & TACE & TACE & Sorafenib & 53.06 & 61010.28 \\
\hline 32 & TACE & $\mathrm{TX}$ & TACE & TACE & TACE & TACE & DN & 53.02 & 60951.83 \\
\hline 33 & TACE & TACE & $\mathrm{TX}$ & $\mathrm{TX}$ & $\mathrm{TX}$ & $\mathrm{TX}$ & Sorafenib & 73.97 & 239231.72 \\
\hline 34 & TACE & TACE & $\mathrm{TX}$ & $\mathrm{TX}$ & $\mathrm{TX}$ & $\mathrm{TX}$ & DN & 73.94 & 239173.27 \\
\hline 35 & TACE & TACE & $\mathrm{TX}$ & $\mathrm{TX}$ & $\mathrm{TX}$ & TACE & Sorafenib & 73.41 & 234436.86 \\
\hline 36 & TACE & TACE & $\mathrm{TX}$ & $\mathrm{TX}$ & $\mathrm{TX}$ & TACE & $\mathrm{DN}$ & 73.37 & 234378.42 \\
\hline 37 & TACE & TACE & $\mathrm{TX}$ & $\mathrm{TX}$ & TACE & $\mathrm{TX}$ & Sorafenib & 72.50 & 226648.40 \\
\hline 38 & TACE & TACE & $\mathrm{TX}$ & $\mathrm{TX}$ & TACE & $\mathrm{TX}$ & $\mathrm{DN}$ & 72.46 & 226589.95 \\
\hline 39 & TACE & TACE & $\mathrm{TX}$ & $\mathrm{TX}$ & TACE & TACE & Sorafenib & 71.93 & 221853.54 \\
\hline 40 & TACE & TACE & $\mathrm{TX}$ & $\mathrm{TX}$ & TACE & TACE & DN & 71.90 & 221795.10 \\
\hline 41 & TACE & TACE & $\mathrm{TX}$ & TACE & $\mathrm{TX}$ & $\mathrm{TX}$ & Sorafenib & 68.30 & 190877.26 \\
\hline 42 & TACE & TACE & $\mathrm{TX}$ & TACE & $\mathrm{TX}$ & $\mathrm{TX}$ & $\mathrm{DN}$ & 68.26 & 190818.81 \\
\hline 43 & TACE & TACE & $\mathrm{TX}$ & TACE & $\mathrm{TX}$ & TACE & Sorafenib & 67.74 & 186082.40 \\
\hline 44 & TACE & TACE & $\mathrm{TX}$ & TACE & $\mathrm{TX}$ & TACE & $\mathrm{DN}$ & 67.70 & 186023.96 \\
\hline 45 & TACE & TACE & $\mathrm{TX}$ & TACE & TACE & $\mathrm{TX}$ & Sorafenib & 66.82 & 178293.94 \\
\hline 46 & TACE & TACE & $\mathrm{TX}$ & TACE & TACE & $\mathrm{TX}$ & $\mathrm{DN}$ & 66.79 & 178235.49 \\
\hline 47 & TACE & TACE & TX & TACE & TACE & TACE & Sorafenib & 66.26 & 173499.08 \\
\hline 48 & TACE & TACE & $\mathrm{TX}$ & TACE & TACE & TACE & $\mathrm{DN}$ & 66.22 & 173440.64 \\
\hline 49 & TACE & TACE & TACE & $\mathrm{TX}$ & $\mathrm{TX}$ & $\mathrm{TX}$ & Sorafenib & 57.03 & 94853.32 \\
\hline 50 & TACE & TACE & TACE & $\mathrm{TX}$ & $\mathrm{TX}$ & $\mathrm{TX}$ & DN & 56.99 & 94794.88 \\
\hline 51 & TACE & TACE & TACE & $\mathrm{TX}$ & $\mathrm{TX}$ & TACE & Sorafenib & 56.47 & 90058.47 \\
\hline 52 & TACE & TACE & TACE & $\mathrm{TX}$ & $\mathrm{TX}$ & TACE & DN & 56.43 & 90000.02 \\
\hline 53 & TACE & TACE & TACE & $\mathrm{TX}$ & TACE & $\mathrm{TX}$ & Sorafenib & 55.55 & 82270.00 \\
\hline 54 & TACE & TACE & TACE & $\mathrm{TX}$ & TACE & $\mathrm{TX}$ & $\mathrm{DN}$ & 55.52 & 82211.56 \\
\hline 55 & TACE & TACE & TACE & $\mathrm{TX}$ & TACE & TACE & Sorafenib & 54.99 & 77475.15 \\
\hline 56 & TACE & TACE & TACE & $\mathrm{TX}$ & TACE & TACE & $\mathrm{DN}$ & 54.95 & 77416.70 \\
\hline 57 & TACE & TACE & TACE & TACE & $\mathrm{TX}$ & $\mathrm{TX}$ & Sorafenib & 51.36 & 46498.87 \\
\hline 58 & TACE & TACE & TACE & TACE & $\mathrm{TX}$ & $\mathrm{TX}$ & DN & 51.32 & 46440.42 \\
\hline 59 & TACE & TACE & TACE & TACE & $\mathrm{TX}$ & TACE & Sorafenib & 50.79 & 41704.01 \\
\hline 60 & TACE & TACE & TACE & TACE & $\mathrm{TX}$ & TACE & DN & 50.76 & 41645.57 \\
\hline 61 & TACE & TACE & TACE & TACE & TACE & $\mathrm{TX}$ & Sorafenib & 49.88 & 33915.54 \\
\hline 62 & TACE & TACE & TACE & TACE & TACE & $\mathrm{TX}$ & DN & 49.84 & 33857.10 \\
\hline 63 & TACE & TACE & TACE & TACE & TACE & TACE & Sorafenib & 49.32 & 29120.69 \\
\hline 64 & TACE & TACE & TACE & TACE & TACE & TACE & DN & 49.28 & 29062.24 \\
\hline 65 & RFA & $\mathrm{TX}$ & $\mathrm{TX}$ & $\mathrm{TX}$ & $\mathrm{TX}$ & $\mathrm{TX}$ & Sorafenib & 77.48 & 266347.78 \\
\hline & RFA & TX & X & TX & TX & $x$ & DN & 77.46 & 266307.25 \\
\hline
\end{tabular}




\begin{tabular}{|c|c|c|c|c|c|c|c|c|c|}
\hline 67 & RFA & $\mathrm{TX}$ & $\mathrm{TX}$ & $\mathrm{TX}$ & $\mathrm{TX}$ & RFA & Sorafenib & 77.33 & 264167.02 \\
\hline 68 & RFA & $\mathrm{TX}$ & $\mathrm{TX}$ & $\mathrm{TX}$ & $\mathrm{TX}$ & RFA & DN & 77.31 & 264126.49 \\
\hline 69 & RFA & $\mathrm{TX}$ & $\mathrm{TX}$ & $\mathrm{TX}$ & RFA & $\mathrm{TX}$ & Sorafenib & 76.77 & 255963.22 \\
\hline 70 & RFA & $\mathrm{TX}$ & $\mathrm{TX}$ & $\mathrm{TX}$ & RFA & $\mathrm{TX}$ & DN & 76.75 & 255922.69 \\
\hline 71 & RFA & $\mathrm{TX}$ & $\mathrm{TX}$ & $\mathrm{TX}$ & RFA & RFA & Sorafenib & 76.62 & 253782.46 \\
\hline 72 & RFA & $\mathrm{TX}$ & $\mathrm{TX}$ & $\mathrm{TX}$ & RFA & RFA & DN & 76.60 & 253741.93 \\
\hline 73 & RFA & $\mathrm{TX}$ & $\mathrm{TX}$ & RFA & $\mathrm{TX}$ & $\mathrm{TX}$ & Sorafenib & 74.20 & 218215.34 \\
\hline 74 & RFA & $\mathrm{TX}$ & $\mathrm{TX}$ & RFA & $\mathrm{TX}$ & $\mathrm{TX}$ & DN & 74.17 & 218174.81 \\
\hline 75 & RFA & $\mathrm{TX}$ & $\mathrm{TX}$ & RFA & $\mathrm{TX}$ & RFA & Sorafenib & 74.05 & 216034.59 \\
\hline 76 & RFA & $\mathrm{TX}$ & TX & RFA & $\mathrm{TX}$ & RFA & $\mathrm{DN}$ & 74.02 & 215994.05 \\
\hline 77 & RFA & $\mathrm{TX}$ & TX & RFA & RFA & $\mathrm{TX}$ & Sorafenib & 73.49 & 207830.78 \\
\hline 78 & RFA & $\mathrm{TX}$ & $\mathrm{TX}$ & RFA & RFA & $\mathrm{TX}$ & DN & 73.46 & 207790.25 \\
\hline 79 & RFA & $\mathrm{TX}$ & $\mathrm{TX}$ & RFA & RFA & RFA & Sorafenib & 73.34 & 205650.03 \\
\hline 80 & RFA & $\mathrm{TX}$ & $\mathrm{TX}$ & RFA & RFA & RFA & DN & 73.31 & 205609.49 \\
\hline 81 & RFA & $\mathrm{TX}$ & RFA & $\mathrm{TX}$ & $\mathrm{TX}$ & $\mathrm{TX}$ & Sorafenib & 66.73 & 108839.97 \\
\hline 82 & RFA & $\mathrm{TX}$ & RFA & $\mathrm{TX}$ & $\mathrm{TX}$ & $\mathrm{TX}$ & DN & 66.70 & 108799.43 \\
\hline 83 & RFA & $\mathrm{TX}$ & RFA & $\mathrm{TX}$ & $\mathrm{TX}$ & RFA & Sorafenib & 66.58 & 106659.21 \\
\hline 84 & RFA & $\mathrm{TX}$ & RFA & $\mathrm{TX}$ & $\mathrm{TX}$ & RFA & DN & 66.56 & 106618.67 \\
\hline 85 & RFA & $\mathrm{TX}$ & RFA & $\mathrm{TX}$ & RFA & $\mathrm{TX}$ & Sorafenib & 66.02 & 98455.41 \\
\hline 86 & RFA & $\mathrm{TX}$ & RFA & $\mathrm{TX}$ & RFA & $\mathrm{TX}$ & DN & 66.00 & 98414.87 \\
\hline 87 & RFA & $\mathrm{TX}$ & RFA & $\mathrm{TX}$ & RFA & RFA & Sorafenib & 65.87 & 96274.65 \\
\hline 88 & RFA & $\mathrm{TX}$ & RFA & $\mathrm{TX}$ & RFA & RFA & $\mathrm{DN}$ & 65.85 & 96234.11 \\
\hline 89 & RFA & $\mathrm{TX}$ & RFA & RFA & $\mathrm{TX}$ & $\mathrm{TX}$ & Sorafenib & 63.45 & 60707.53 \\
\hline 90 & RFA & $\mathrm{TX}$ & RFA & RFA & $\mathrm{TX}$ & $\mathrm{TX}$ & DN & 63.42 & 60667.00 \\
\hline 91 & RFA & $\mathrm{TX}$ & RFA & RFA & $\mathrm{TX}$ & RFA & Sorafenib & 63.30 & 58526.77 \\
\hline 92 & RFA & $\mathrm{TX}$ & RFA & RFA & $\mathrm{TX}$ & RFA & DN & 63.27 & 58486.24 \\
\hline 93 & RFA & $\mathrm{TX}$ & RFA & RFA & RFA & $\mathrm{TX}$ & Sorafenib & 62.74 & 50322.97 \\
\hline 94 & RFA & $\mathrm{TX}$ & RFA & RFA & RFA & $\mathrm{TX}$ & DN & 62.71 & 50282.44 \\
\hline 95 & RFA & $\mathrm{TX}$ & RFA & RFA & RFA & RFA & Sorafenib & 62.59 & 48142.21 \\
\hline 96 & RFA & $\mathrm{TX}$ & RFA & RFA & RFA & RFA & DN & 62.56 & 48101.68 \\
\hline 97 & RFA & RFA & $\mathrm{TX}$ & $\mathrm{TX}$ & $\mathrm{TX}$ & $\mathrm{TX}$ & Sorafenib & 75.54 & 237920.05 \\
\hline 98 & RFA & RFA & $\mathrm{TX}$ & $\mathrm{TX}$ & $\mathrm{TX}$ & $\mathrm{TX}$ & $\mathrm{DN}$ & 75.52 & 237879.51 \\
\hline 99 & RFA & RFA & TX & $\mathrm{TX}$ & $\mathrm{TX}$ & RFA & Sorafenib & 75.39 & 235739.29 \\
\hline 100 & RFA & RFA & $\mathrm{TX}$ & $\mathrm{TX}$ & $\mathrm{TX}$ & RFA & DN & 75.37 & 235698.75 \\
\hline 101 & RFA & RFA & TX & $\mathrm{TX}$ & RFA & $\mathrm{TX}$ & Sorafenib & 74.83 & 227535.49 \\
\hline 102 & RFA & RFA & $\mathrm{TX}$ & $\mathrm{TX}$ & RFA & $\mathrm{TX}$ & $\mathrm{DN}$ & 74.81 & 227494.95 \\
\hline 103 & RFA & RFA & $\mathrm{TX}$ & $\mathrm{TX}$ & RFA & RFA & Sorafenib & 74.68 & 225354.73 \\
\hline 104 & RFA & RFA & TX & $\mathrm{TX}$ & RFA & RFA & DN & 74.66 & 225314.19 \\
\hline 105 & RFA & RFA & $\mathrm{TX}$ & RFA & $\mathrm{TX}$ & $\mathrm{TX}$ & Sorafenib & 72.26 & 189787.61 \\
\hline 106 & RFA & RFA & $\mathrm{TX}$ & RFA & $\mathrm{TX}$ & $\mathrm{TX}$ & $\mathrm{DN}$ & 72.23 & 189747.08 \\
\hline 107 & RFA & RFA & $\mathrm{TX}$ & RFA & $\mathrm{TX}$ & RFA & Sorafenib & 72.11 & 187606.85 \\
\hline 108 & RFA & RFA & $\mathrm{TX}$ & RFA & $\mathrm{TX}$ & RFA & $\mathrm{DN}$ & 72.08 & 187566.32 \\
\hline 109 & RFA & RFA & $\mathrm{TX}$ & RFA & RFA & $\mathrm{TX}$ & Sorafenib & 71.55 & 179403.05 \\
\hline 110 & RFA & RFA & $\mathrm{TX}$ & RFA & RFA & $\mathrm{TX}$ & $\mathrm{DN}$ & 71.52 & 179362.52 \\
\hline 111 & RFA & RFA & $\mathrm{TX}$ & RFA & RFA & RFA & Sorafenib & 71.40 & 177222.29 \\
\hline 112 & RFA & RFA & TX & RFA & RFA & RFA & $\mathrm{DN}$ & 71.37 & 177181.76 \\
\hline
\end{tabular}




\begin{tabular}{|c|c|c|c|c|c|c|c|c|c|c|}
\hline 113 & RFA & RFA & RFA & $\mathrm{TX}$ & $\mathrm{TX}$ & $\mathrm{TX}$ & Sorafenib & 64.79 & 80412.23 & \\
\hline 114 & RFA & RFA & RFA & $\mathrm{TX}$ & $\mathrm{TX}$ & $\mathrm{TX}$ & $\mathrm{DN}$ & 64.76 & 80371.70 & \\
\hline 115 & RFA & RFA & RFA & $\mathrm{TX}$ & $\mathrm{TX}$ & RFA & Sorafenib & 64.64 & 78231.48 & \\
\hline 116 & RFA & RFA & RFA & $\mathrm{TX}$ & TX & RFA & DN & 64.62 & 78190.94 & \\
\hline 117 & RFA & RFA & RFA & $\mathrm{TX}$ & RFA & $\mathrm{TX}$ & Sorafenib & 64.08 & 70027.67 & \\
\hline 118 & RFA & RFA & RFA & $\mathrm{TX}$ & RFA & $\mathrm{TX}$ & $\mathrm{DN}$ & 64.06 & 69987.14 & \\
\hline 119 & RFA & RFA & RFA & $\mathrm{TX}$ & RFA & RFA & Sorafenib & 63.93 & 67846.92 & \\
\hline 120 & RFA & RFA & RFA & TX & RFA & RFA & $\mathrm{DN}$ & 63.91 & 67806.38 & \\
\hline 121 & RFA & RFA & RFA & RFA & $\mathrm{TX}$ & $\mathrm{TX}$ & Sorafenib & 61.50 & 32279.80 & \\
\hline 122 & RFA & RFA & RFA & RFA & $\mathrm{TX}$ & $\mathrm{TX}$ & $\mathrm{DN}$ & 61.48 & 32239.26 & \\
\hline 123 & RFA & RFA & RFA & RFA & $\mathrm{TX}$ & RFA & Sorafenib & 61.36 & 30099.04 & \\
\hline 124 & RFA & RFA & RFA & RFA & $\mathrm{TX}$ & RFA & DN & 61.33 & 30058.51 & \\
\hline 125 & RFA & RFA & RFA & RFA & RFA & $\mathrm{TX}$ & Sorafenib & 60.80 & 21895.24 & \\
\hline 126 & RFA & RFA & RFA & RFA & RFA & $\mathrm{TX}$ & DN & 60.77 & 21854.70 & \\
\hline 127 & RFA & RFA & RFA & RFA & RFA & RFA & Sorafenib & 60.65 & 19714.48 & \\
\hline 128 & RFA & RFA & RFA & RFA & RFA & RFA & $\mathrm{DN}$ & 60.62 & 19673.95 & \\
\hline 129 & DN & $\mathrm{TX}$ & $\mathrm{TX}$ & $\mathrm{TX}$ & $\mathrm{TX}$ & $\mathrm{TX}$ & Sorafenib & 76.25 & 257760.98 & $\mathrm{X}$ \\
\hline 130 & DN & $\mathrm{TX}$ & $\mathrm{TX}$ & $\mathrm{TX}$ & $\mathrm{TX}$ & $\mathrm{TX}$ & DN & 76.22 & 257727.44 & $\mathrm{X}$ \\
\hline 131 & $\mathrm{DN}$ & $\mathrm{TX}$ & $\mathrm{TX}$ & $\mathrm{TX}$ & $\mathrm{TX}$ & $\mathrm{DN}$ & Sorafenib & 75.01 & 257698.05 & $\mathrm{X}$ \\
\hline 132 & DN & $\mathrm{TX}$ & $\mathrm{TX}$ & $\mathrm{TX}$ & $\mathrm{TX}$ & $\mathrm{DN}$ & $\mathrm{DN}$ & 74.99 & 257664.52 & $\mathrm{X}$ \\
\hline 133 & DN & $\mathrm{TX}$ & $\mathrm{TX}$ & $\mathrm{TX}$ & $\mathrm{DN}$ & $\mathrm{TX}$ & Sorafenib & 73.00 & 246647.66 & $\mathrm{X}$ \\
\hline 134 & $\mathrm{DN}$ & $\mathrm{TX}$ & $\mathrm{TX}$ & $\mathrm{TX}$ & DN & $\mathrm{TX}$ & $\mathrm{DN}$ & 72.97 & 246614.13 & $\mathrm{X}$ \\
\hline 135 & DN & $\mathrm{TX}$ & $\mathrm{TX}$ & TX & $\mathrm{DN}$ & $\mathrm{DN}$ & Sorafenib & 71.77 & 246584.74 & $\mathrm{X}$ \\
\hline 136 & DN & $\mathrm{TX}$ & $\mathrm{TX}$ & $\mathrm{TX}$ & $\mathrm{DN}$ & $\mathrm{DN}$ & DN & 71.74 & 246551.20 & $\mathrm{X}$ \\
\hline 137 & DN & $\mathrm{TX}$ & $\mathrm{TX}$ & DN & $\mathrm{TX}$ & $\mathrm{TX}$ & Sorafenib & 59.98 & 202111.27 & $\mathrm{X}$ \\
\hline 138 & $\mathrm{DN}$ & $\mathrm{TX}$ & $\mathrm{TX}$ & DN & $\mathrm{TX}$ & $\mathrm{TX}$ & $\mathrm{DN}$ & 59.96 & 202077.73 & $\mathrm{X}$ \\
\hline 139 & DN & $\mathrm{TX}$ & $\mathrm{TX}$ & DN & $\mathrm{TX}$ & DN & Sorafenib & 58.75 & 202048.34 & $\mathrm{X}$ \\
\hline 140 & $\mathrm{DN}$ & $\mathrm{TX}$ & $\mathrm{TX}$ & DN & $\mathrm{TX}$ & $\mathrm{DN}$ & DN & 58.73 & 202014.80 & $\mathrm{X}$ \\
\hline 141 & $\mathrm{DN}$ & $\mathrm{TX}$ & $\mathrm{TX}$ & DN & $\mathrm{DN}$ & $\mathrm{TX}$ & Sorafenib & 56.73 & 190997.95 & $\mathrm{X}$ \\
\hline 142 & $\mathrm{DN}$ & $\mathrm{TX}$ & $\mathrm{TX}$ & DN & $\mathrm{DN}$ & $\mathrm{TX}$ & DN & 56.71 & 190964.42 & $\mathrm{X}$ \\
\hline 143 & $\mathrm{DN}$ & $\mathrm{TX}$ & $\mathrm{TX}$ & DN & DN & $\mathrm{DN}$ & Sorafenib & 55.50 & 190935.02 & $\mathrm{X}$ \\
\hline 144 & $\mathrm{DN}$ & $\mathrm{TX}$ & $\mathrm{TX}$ & DN & $\mathrm{DN}$ & $\mathrm{DN}$ & DN & 55.48 & 190901.49 & $\mathrm{X}$ \\
\hline 145 & $\mathrm{DN}$ & $\mathrm{TX}$ & $\mathrm{DN}$ & $\mathrm{TX}$ & $\mathrm{TX}$ & $\mathrm{TX}$ & Sorafenib & 28.96 & 95966.65 & $\mathrm{X}$ \\
\hline 146 & DN & $\mathrm{TX}$ & $\mathrm{DN}$ & $\mathrm{TX}$ & $\mathrm{TX}$ & $\mathrm{TX}$ & $\mathrm{DN}$ & 28.93 & 95933.11 & $\mathrm{X}$ \\
\hline 147 & $\mathrm{DN}$ & $\mathrm{TX}$ & $\mathrm{DN}$ & TX & $\mathrm{TX}$ & DN & Sorafenib & 27.73 & 95903.72 & $\mathrm{X}$ \\
\hline 148 & $\mathrm{DN}$ & $\mathrm{TX}$ & $\mathrm{DN}$ & $\mathrm{TX}$ & $\mathrm{TX}$ & $\mathrm{DN}$ & $\mathrm{DN}$ & 27.70 & 95870.18 & $\mathrm{X}$ \\
\hline 149 & DN & $\mathrm{TX}$ & $\mathrm{DN}$ & TX & DN & $\mathrm{TX}$ & Sorafenib & 25.71 & 84853.33 & X \\
\hline 150 & $\mathrm{DN}$ & $\mathrm{TX}$ & $\mathrm{DN}$ & $\mathrm{TX}$ & DN & $\mathrm{TX}$ & $\mathrm{DN}$ & 25.69 & 84819.80 & $\mathrm{X}$ \\
\hline 151 & $\mathrm{DN}$ & $\mathrm{TX}$ & $\mathrm{DN}$ & $\mathrm{TX}$ & $\mathrm{DN}$ & $\mathrm{DN}$ & Sorafenib & 24.48 & 84790.40 & $\mathrm{X}$ \\
\hline 152 & $\mathrm{DN}$ & $\mathrm{TX}$ & $\mathrm{DN}$ & $\mathrm{TX}$ & DN & DN & DN & 24.45 & 84756.87 & $\mathrm{X}$ \\
\hline 153 & $\mathrm{DN}$ & $\mathrm{TX}$ & $\mathrm{DN}$ & DN & $\mathrm{TX}$ & $\mathrm{TX}$ & Sorafenib & 12.69 & 40316.93 & $\mathrm{X}$ \\
\hline 154 & $\mathrm{DN}$ & $\mathrm{TX}$ & $\mathrm{DN}$ & DN & $\mathrm{TX}$ & $\mathrm{TX}$ & $\mathrm{DN}$ & 12.67 & 40283.40 & $\mathrm{X}$ \\
\hline 155 & $\mathrm{DN}$ & $\mathrm{TX}$ & $\mathrm{DN}$ & $\mathrm{DN}$ & $\mathrm{TX}$ & $\mathrm{DN}$ & Sorafenib & 11.46 & 40254.01 & $\mathrm{X}$ \\
\hline 156 & $\mathrm{DN}$ & $\mathrm{TX}$ & $\mathrm{DN}$ & DN & $\mathrm{TX}$ & $\mathrm{DN}$ & $\mathrm{DN}$ & 11.44 & 40220.47 & $\mathrm{X}$ \\
\hline 157 & $\mathrm{DN}$ & $\mathrm{TX}$ & $\mathrm{DN}$ & DN & DN & $\mathrm{TX}$ & Sorafenib & 9.44 & 29203.62 & $X$ \\
\hline 158 & $\mathrm{DN}$ & $\mathrm{TX}$ & $\mathrm{DN}$ & $\mathrm{DN}$ & $\mathrm{DN}$ & $\mathrm{TX}$ & $\mathrm{DN}$ & 9.42 & 29170.09 & $\mathrm{X}$ \\
\hline
\end{tabular}




\begin{tabular}{|c|c|c|c|c|c|c|c|c|c|c|}
\hline 159 & DN & $\mathrm{TX}$ & $\mathrm{DN}$ & $\mathrm{DN}$ & $\mathrm{DN}$ & DN & Sorafenib & 8.21 & 29140.69 & $X$ \\
\hline 160 & DN & $\mathrm{TX}$ & $\mathrm{DN}$ & $\mathrm{DN}$ & $\mathrm{DN}$ & DN & DN & 8.19 & 29107.16 & $\mathrm{X}$ \\
\hline 161 & DN & $\mathrm{DN}$ & $\mathrm{TX}$ & $\mathrm{TX}$ & $\mathrm{TX}$ & $\mathrm{TX}$ & Sorafenib & 68.06 & 229742.12 & $X$ \\
\hline 162 & DN & $\mathrm{DN}$ & $\mathrm{TX}$ & $\mathrm{TX}$ & $\mathrm{TX}$ & $\mathrm{TX}$ & DN & 68.03 & 229708.59 & $\mathrm{X}$ \\
\hline 163 & DN & $\mathrm{DN}$ & $\mathrm{TX}$ & $\mathrm{TX}$ & $\mathrm{TX}$ & DN & Sorafenib & 66.83 & 229679.20 & $\mathrm{X}$ \\
\hline 164 & DN & $\mathrm{DN}$ & $\mathrm{TX}$ & $\mathrm{TX}$ & $\mathrm{TX}$ & DN & $\mathrm{DN}$ & 66.80 & 229645.66 & $\mathrm{X}$ \\
\hline 165 & DN & $\mathrm{DN}$ & $\mathrm{TX}$ & $\mathrm{TX}$ & $\mathrm{DN}$ & $\mathrm{TX}$ & Sorafenib & 64.81 & 218628.81 & $\mathrm{X}$ \\
\hline 166 & DN & $\mathrm{DN}$ & $\mathrm{TX}$ & $\mathrm{TX}$ & $\mathrm{DN}$ & $\mathrm{TX}$ & DN & 64.78 & 218595.28 & $\mathrm{X}$ \\
\hline 167 & DN & $\mathrm{DN}$ & $\mathrm{TX}$ & $\mathrm{TX}$ & $\mathrm{DN}$ & DN & Sorafenib & 63.58 & 218565.88 & $\mathrm{X}$ \\
\hline 168 & DN & $\mathrm{DN}$ & $\mathrm{TX}$ & $\mathrm{TX}$ & $\mathrm{DN}$ & DN & DN & 63.55 & 218532.35 & $\mathrm{X}$ \\
\hline 169 & DN & $\mathrm{DN}$ & $\mathrm{TX}$ & $\mathrm{DN}$ & $\mathrm{TX}$ & $\mathrm{TX}$ & Sorafenib & 51.79 & 174092.41 & $X$ \\
\hline 170 & DN & $\mathrm{DN}$ & $\mathrm{TX}$ & $\mathrm{DN}$ & $\mathrm{TX}$ & $\mathrm{TX}$ & DN & 51.77 & 174058.88 & $\mathrm{X}$ \\
\hline 171 & DN & $\mathrm{DN}$ & $\mathrm{TX}$ & $\mathrm{DN}$ & $\mathrm{TX}$ & DN & Sorafenib & 50.56 & 174029.48 & $X$ \\
\hline 172 & DN & $\mathrm{DN}$ & $\mathrm{TX}$ & $\mathrm{DN}$ & $\mathrm{TX}$ & DN & DN & 50.54 & 173995.95 & $\mathrm{X}$ \\
\hline 173 & DN & $\mathrm{DN}$ & $\mathrm{TX}$ & $\mathrm{DN}$ & $\mathrm{DN}$ & $\mathrm{TX}$ & Sorafenib & 48.54 & 162979.10 & $\mathrm{X}$ \\
\hline 174 & DN & $\mathrm{DN}$ & $\mathrm{TX}$ & $\mathrm{DN}$ & $\mathrm{DN}$ & $\mathrm{TX}$ & DN & 48.52 & 162945.56 & $\mathrm{X}$ \\
\hline 175 & DN & $\mathrm{DN}$ & $\mathrm{TX}$ & $\mathrm{DN}$ & $\mathrm{DN}$ & DN & Sorafenib & 47.31 & 162916.17 & $\mathrm{X}$ \\
\hline 176 & DN & $\mathrm{DN}$ & $\mathrm{TX}$ & $\mathrm{DN}$ & $\mathrm{DN}$ & DN & DN & 47.29 & 162882.64 & $\mathrm{X}$ \\
\hline 177 & DN & $\mathrm{DN}$ & $\mathrm{DN}$ & $\mathrm{TX}$ & $\mathrm{TX}$ & $\mathrm{TX}$ & Sorafenib & 20.77 & 67947.79 & $X$ \\
\hline 178 & DN & $\mathrm{DN}$ & $\mathrm{DN}$ & $\mathrm{TX}$ & $\mathrm{TX}$ & $\mathrm{TX}$ & DN & 20.74 & 67914.26 & $\mathrm{X}$ \\
\hline 179 & DN & $\mathrm{DN}$ & $\mathrm{DN}$ & $\mathrm{TX}$ & $\mathrm{TX}$ & DN & Sorafenib & 19.54 & 67884.86 & $X$ \\
\hline 180 & DN & $\mathrm{DN}$ & $\mathrm{DN}$ & $\mathrm{TX}$ & $\mathrm{TX}$ & DN & DN & 19.51 & 67851.33 & $\mathrm{X}$ \\
\hline 181 & DN & $\mathrm{DN}$ & $\mathrm{DN}$ & $\mathrm{TX}$ & $\mathrm{DN}$ & $\mathrm{TX}$ & Sorafenib & 17.52 & 56834.48 & $X$ \\
\hline 182 & DN & $\mathrm{DN}$ & $\mathrm{DN}$ & $\mathrm{TX}$ & $\mathrm{DN}$ & TX & DN & 17.50 & 56800.94 & $X$ \\
\hline 183 & DN & $\mathrm{DN}$ & $\mathrm{DN}$ & $\mathrm{TX}$ & $\mathrm{DN}$ & DN & Sorafenib & 16.29 & 56771.55 & $\mathrm{X}$ \\
\hline 184 & DN & $\mathrm{DN}$ & $\mathrm{DN}$ & $\mathrm{TX}$ & $\mathrm{DN}$ & DN & DN & 16.26 & 56738.02 & $\mathrm{X}$ \\
\hline 185 & DN & $\mathrm{DN}$ & $\mathrm{DN}$ & $\mathrm{DN}$ & $\mathrm{TX}$ & $\mathrm{TX}$ & Sorafenib & 4.50 & 12298.08 & $\mathrm{X}$ \\
\hline 186 & DN & $\mathrm{DN}$ & $\mathrm{DN}$ & $\mathrm{DN}$ & $\mathrm{TX}$ & $\mathrm{TX}$ & DN & 4.48 & 12264.55 & $\mathrm{X}$ \\
\hline 187 & DN & $\mathrm{DN}$ & $\mathrm{DN}$ & $\mathrm{DN}$ & $\mathrm{TX}$ & DN & Sorafenib & 3.27 & 12235.15 & $\mathrm{X}$ \\
\hline 188 & DN & $\mathrm{DN}$ & $\mathrm{DN}$ & $\mathrm{DN}$ & $\mathrm{TX}$ & DN & DN & 3.25 & 12201.62 & $\mathrm{X}$ \\
\hline 189 & DN & $\mathrm{DN}$ & $\mathrm{DN}$ & $\mathrm{DN}$ & $\mathrm{DN}$ & $\mathrm{TX}$ & Sorafenib & 1.26 & 1184.77 & \\
\hline 190 & DN & $\mathrm{DN}$ & $\mathrm{DN}$ & $\mathrm{DN}$ & $\mathrm{DN}$ & $\mathrm{TX}$ & DN & 1.23 & 1151.23 & \\
\hline 191 & DN & $\mathrm{DN}$ & $\mathrm{DN}$ & $\mathrm{DN}$ & $\mathrm{DN}$ & DN & Sorafenib & 0.02 & 1121.84 & \\
\hline 192 & DN & $\mathrm{DN}$ & $\mathrm{DN}$ & $\mathrm{DN}$ & $\mathrm{DN}$ & $\mathrm{DN}$ & $\mathrm{DN}$ & 0.00 & 1088.30 & \\
\hline
\end{tabular}

\section{Initial Calculated MELD [15, 20)}

\begin{tabular}{|c|c|c|c|c|c|c|c|c|c|c|}
\hline \multicolumn{6}{|c|}{ Month 1 Decision } & \multicolumn{5}{|c|}{ Damage } \\
\hline & $\begin{array}{c}\text { Initial } \\
\text { Decision }\end{array}$ & M1 & M2 & M3 & M4 & M5 & Drop & Lives & Cost & $\mathrm{P}-\mathrm{O} ?$ \\
\hline 1 & TACE & $\mathrm{TX}$ & $\mathrm{TX}$ & $\mathrm{TX}$ & $\mathrm{TX}$ & $\mathrm{TX}$ & Sorafenib & 77.72 & 271121.30 & \\
\hline 2 & TACE & $\mathrm{TX}$ & $\mathrm{TX}$ & $\mathrm{TX}$ & TX & TX & DN & 77.68 & 271062.86 & \\
\hline
\end{tabular}




\begin{tabular}{|c|c|c|c|c|c|c|c|c|c|}
\hline 3 & TACE & $\mathrm{TX}$ & $\mathrm{TX}$ & $\mathrm{TX}$ & $\mathrm{TX}$ & TACE & Sorafenib & 76.87 & 263916.34 \\
\hline 4 & TACE & $\mathrm{TX}$ & $\mathrm{TX}$ & $\mathrm{TX}$ & $\mathrm{TX}$ & TACE & $\mathrm{DN}$ & 76.83 & 263857.89 \\
\hline 5 & TACE & $\mathrm{TX}$ & $\mathrm{TX}$ & $\mathrm{TX}$ & TACE & $\mathrm{TX}$ & Sorafenib & 73.07 & 231493.99 \\
\hline 6 & TACE & $\mathrm{TX}$ & $\mathrm{TX}$ & $\mathrm{TX}$ & TACE & $\mathrm{TX}$ & $\mathrm{DN}$ & 73.03 & 231435.54 \\
\hline 7 & TACE & $\mathrm{TX}$ & $\mathrm{TX}$ & $\mathrm{TX}$ & TACE & TACE & Sorafenib & 72.22 & 224289.02 \\
\hline 8 & TACE & $\mathrm{TX}$ & $\mathrm{TX}$ & $\mathrm{TX}$ & TACE & TACE & $\mathrm{DN}$ & 72.18 & 224230.58 \\
\hline 9 & TACE & $\mathrm{TX}$ & $\mathrm{TX}$ & TACE & $\mathrm{TX}$ & $\mathrm{TX}$ & Sorafenib & 61.65 & 134201.57 \\
\hline 10 & TACE & $\mathrm{TX}$ & $\mathrm{TX}$ & TACE & $\mathrm{TX}$ & $\mathrm{TX}$ & $\mathrm{DN}$ & 61.61 & 134143.13 \\
\hline 11 & TACE & $\mathrm{TX}$ & $\mathrm{TX}$ & TACE & $\mathrm{TX}$ & TACE & Sorafenib & 60.80 & 126996.61 \\
\hline 12 & TACE & $\mathrm{TX}$ & $\mathrm{TX}$ & TACE & $\mathrm{TX}$ & TACE & $\mathrm{DN}$ & 60.77 & 126938.16 \\
\hline 13 & TACE & $\mathrm{TX}$ & $\mathrm{TX}$ & TACE & TACE & $\mathrm{TX}$ & Sorafenib & 57.00 & 94574.26 \\
\hline 14 & TACE & $\mathrm{TX}$ & $\mathrm{TX}$ & TACE & TACE & $\mathrm{TX}$ & $\mathrm{DN}$ & 56.96 & 94515.81 \\
\hline 15 & TACE & $\mathrm{TX}$ & $\mathrm{TX}$ & TACE & TACE & TACE & Sorafenib & 56.15 & 87369.29 \\
\hline 16 & TACE & $\mathrm{TX}$ & $\mathrm{TX}$ & TACE & TACE & TACE & $\mathrm{DN}$ & 56.12 & 87310.85 \\
\hline 17 & TACE & $\mathrm{TX}$ & TACE & $\mathrm{TX}$ & $\mathrm{TX}$ & $\mathrm{TX}$ & Sorafenib & 71.30 & 216475.19 \\
\hline 18 & TACE & $\mathrm{TX}$ & TACE & $\mathrm{TX}$ & $\mathrm{TX}$ & $\mathrm{TX}$ & $\mathrm{DN}$ & 71.27 & 216416.74 \\
\hline 19 & TACE & $\mathrm{TX}$ & TACE & $\mathrm{TX}$ & $\mathrm{TX}$ & TACE & Sorafenib & 70.46 & 209270.22 \\
\hline 20 & TACE & $\mathrm{TX}$ & TACE & $\mathrm{TX}$ & $\mathrm{TX}$ & TACE & $\mathrm{DN}$ & 70.42 & 209211.77 \\
\hline 21 & TACE & $\mathrm{TX}$ & TACE & $\mathrm{TX}$ & TACE & $\mathrm{TX}$ & Sorafenib & 66.65 & 176847.87 \\
\hline 22 & TACE & $\mathrm{TX}$ & TACE & $\mathrm{TX}$ & TACE & $\mathrm{TX}$ & $\mathrm{DN}$ & 66.62 & 176789.43 \\
\hline 23 & TACE & $\mathrm{TX}$ & TACE & $\mathrm{TX}$ & TACE & TACE & Sorafenib & 65.81 & 169642.90 \\
\hline 24 & TACE & $\mathrm{TX}$ & TACE & $\mathrm{TX}$ & TACE & TACE & $\mathrm{DN}$ & 65.77 & 169584.46 \\
\hline 25 & TACE & $\mathrm{TX}$ & TACE & TACE & $\mathrm{TX}$ & $\mathrm{TX}$ & Sorafenib & 55.24 & 79555.45 \\
\hline 26 & TACE & $\mathrm{TX}$ & TACE & TACE & $\mathrm{TX}$ & $\mathrm{TX}$ & $\mathrm{DN}$ & 55.20 & 79497.01 \\
\hline 27 & TACE & $\mathrm{TX}$ & TACE & TACE & $\mathrm{TX}$ & TACE & Sorafenib & 54.39 & 72350.49 \\
\hline 28 & TACE & $\mathrm{TX}$ & TACE & TACE & $\mathrm{TX}$ & TACE & DN & 54.35 & 72292.04 \\
\hline 29 & TACE & $\mathrm{TX}$ & TACE & TACE & TACE & $\mathrm{TX}$ & Sorafenib & 50.59 & 39928.14 \\
\hline 30 & TACE & $\mathrm{TX}$ & TACE & TACE & TACE & $\mathrm{TX}$ & $\mathrm{DN}$ & 50.55 & 39869.69 \\
\hline 31 & TACE & $\mathrm{TX}$ & TACE & TACE & TACE & TACE & Sorafenib & 49.74 & 32723.17 \\
\hline 32 & TACE & $\mathrm{TX}$ & TACE & TACE & TACE & TACE & $\mathrm{DN}$ & 49.70 & 32664.73 \\
\hline 33 & TACE & TACE & $\mathrm{TX}$ & $\mathrm{TX}$ & $\mathrm{TX}$ & $\mathrm{TX}$ & Sorafenib & 77.29 & 267518.82 \\
\hline 34 & TACE & TACE & $\mathrm{TX}$ & $\mathrm{TX}$ & $\mathrm{TX}$ & $\mathrm{TX}$ & DN & 77.26 & 267460.38 \\
\hline 35 & TACE & TACE & $\mathrm{TX}$ & $\mathrm{TX}$ & $\mathrm{TX}$ & TACE & Sorafenib & 76.45 & 260313.85 \\
\hline 36 & TACE & TACE & $\mathrm{TX}$ & $\mathrm{TX}$ & $\mathrm{TX}$ & TACE & $\mathrm{DN}$ & 76.41 & 260255.41 \\
\hline 37 & TACE & TACE & $\mathrm{TX}$ & $\mathrm{TX}$ & TACE & $\mathrm{TX}$ & Sorafenib & 72.64 & 227891.51 \\
\hline 38 & TACE & TACE & $\mathrm{TX}$ & $\mathrm{TX}$ & TACE & $\mathrm{TX}$ & $\mathrm{DN}$ & 72.61 & 227833.06 \\
\hline 39 & TACE & TACE & $\mathrm{TX}$ & $\mathrm{TX}$ & TACE & TACE & Sorafenib & 71.80 & 220686.54 \\
\hline 40 & TACE & TACE & $\mathrm{TX}$ & $\mathrm{TX}$ & TACE & TACE & $\mathrm{DN}$ & 71.76 & 220628.09 \\
\hline 41 & TACE & TACE & $\mathrm{TX}$ & TACE & $\mathrm{TX}$ & $\mathrm{TX}$ & Sorafenib & 61.23 & 130599.09 \\
\hline 42 & TACE & TACE & $\mathrm{TX}$ & TACE & $\mathrm{TX}$ & $\mathrm{TX}$ & $\mathrm{DN}$ & 61.19 & 130540.64 \\
\hline 43 & TACE & TACE & $\mathrm{TX}$ & TACE & $\mathrm{TX}$ & TACE & Sorafenib & 60.38 & 123394.12 \\
\hline 44 & TACE & TACE & $\mathrm{TX}$ & TACE & $\mathrm{TX}$ & TACE & DN & 60.34 & 123335.68 \\
\hline 45 & TACE & TACE & $\mathrm{TX}$ & TACE & TACE & $\mathrm{TX}$ & Sorafenib & 56.58 & 90971.77 \\
\hline 46 & TACE & TACE & $\mathrm{TX}$ & TACE & TACE & $\mathrm{TX}$ & DN & 56.54 & 90913.33 \\
\hline 47 & TACE & TACE & $\mathrm{TX}$ & TACE & TACE & TACE & Sorafenib & 55.73 & 83766.81 \\
\hline 48 & TACE & TACE & $\mathrm{TX}$ & TACE & TACE & TACE & $\mathrm{DN}$ & 55.69 & 83708.36 \\
\hline
\end{tabular}




\begin{tabular}{|c|c|c|c|c|c|c|c|c|c|}
\hline 49 & TACE & TACE & TACE & TX & $\mathrm{TX}$ & $\mathrm{TX}$ & Sorafenib & 70.88 & 212872.70 \\
\hline 50 & TACE & TACE & TACE & $\mathrm{TX}$ & TX & TX & $\mathrm{DN}$ & 70.84 & 212814.26 \\
\hline 51 & TACE & TACE & TACE & $\mathrm{TX}$ & TX & TACE & Sorafenib & 70.03 & 205667.74 \\
\hline 52 & TACE & TACE & TACE & $\mathrm{TX}$ & $\mathrm{TX}$ & TACE & $\mathrm{DN}$ & 70.00 & 205609.29 \\
\hline 53 & TACE & TACE & TACE & $\mathrm{TX}$ & TACE & $\mathrm{TX}$ & Sorafenib & 66.23 & 173245.39 \\
\hline 54 & TACE & TACE & TACE & $\mathrm{TX}$ & TACE & $\mathrm{TX}$ & $\mathrm{DN}$ & 66.19 & 173186.94 \\
\hline 55 & TACE & TACE & TACE & $\mathrm{TX}$ & TACE & TACE & Sorafenib & 65.38 & 166040.42 \\
\hline 56 & TACE & TACE & TACE & $\mathrm{TX}$ & TACE & TACE & $\mathrm{DN}$ & 65.35 & 165981.98 \\
\hline 57 & TACE & TACE & TACE & TACE & $\mathrm{TX}$ & TX & Sorafenib & 54.81 & 75952.97 \\
\hline 58 & TACE & TACE & TACE & TACE & $\mathrm{TX}$ & $\mathrm{TX}$ & DN & 54.78 & 75894.53 \\
\hline 59 & TACE & TACE & TACE & TACE & $\mathrm{TX}$ & TACE & Sorafenib & 53.97 & 68748.00 \\
\hline 60 & TACE & TACE & TACE & TACE & $\mathrm{TX}$ & TACE & DN & 53.93 & 68689.56 \\
\hline 61 & TACE & TACE & TACE & TACE & TACE & TX & Sorafenib & 50.16 & 36325.66 \\
\hline 62 & TACE & TACE & TACE & TACE & TACE & $\mathrm{TX}$ & DN & 50.13 & 36267.21 \\
\hline 63 & TACE & TACE & TACE & TACE & TACE & TACE & Sorafenib & 49.32 & 29120.69 \\
\hline 64 & TACE & TACE & TACE & TACE & TACE & TACE & $\mathrm{DN}$ & 49.28 & 29062.24 \\
\hline 65 & RFA & $\mathrm{TX}$ & $\mathrm{TX}$ & $\mathrm{TX}$ & $\mathrm{TX}$ & $\mathrm{TX}$ & Sorafenib & 77.48 & 266347.78 \\
\hline 66 & RFA & $\mathrm{TX}$ & $\mathrm{TX}$ & TX & $\mathrm{TX}$ & TX & $\mathrm{DN}$ & 77.46 & 266307.25 \\
\hline 67 & RFA & $\mathrm{TX}$ & $\mathrm{TX}$ & $\mathrm{TX}$ & $\mathrm{TX}$ & RFA & Sorafenib & 76.79 & 256274.76 \\
\hline 68 & RFA & $\mathrm{TX}$ & $\mathrm{TX}$ & $\mathrm{TX}$ & $\mathrm{TX}$ & RFA & DN & 76.77 & 256234.22 \\
\hline 69 & RFA & TX & $\mathrm{TX}$ & TX & RFA & TX & Sorafenib & 75.42 & 236154.67 \\
\hline 70 & RFA & $\mathrm{TX}$ & $\mathrm{TX}$ & $\mathrm{TX}$ & RFA & $\mathrm{TX}$ & $\mathrm{DN}$ & 75.39 & 236114.14 \\
\hline 71 & RFA & $\mathrm{TX}$ & $\mathrm{TX}$ & $\mathrm{TX}$ & RFA & RFA & Sorafenib & 74.73 & 226081.65 \\
\hline 72 & RFA & $\mathrm{TX}$ & $\mathrm{TX}$ & $\mathrm{TX}$ & RFA & RFA & $\mathrm{DN}$ & 74.71 & 226041.11 \\
\hline 73 & RFA & $\mathrm{TX}$ & $\mathrm{TX}$ & RFA & $\mathrm{TX}$ & $\mathrm{TX}$ & Sorafenib & 67.75 & 123741.81 \\
\hline 74 & RFA & $\mathrm{TX}$ & $\mathrm{TX}$ & RFA & $\mathrm{TX}$ & $\mathrm{TX}$ & $\mathrm{DN}$ & 67.72 & 123701.28 \\
\hline 75 & RFA & $\mathrm{TX}$ & $\mathrm{TX}$ & RFA & $\mathrm{TX}$ & RFA & Sorafenib & 67.06 & 113668.79 \\
\hline 76 & RFA & $\mathrm{TX}$ & $\mathrm{TX}$ & RFA & $\mathrm{TX}$ & RFA & $\mathrm{DN}$ & 67.03 & 113628.25 \\
\hline 77 & RFA & $\mathrm{TX}$ & $\mathrm{TX}$ & RFA & RFA & $\mathrm{TX}$ & Sorafenib & 65.69 & 93548.70 \\
\hline 78 & RFA & $\mathrm{TX}$ & TX & RFA & RFA & TX & DN & 65.66 & 93508.17 \\
\hline 79 & RFA & $\mathrm{TX}$ & $\mathrm{TX}$ & RFA & RFA & RFA & Sorafenib & 65.00 & 83475.68 \\
\hline 80 & RFA & $\mathrm{TX}$ & $\mathrm{TX}$ & RFA & RFA & RFA & DN & 64.97 & 83435.14 \\
\hline 81 & RFA & $\mathrm{TX}$ & RFA & $\mathrm{TX}$ & $\mathrm{TX}$ & $\mathrm{TX}$ & Sorafenib & 73.59 & 209310.58 \\
\hline 82 & RFA & $\mathrm{TX}$ & RFA & $\mathrm{TX}$ & $\mathrm{TX}$ & $\mathrm{TX}$ & DN & 73.56 & 209270.05 \\
\hline 83 & RFA & $\mathrm{TX}$ & RFA & $\mathrm{TX}$ & $\mathrm{TX}$ & RFA & Sorafenib & 72.90 & 199237.56 \\
\hline 84 & RFA & $\mathrm{TX}$ & RFA & $\mathrm{TX}$ & $\mathrm{TX}$ & RFA & $\mathrm{DN}$ & 72.87 & 199197.03 \\
\hline 85 & RFA & $\mathrm{TX}$ & RFA & $\mathrm{TX}$ & RFA & $\mathrm{TX}$ & Sorafenib & 71.53 & 179117.48 \\
\hline 86 & RFA & $\mathrm{TX}$ & RFA & $\mathrm{TX}$ & RFA & TX & $\mathrm{DN}$ & 71.50 & 179076.94 \\
\hline 87 & RFA & $\mathrm{TX}$ & RFA & $\mathrm{TX}$ & RFA & RFA & Sorafenib & 70.84 & 169044.45 \\
\hline 88 & RFA & $\mathrm{TX}$ & RFA & $\mathrm{TX}$ & RFA & RFA & DN & 70.81 & 169003.92 \\
\hline 89 & RFA & $\mathrm{TX}$ & RFA & RFA & $\mathrm{TX}$ & $\mathrm{TX}$ & Sorafenib & 63.85 & 66704.61 \\
\hline 90 & RFA & $\mathrm{TX}$ & RFA & RFA & $\mathrm{TX}$ & $\mathrm{TX}$ & DN & 63.83 & 66664.08 \\
\hline 91 & RFA & $\mathrm{TX}$ & RFA & RFA & $\mathrm{TX}$ & RFA & Sorafenib & 63.17 & 56631.59 \\
\hline 92 & RFA & $\mathrm{TX}$ & RFA & RFA & $\mathrm{TX}$ & RFA & DN & 63.14 & 56591.06 \\
\hline 93 & RFA & $\mathrm{TX}$ & RFA & RFA & RFA & TX & Sorafenib & 61.79 & 36511.51 \\
\hline 94 & RFA & $\mathrm{TX}$ & RFA & RFA & RFA & $\mathrm{TX}$ & $\mathrm{DN}$ & 61.77 & 36470.97 \\
\hline
\end{tabular}




\begin{tabular}{|c|c|c|c|c|c|c|c|c|c|}
\hline 95 & RFA & $\mathrm{TX}$ & RFA & RFA & RFA & RFA & Sorafenib & 61.11 & 26438.48 \\
\hline 96 & RFA & $\mathrm{TX}$ & RFA & RFA & RFA & RFA & DN & 61.08 & 26397.95 \\
\hline 97 & RFA & RFA & $\mathrm{TX}$ & $\mathrm{TX}$ & $\mathrm{TX}$ & $\mathrm{TX}$ & Sorafenib & 77.02 & 259623.78 \\
\hline 98 & RFA & RFA & $\mathrm{TX}$ & $\mathrm{TX}$ & $\mathrm{TX}$ & $\mathrm{TX}$ & DN & 77.00 & 259583.24 \\
\hline 99 & RFA & RFA & $\mathrm{TX}$ & $\mathrm{TX}$ & $\mathrm{TX}$ & RFA & Sorafenib & 76.33 & 249550.75 \\
\hline 100 & RFA & RFA & $\mathrm{TX}$ & TX & $\mathrm{TX}$ & RFA & DN & 76.31 & 249510.22 \\
\hline 101 & RFA & RFA & $\mathrm{TX}$ & $\mathrm{TX}$ & RFA & $\mathrm{TX}$ & Sorafenib & 74.96 & 229430.67 \\
\hline 102 & RFA & RFA & $\mathrm{TX}$ & TX & RFA & $\mathrm{TX}$ & $\mathrm{DN}$ & 74.94 & 229390.13 \\
\hline 103 & RFA & RFA & $\mathrm{TX}$ & $\mathrm{TX}$ & RFA & RFA & Sorafenib & 74.27 & 219357.65 \\
\hline 104 & RFA & RFA & $\mathrm{TX}$ & $\mathrm{TX}$ & RFA & RFA & DN & 74.25 & 219317.11 \\
\hline 105 & RFA & RFA & $\mathrm{TX}$ & RFA & $\mathrm{TX}$ & $\mathrm{TX}$ & Sorafenib & 67.29 & 117017.81 \\
\hline 106 & RFA & RFA & $\mathrm{TX}$ & RFA & $\mathrm{TX}$ & $\mathrm{TX}$ & DN & 67.26 & 116977.27 \\
\hline 107 & RFA & RFA & $\mathrm{TX}$ & RFA & $\mathrm{TX}$ & RFA & Sorafenib & 66.60 & 106944.78 \\
\hline 108 & RFA & RFA & $\mathrm{TX}$ & RFA & $\mathrm{TX}$ & RFA & DN & 66.58 & 106904.25 \\
\hline 109 & RFA & RFA & $\mathrm{TX}$ & RFA & RFA & $\mathrm{TX}$ & Sorafenib & 65.23 & 86824.70 \\
\hline 110 & RFA & RFA & $\mathrm{TX}$ & RFA & RFA & $\mathrm{TX}$ & DN & 65.20 & 86784.16 \\
\hline 111 & RFA & RFA & $\mathrm{TX}$ & RFA & RFA & RFA & Sorafenib & 64.54 & 76751.68 \\
\hline 112 & RFA & RFA & $\mathrm{TX}$ & RFA & RFA & RFA & $\mathrm{DN}$ & 64.51 & 76711.14 \\
\hline 113 & RFA & RFA & RFA & $\mathrm{TX}$ & $\mathrm{TX}$ & $\mathrm{TX}$ & Sorafenib & 73.13 & 202586.58 \\
\hline 114 & RFA & RFA & RFA & $\mathrm{TX}$ & $\mathrm{TX}$ & $\mathrm{TX}$ & DN & 73.10 & 202546.05 \\
\hline 115 & RFA & RFA & RFA & $\mathrm{TX}$ & $\mathrm{TX}$ & RFA & Sorafenib & 72.44 & 192513.56 \\
\hline 116 & RFA & RFA & RFA & TX & $\mathrm{TX}$ & RFA & DN & 72.42 & 192473.02 \\
\hline 117 & RFA & RFA & RFA & TX & RFA & $\mathrm{TX}$ & Sorafenib & 71.07 & 172393.47 \\
\hline 118 & RFA & RFA & RFA & $\mathrm{TX}$ & RFA & $\mathrm{TX}$ & $\mathrm{DN}$ & 71.04 & 172352.94 \\
\hline 119 & RFA & RFA & RFA & $\mathrm{TX}$ & RFA & RFA & Sorafenib & 70.38 & 162320.45 \\
\hline 120 & RFA & RFA & RFA & $\mathrm{TX}$ & RFA & RFA & DN & 70.36 & 162279.92 \\
\hline 121 & RFA & RFA & RFA & RFA & $\mathrm{TX}$ & $\mathrm{TX}$ & Sorafenib & 63.40 & 59980.61 \\
\hline 122 & RFA & RFA & RFA & RFA & $\mathrm{TX}$ & $\mathrm{TX}$ & DN & 63.37 & 59940.08 \\
\hline 123 & RFA & RFA & RFA & RFA & $\mathrm{TX}$ & RFA & Sorafenib & 62.71 & 49907.59 \\
\hline 124 & RFA & RFA & RFA & RFA & $\mathrm{TX}$ & RFA & DN & 62.68 & 49867.05 \\
\hline 125 & RFA & RFA & RFA & RFA & RFA & $\mathrm{TX}$ & Sorafenib & 61.33 & 29787.50 \\
\hline 126 & RFA & RFA & RFA & RFA & RFA & $\mathrm{TX}$ & DN & 61.31 & 29746.97 \\
\hline 127 & RFA & RFA & RFA & RFA & RFA & RFA & Sorafenib & 60.65 & 19714.48 \\
\hline 128 & RFA & RFA & RFA & RFA & RFA & RFA & DN & 60.62 & 19673.95 \\
\hline 129 & DN & $\mathrm{TX}$ & $\mathrm{TX}$ & $\mathrm{TX}$ & $\mathrm{TX}$ & $\mathrm{TX}$ & Sorafenib & 76.25 & 255112.88 \\
\hline 130 & DN & $\mathrm{TX}$ & $\mathrm{TX}$ & $\mathrm{TX}$ & $\mathrm{TX}$ & $\mathrm{TX}$ & $\mathrm{DN}$ & 76.22 & 255079.34 \\
\hline 131 & DN & $\mathrm{TX}$ & $\mathrm{TX}$ & $\mathrm{TX}$ & $\mathrm{TX}$ & $\mathrm{DN}$ & Sorafenib & 74.23 & 255009.79 \\
\hline 132 & DN & $\mathrm{TX}$ & $\mathrm{TX}$ & $\mathrm{TX}$ & $\mathrm{TX}$ & $\mathrm{DN}$ & DN & 74.20 & 254976.26 \\
\hline 133 & $\mathrm{DN}$ & $\mathrm{TX}$ & $\mathrm{TX}$ & $\mathrm{TX}$ & $\mathrm{DN}$ & $\mathrm{TX}$ & Sorafenib & 64.40 & 214595.01 \\
\hline 134 & DN & $\mathrm{TX}$ & $\mathrm{TX}$ & $\mathrm{TX}$ & $\mathrm{DN}$ & $\mathrm{TX}$ & DN & 64.38 & 214561.48 \\
\hline 135 & $\mathrm{DN}$ & $\mathrm{TX}$ & $\mathrm{TX}$ & TX & $\mathrm{DN}$ & $\mathrm{DN}$ & Sorafenib & 62.39 & 214491.92 \\
\hline 136 & $\mathrm{DN}$ & $\mathrm{TX}$ & $\mathrm{TX}$ & TX & $\mathrm{DN}$ & $\mathrm{DN}$ & $\mathrm{DN}$ & 62.36 & 214458.39 \\
\hline 137 & $\mathrm{DN}$ & $\mathrm{TX}$ & $\mathrm{TX}$ & $\mathrm{DN}$ & $\mathrm{TX}$ & $\mathrm{TX}$ & Sorafenib & 28.18 & 90658.00 \\
\hline 138 & $\mathrm{DN}$ & $\mathrm{TX}$ & $\mathrm{TX}$ & $\mathrm{DN}$ & $\mathrm{TX}$ & $\mathrm{TX}$ & DN & 28.16 & 90624.47 \\
\hline 139 & DN & $\mathrm{TX}$ & $\mathrm{TX}$ & $\mathrm{DN}$ & $\mathrm{TX}$ & $\mathrm{DN}$ & Sorafenib & 26.16 & 90554.92 \\
\hline 140 & DN & $\mathrm{TX}$ & $\mathrm{TX}$ & $\mathrm{DN}$ & $\mathrm{TX}$ & $\mathrm{DN}$ & $\mathrm{DN}$ & 26.14 & 90521.38 \\
\hline
\end{tabular}




\begin{tabular}{|c|c|c|c|c|c|c|c|c|c|}
\hline 141 & $\mathrm{DN}$ & $\mathrm{TX}$ & $\mathrm{TX}$ & DN & DN & $\mathrm{TX}$ & Sorafenib & 16.34 & 50140.13 \\
\hline 142 & $\mathrm{DN}$ & $\mathrm{TX}$ & $\mathrm{TX}$ & $\mathrm{DN}$ & $\mathrm{DN}$ & $\mathrm{TX}$ & $\mathrm{DN}$ & 16.31 & 50106.60 \\
\hline 143 & $\mathrm{DN}$ & $\mathrm{TX}$ & $\mathrm{TX}$ & $\mathrm{DN}$ & $\mathrm{DN}$ & $\mathrm{DN}$ & Sorafenib & 14.32 & 50037.05 \\
\hline 144 & $\mathrm{DN}$ & $\mathrm{TX}$ & $\mathrm{TX}$ & $\mathrm{DN}$ & $\mathrm{DN}$ & $\mathrm{DN}$ & $\mathrm{DN}$ & 14.30 & 50003.51 \\
\hline 145 & $\mathrm{DN}$ & $\mathrm{TX}$ & DN & $\mathrm{TX}$ & $\mathrm{TX}$ & $\mathrm{TX}$ & Sorafenib & 62.73 & 208885.93 \\
\hline 146 & $\mathrm{DN}$ & $\mathrm{TX}$ & $\mathrm{DN}$ & $\mathrm{TX}$ & $\mathrm{TX}$ & $\mathrm{TX}$ & $\mathrm{DN}$ & 62.71 & 208852.39 \\
\hline 147 & $\mathrm{DN}$ & $\mathrm{TX}$ & DN & $\mathrm{TX}$ & $\mathrm{TX}$ & $\mathrm{DN}$ & Sorafenib & 60.72 & 208782.84 \\
\hline 148 & $\mathrm{DN}$ & $\mathrm{TX}$ & DN & $\mathrm{TX}$ & $\mathrm{TX}$ & $\mathrm{DN}$ & $\mathrm{DN}$ & 60.69 & 208749.31 \\
\hline 149 & $\mathrm{DN}$ & $\mathrm{TX}$ & DN & $\mathrm{TX}$ & $\mathrm{DN}$ & $\mathrm{TX}$ & Sorafenib & 50.89 & 168368.06 \\
\hline 150 & $\mathrm{DN}$ & $\mathrm{TX}$ & DN & $\mathrm{TX}$ & $\mathrm{DN}$ & $\mathrm{TX}$ & $\mathrm{DN}$ & 50.87 & 168334.52 \\
\hline 151 & $\mathrm{DN}$ & $\mathrm{TX}$ & DN & $\mathrm{TX}$ & $\mathrm{DN}$ & $\mathrm{DN}$ & Sorafenib & 48.88 & 168264.97 \\
\hline 152 & $\mathrm{DN}$ & $\mathrm{TX}$ & DN & $\mathrm{TX}$ & $\mathrm{DN}$ & $\mathrm{DN}$ & $\mathrm{DN}$ & 48.85 & 168231.44 \\
\hline 153 & $\mathrm{DN}$ & $\mathrm{TX}$ & DN & $\mathrm{DN}$ & $\mathrm{TX}$ & $\mathrm{TX}$ & Sorafenib & 14.67 & 44431.05 \\
\hline 154 & $\mathrm{DN}$ & $\mathrm{TX}$ & DN & $\mathrm{DN}$ & $\mathrm{TX}$ & $\mathrm{TX}$ & $\mathrm{DN}$ & 14.64 & 44397.52 \\
\hline 155 & $\mathrm{DN}$ & $\mathrm{TX}$ & DN & $\mathrm{DN}$ & $\mathrm{TX}$ & $\mathrm{DN}$ & Sorafenib & 12.65 & 44327.96 \\
\hline 156 & $\mathrm{DN}$ & $\mathrm{TX}$ & DN & $\mathrm{DN}$ & $\mathrm{TX}$ & $\mathrm{DN}$ & $\mathrm{DN}$ & 12.63 & 44294.43 \\
\hline 157 & $\mathrm{DN}$ & $\mathrm{TX}$ & DN & $\mathrm{DN}$ & $\mathrm{DN}$ & $\mathrm{TX}$ & Sorafenib & 2.83 & 3913.18 \\
\hline 158 & $\mathrm{DN}$ & $\mathrm{TX}$ & DN & $\mathrm{DN}$ & $\mathrm{DN}$ & $\mathrm{TX}$ & $\mathrm{DN}$ & 2.80 & 3879.65 \\
\hline 159 & $\mathrm{DN}$ & $\mathrm{TX}$ & DN & $\mathrm{DN}$ & $\mathrm{DN}$ & $\mathrm{DN}$ & Sorafenib & 0.81 & 3810.10 \\
\hline 160 & $\mathrm{DN}$ & $\mathrm{TX}$ & $\mathrm{DN}$ & $\mathrm{DN}$ & $\mathrm{DN}$ & $\mathrm{DN}$ & $\mathrm{DN}$ & 0.79 & 3776.56 \\
\hline 161 & $\mathrm{DN}$ & $\mathrm{DN}$ & $\mathrm{TX}$ & $\mathrm{TX}$ & $\mathrm{TX}$ & $\mathrm{TX}$ & Sorafenib & 75.46 & 252424.62 \\
\hline 162 & $\mathrm{DN}$ & $\mathrm{DN}$ & $\mathrm{TX}$ & $\mathrm{TX}$ & $\mathrm{TX}$ & $\mathrm{TX}$ & $\mathrm{DN}$ & 75.44 & 252391.09 \\
\hline 163 & $\mathrm{DN}$ & $\mathrm{DN}$ & $\mathrm{TX}$ & $\mathrm{TX}$ & $\mathrm{TX}$ & $\mathrm{DN}$ & Sorafenib & 73.44 & 252321.53 \\
\hline 164 & $\mathrm{DN}$ & $\mathrm{DN}$ & $\mathrm{TX}$ & $\mathrm{TX}$ & $\mathrm{TX}$ & $\mathrm{DN}$ & $\mathrm{DN}$ & 73.42 & 252288.00 \\
\hline 165 & $\mathrm{DN}$ & $\mathrm{DN}$ & $\mathrm{TX}$ & $\mathrm{TX}$ & $\mathrm{DN}$ & $\mathrm{TX}$ & Sorafenib & 63.62 & 211906.75 \\
\hline 166 & $\mathrm{DN}$ & DN & $\mathrm{TX}$ & $\mathrm{TX}$ & $\mathrm{DN}$ & $\mathrm{TX}$ & DN & 63.59 & 211873.22 \\
\hline 167 & $\mathrm{DN}$ & $\mathrm{DN}$ & $\mathrm{TX}$ & $\mathrm{TX}$ & $\mathrm{DN}$ & $\mathrm{DN}$ & Sorafenib & 61.60 & 211803.67 \\
\hline 168 & $\mathrm{DN}$ & $\mathrm{DN}$ & $\mathrm{TX}$ & $\mathrm{TX}$ & $\mathrm{DN}$ & $\mathrm{DN}$ & $\mathrm{DN}$ & 61.58 & 211770.13 \\
\hline 169 & $\mathrm{DN}$ & $\mathrm{DN}$ & $\mathrm{TX}$ & $\mathrm{DN}$ & $\mathrm{TX}$ & $\mathrm{TX}$ & Sorafenib & 27.39 & 87969.74 \\
\hline 170 & $\mathrm{DN}$ & $\mathrm{DN}$ & $\mathrm{TX}$ & $\mathrm{DN}$ & $\mathrm{TX}$ & $\mathrm{TX}$ & $\mathrm{DN}$ & 27.37 & 87936.21 \\
\hline 171 & $\mathrm{DN}$ & $\mathrm{DN}$ & $\mathrm{TX}$ & $\mathrm{DN}$ & $\mathrm{TX}$ & $\mathrm{DN}$ & Sorafenib & 25.38 & 87866.66 \\
\hline 172 & $\mathrm{DN}$ & $\mathrm{DN}$ & $\mathrm{TX}$ & $\mathrm{DN}$ & $\mathrm{TX}$ & $\mathrm{DN}$ & $\mathrm{DN}$ & 25.35 & 87833.12 \\
\hline 173 & $\mathrm{DN}$ & $\mathrm{DN}$ & $\mathrm{TX}$ & $\mathrm{DN}$ & $\mathrm{DN}$ & $\mathrm{TX}$ & Sorafenib & 15.55 & 47451.88 \\
\hline 174 & $\mathrm{DN}$ & $\mathrm{DN}$ & $\mathrm{TX}$ & $\mathrm{DN}$ & $\mathrm{DN}$ & $\mathrm{TX}$ & $\mathrm{DN}$ & 15.53 & 47418.34 \\
\hline 175 & $\mathrm{DN}$ & $\mathrm{DN}$ & $\mathrm{TX}$ & $\mathrm{DN}$ & $\mathrm{DN}$ & $\mathrm{DN}$ & Sorafenib & 13.54 & 47348.79 \\
\hline 176 & $\mathrm{DN}$ & $\mathrm{DN}$ & $\mathrm{TX}$ & $\mathrm{DN}$ & $\mathrm{DN}$ & $\mathrm{DN}$ & $\mathrm{DN}$ & 13.51 & 47315.26 \\
\hline 177 & $\mathrm{DN}$ & $\mathrm{DN}$ & $\mathrm{DN}$ & $\mathrm{TX}$ & $\mathrm{TX}$ & $\mathrm{TX}$ & Sorafenib & 61.95 & 206197.67 \\
\hline 178 & $\mathrm{DN}$ & $\mathrm{DN}$ & DN & $\mathrm{TX}$ & $\mathrm{TX}$ & $\mathrm{TX}$ & $\mathrm{DN}$ & 61.92 & 206164.13 \\
\hline 179 & $\mathrm{DN}$ & $\mathrm{DN}$ & DN & $\mathrm{TX}$ & $\mathrm{TX}$ & $\mathrm{DN}$ & Sorafenib & 59.93 & 206094.58 \\
\hline 180 & $\mathrm{DN}$ & $\mathrm{DN}$ & DN & $\mathrm{TX}$ & $\mathrm{TX}$ & $\mathrm{DN}$ & $\mathrm{DN}$ & 59.91 & 206061.05 \\
\hline 181 & $\mathrm{DN}$ & $\mathrm{DN}$ & DN & $\mathrm{TX}$ & $\mathrm{DN}$ & $\mathrm{TX}$ & Sorafenib & 50.11 & 165679.80 \\
\hline 182 & $\mathrm{DN}$ & DN & DN & $\mathrm{TX}$ & $\mathrm{DN}$ & $\mathrm{TX}$ & DN & 50.08 & 165646.27 \\
\hline 183 & $\mathrm{DN}$ & $\mathrm{DN}$ & DN & $\mathrm{TX}$ & $\mathrm{DN}$ & $\mathrm{DN}$ & Sorafenib & 48.09 & 165576.71 \\
\hline 184 & $\mathrm{DN}$ & $\mathrm{DN}$ & DN & $\mathrm{TX}$ & $\mathrm{DN}$ & $\mathrm{DN}$ & $\mathrm{DN}$ & 48.07 & 165543.18 \\
\hline 185 & $\mathrm{DN}$ & $\mathrm{DN}$ & DN & $\mathrm{DN}$ & $\mathrm{TX}$ & $\mathrm{TX}$ & Sorafenib & 13.88 & 41742.79 \\
\hline 186 & $\mathrm{DN}$ & $\mathrm{DN}$ & $\mathrm{DN}$ & $\mathrm{DN}$ & $\mathrm{TX}$ & $\mathrm{TX}$ & $\mathrm{DN}$ & 13.86 & 41709.26 \\
\hline
\end{tabular}




$\begin{array}{lllllllclll}187 & \text { DN } & \text { DN } & \text { DN } & \text { DN } & \text { TX } & \text { DN } & \text { Sorafenib } & 11.87 & 41639.71 & \text { X } \\ 188 & \text { DN } & \text { DN } & \text { DN } & \text { DN } & \text { TX } & \text { DN } & \text { DN } & 11.84 & 41606.17 & \text { X } \\ 189 & \text { DN } & \text { DN } & \text { DN } & \text { DN } & \text { DN } & \text { TX } & \text { Sorafenib } & 2.04 & 1224.92 & \\ 190 & \text { DN } & \text { DN } & \text { DN } & \text { DN } & \text { DN } & \text { TX } & \text { DN } & 2.02 & 1191.39 & \\ 191 & \text { DN } & \text { DN } & \text { DN } & \text { DN } & \text { DN } & \text { DN } & \text { Sorafenib } & 0.02 & 1121.84 & \text { X } \\ 192 & \text { DN } & \text { DN } & \text { DN } & \text { DN } & \text { DN } & \text { DN } & \text { DN } & 0.00 & 1088.30 & \end{array}$

Initial Calculated MELD [20,25)

\begin{tabular}{|c|c|c|c|c|c|c|c|c|c|c|}
\hline & & Mont & ecision & & & & & Dama & & \\
\hline & $\begin{array}{c}\text { Initial } \\
\text { Decision }\end{array}$ & M1 & M2 & M3 & M4 & M5 & Drop & Lives & Cost & P-O? \\
\hline 1 & TACE & $\mathrm{TX}$ & $\mathrm{TX}$ & $\mathrm{TX}$ & $\mathrm{TX}$ & $\mathrm{TX}$ & Sorafenib & 77.72 & 271121.30 & \\
\hline 2 & TACE & $\mathrm{TX}$ & $\mathrm{TX}$ & $\mathrm{TX}$ & $\mathrm{TX}$ & $\mathrm{TX}$ & $\mathrm{DN}$ & 77.68 & 271062.86 & \\
\hline 3 & TACE & $\mathrm{TX}$ & $\mathrm{TX}$ & $\mathrm{TX}$ & $\mathrm{TX}$ & TACE & Sorafenib & 75.69 & 253844.61 & $\mathrm{X}$ \\
\hline 4 & TACE & $\mathrm{TX}$ & $\mathrm{TX}$ & $\mathrm{TX}$ & $\mathrm{TX}$ & TACE & $\mathrm{DN}$ & 75.65 & 253786.16 & $\mathrm{X}$ \\
\hline 5 & TACE & $\mathrm{TX}$ & $\mathrm{TX}$ & $\mathrm{TX}$ & TACE & $\mathrm{TX}$ & Sorafenib & 61.08 & 129381.35 & $\mathrm{X}$ \\
\hline 6 & TACE & $\mathrm{TX}$ & $\mathrm{TX}$ & $\mathrm{TX}$ & TACE & $\mathrm{TX}$ & $\mathrm{DN}$ & 61.05 & 129322.90 & $\mathrm{X}$ \\
\hline 7 & TACE & $\mathrm{TX}$ & $\mathrm{TX}$ & $\mathrm{TX}$ & TACE & TACE & Sorafenib & 59.06 & 112104.65 & $\mathrm{X}$ \\
\hline 8 & TACE & $\mathrm{TX}$ & $\mathrm{TX}$ & $\mathrm{TX}$ & TACE & TACE & $\mathrm{DN}$ & 59.02 & 112046.21 & $\mathrm{X}$ \\
\hline 9 & TACE & $\mathrm{TX}$ & $\mathrm{TX}$ & TACE & $\mathrm{TX}$ & $\mathrm{TX}$ & Sorafenib & 70.82 & 212339.94 & \\
\hline 10 & TACE & $\mathrm{TX}$ & $\mathrm{TX}$ & TACE & $\mathrm{TX}$ & $\mathrm{TX}$ & $\mathrm{DN}$ & 70.78 & 212281.50 & \\
\hline 11 & TACE & $\mathrm{TX}$ & $\mathrm{TX}$ & TACE & $\mathrm{TX}$ & TACE & Sorafenib & 68.79 & 195063.24 & $\mathrm{X}$ \\
\hline 12 & TACE & $\mathrm{TX}$ & $\mathrm{TX}$ & TACE & $\mathrm{TX}$ & TACE & $\mathrm{DN}$ & 68.75 & 195004.80 & $\mathrm{X}$ \\
\hline 13 & TACE & $\mathrm{TX}$ & $\mathrm{TX}$ & TACE & TACE & $\mathrm{TX}$ & Sorafenib & 54.19 & 70599.99 & $\mathrm{X}$ \\
\hline 14 & TACE & $\mathrm{TX}$ & $\mathrm{TX}$ & TACE & TACE & $\mathrm{TX}$ & $\mathrm{DN}$ & 54.15 & 70541.54 & $\mathrm{X}$ \\
\hline 15 & TACE & $\mathrm{TX}$ & $\mathrm{TX}$ & TACE & TACE & TACE & Sorafenib & 52.16 & 53323.29 & $\mathrm{X}$ \\
\hline 16 & TACE & $\mathrm{TX}$ & $\mathrm{TX}$ & TACE & TACE & TACE & $\mathrm{DN}$ & 52.12 & 53264.84 & $\mathrm{X}$ \\
\hline 17 & TACE & $\mathrm{TX}$ & TACE & $\mathrm{TX}$ & $\mathrm{TX}$ & $\mathrm{TX}$ & Sorafenib & 74.88 & 246918.71 & $\mathrm{X}$ \\
\hline 18 & TACE & $\mathrm{TX}$ & TACE & $\mathrm{TX}$ & $\mathrm{TX}$ & $\mathrm{TX}$ & $\mathrm{DN}$ & 74.84 & 246860.26 & $\mathrm{X}$ \\
\hline 19 & TACE & $\mathrm{TX}$ & TACE & $\mathrm{TX}$ & $\mathrm{TX}$ & TACE & Sorafenib & 72.85 & 229642.01 & \\
\hline 20 & TACE & $\mathrm{TX}$ & TACE & $\mathrm{TX}$ & $\mathrm{TX}$ & TACE & $\mathrm{DN}$ & 72.81 & 229583.56 & \\
\hline 21 & TACE & $\mathrm{TX}$ & TACE & $\mathrm{TX}$ & TACE & $\mathrm{TX}$ & Sorafenib & 58.24 & 105178.75 & $\mathrm{X}$ \\
\hline 22 & TACE & $\mathrm{TX}$ & TACE & $\mathrm{TX}$ & TACE & $\mathrm{TX}$ & $\mathrm{DN}$ & 58.21 & 105120.31 & $\mathrm{X}$ \\
\hline 23 & TACE & $\mathrm{TX}$ & TACE & $\mathrm{TX}$ & TACE & TACE & Sorafenib & 56.22 & 87902.05 & $\mathrm{X}$ \\
\hline 24 & TACE & $\mathrm{TX}$ & TACE & $\mathrm{TX}$ & TACE & TACE & $\mathrm{DN}$ & 56.18 & 87843.61 & $\mathrm{X}$ \\
\hline 25 & TACE & $\mathrm{TX}$ & TACE & TACE & $\mathrm{TX}$ & $\mathrm{TX}$ & Sorafenib & 67.98 & 188137.34 & $\mathrm{X}$ \\
\hline 26 & TACE & $\mathrm{TX}$ & TACE & TACE & $\mathrm{TX}$ & $\mathrm{TX}$ & $\mathrm{DN}$ & 67.94 & 188078.90 & $\mathrm{X}$ \\
\hline 27 & TACE & $\mathrm{TX}$ & TACE & TACE & $\mathrm{TX}$ & TACE & Sorafenib & 65.95 & 170860.64 & $\mathrm{X}$ \\
\hline 28 & TACE & $\mathrm{TX}$ & TACE & TACE & $\mathrm{TX}$ & TACE & $\mathrm{DN}$ & 65.91 & 170802.20 & $\mathrm{X}$ \\
\hline 29 & TACE & $\mathrm{TX}$ & TACE & TACE & TACE & $\mathrm{TX}$ & Sorafenib & 51.35 & 46397.39 & $\mathrm{X}$ \\
\hline 30 & TACE & $\mathrm{TX}$ & TACE & TACE & TACE & $\mathrm{TX}$ & $\mathrm{DN}$ & 51.31 & 46338.94 & $X$ \\
\hline
\end{tabular}




\begin{tabular}{|c|c|c|c|c|c|c|c|c|c|}
\hline 31 & TACE & $\mathrm{TX}$ & TACE & TACE & TACE & TACE & Sorafenib & 49.32 & 29120.69 \\
\hline 32 & TACE & $\mathrm{TX}$ & TACE & TACE & TACE & TACE & $\mathrm{DN}$ & 49.28 & 29062.24 \\
\hline 33 & TACE & TACE & $\mathrm{TX}$ & $\mathrm{TX}$ & $\mathrm{TX}$ & $\mathrm{TX}$ & Sorafenib & 77.72 & 271121.30 \\
\hline 34 & TACE & TACE & $\mathrm{TX}$ & $\mathrm{TX}$ & $\mathrm{TX}$ & $\mathrm{TX}$ & $\mathrm{DN}$ & 77.68 & 271062.86 \\
\hline 35 & TACE & TACE & $\mathrm{TX}$ & $\mathrm{TX}$ & $\mathrm{TX}$ & TACE & Sorafenib & 75.69 & 253844.61 \\
\hline 36 & TACE & TACE & $\mathrm{TX}$ & $\mathrm{TX}$ & $\mathrm{TX}$ & TACE & $\mathrm{DN}$ & 75.65 & 253786.16 \\
\hline 37 & TACE & TACE & $\mathrm{TX}$ & $\mathrm{TX}$ & TACE & $\mathrm{TX}$ & Sorafenib & 61.08 & 129381.35 \\
\hline 38 & TACE & TACE & $\mathrm{TX}$ & $\mathrm{TX}$ & TACE & $\mathrm{TX}$ & DN & 61.05 & 129322.90 \\
\hline 39 & TACE & TACE & $\mathrm{TX}$ & TX & TACE & TACE & Sorafenib & 59.06 & 112104.65 \\
\hline 40 & TACE & TACE & $\mathrm{TX}$ & $\mathrm{TX}$ & TACE & TACE & DN & 59.02 & 112046.21 \\
\hline 41 & TACE & TACE & $\mathrm{TX}$ & TACE & $\mathrm{TX}$ & $\mathrm{TX}$ & Sorafenib & 70.82 & 212339.94 \\
\hline 42 & TACE & TACE & $\mathrm{TX}$ & TACE & $\mathrm{TX}$ & $\mathrm{TX}$ & $\mathrm{DN}$ & 70.78 & 212281.50 \\
\hline 43 & TACE & TACE & $\mathrm{TX}$ & TACE & $\mathrm{TX}$ & TACE & Sorafenib & 68.79 & 195063.24 \\
\hline 44 & TACE & TACE & $\mathrm{TX}$ & TACE & $\mathrm{TX}$ & TACE & $\mathrm{DN}$ & 68.75 & 195004.80 \\
\hline 45 & TACE & TACE & $\mathrm{TX}$ & TACE & TACE & $\mathrm{TX}$ & Sorafenib & 54.19 & 70599.99 \\
\hline 46 & TACE & TACE & $\mathrm{TX}$ & TACE & TACE & $\mathrm{TX}$ & DN & 54.15 & 70541.54 \\
\hline 47 & TACE & TACE & $\mathrm{TX}$ & TACE & TACE & TACE & Sorafenib & 52.16 & 53323.29 \\
\hline 48 & TACE & TACE & TX & TACE & TACE & TACE & $\mathrm{DN}$ & 52.12 & 53264.84 \\
\hline 49 & TACE & TACE & TACE & $\mathrm{TX}$ & $\mathrm{TX}$ & $\mathrm{TX}$ & Sorafenib & 74.88 & 246918.71 \\
\hline 50 & TACE & TACE & TACE & $\mathrm{TX}$ & $\mathrm{TX}$ & $\mathrm{TX}$ & $\mathrm{DN}$ & 74.84 & 246860.26 \\
\hline 51 & TACE & TACE & TACE & TX & $\mathrm{TX}$ & TACE & Sorafenib & 72.85 & 229642.01 \\
\hline 52 & TACE & TACE & TACE & $\mathrm{TX}$ & $\mathrm{TX}$ & TACE & DN & 72.81 & 229583.56 \\
\hline 53 & TACE & TACE & TACE & $\mathrm{TX}$ & TACE & $\mathrm{TX}$ & Sorafenib & 58.24 & 105178.75 \\
\hline 54 & TACE & TACE & TACE & $\mathrm{TX}$ & TACE & $\mathrm{TX}$ & DN & 58.21 & 105120.31 \\
\hline 55 & TACE & TACE & TACE & $\mathrm{TX}$ & TACE & TACE & Sorafenib & 56.22 & 87902.05 \\
\hline 56 & TACE & TACE & TACE & $\mathrm{TX}$ & TACE & TACE & DN & 56.18 & 87843.61 \\
\hline 57 & TACE & TACE & TACE & TACE & $\mathrm{TX}$ & $\mathrm{TX}$ & Sorafenib & 67.98 & 188137.34 \\
\hline 58 & TACE & TACE & TACE & TACE & $\mathrm{TX}$ & $\mathrm{TX}$ & DN & 67.94 & 188078.90 \\
\hline 59 & TACE & TACE & TACE & TACE & $\mathrm{TX}$ & TACE & Sorafenib & 65.95 & 170860.64 \\
\hline 60 & TACE & TACE & TACE & TACE & $\mathrm{TX}$ & TACE & $\mathrm{DN}$ & 65.91 & 170802.20 \\
\hline 61 & TACE & TACE & TACE & TACE & TACE & $\mathrm{TX}$ & Sorafenib & 51.35 & 46397.39 \\
\hline 62 & TACE & TACE & TACE & TACE & TACE & $\mathrm{TX}$ & DN & 51.31 & 46338.94 \\
\hline 63 & TACE & TACE & TACE & TACE & TACE & TACE & Sorafenib & 49.32 & 29120.69 \\
\hline 64 & TACE & TACE & TACE & TACE & TACE & TACE & DN & 49.28 & 29062.24 \\
\hline 65 & RFA & $\mathrm{TX}$ & TX & $\mathrm{TX}$ & $\mathrm{TX}$ & TX & Sorafenib & 77.48 & 266347.78 \\
\hline 66 & RFA & $\mathrm{TX}$ & $\mathrm{TX}$ & $\mathrm{TX}$ & $\mathrm{TX}$ & $\mathrm{TX}$ & DN & 77.46 & 266307.25 \\
\hline 67 & RFA & $\mathrm{TX}$ & $\mathrm{TX}$ & $\mathrm{TX}$ & $\mathrm{TX}$ & RFA & Sorafenib & 77.48 & 266347.78 \\
\hline 68 & RFA & $\mathrm{TX}$ & $\mathrm{TX}$ & $\mathrm{TX}$ & $\mathrm{TX}$ & RFA & $\mathrm{DN}$ & 77.46 & 266307.25 \\
\hline 69 & RFA & TX & $\mathrm{TX}$ & $\mathrm{TX}$ & RFA & $\mathrm{TX}$ & Sorafenib & 70.36 & 162008.91 \\
\hline 70 & RFA & $\mathrm{TX}$ & $\mathrm{TX}$ & $\mathrm{TX}$ & RFA & $\mathrm{TX}$ & DN & 70.33 & 161968.38 \\
\hline 71 & RFA & $\mathrm{TX}$ & $\mathrm{TX}$ & $\mathrm{TX}$ & RFA & RFA & Sorafenib & 70.36 & 162008.91 \\
\hline 72 & RFA & $\mathrm{TX}$ & $\mathrm{TX}$ & $\mathrm{TX}$ & RFA & RFA & DN & 70.33 & 161968.38 \\
\hline 73 & RFA & $\mathrm{TX}$ & $\mathrm{TX}$ & RFA & $\mathrm{TX}$ & $\mathrm{TX}$ & Sorafenib & 69.06 & 143031.13 \\
\hline 74 & RFA & $\mathrm{TX}$ & $\mathrm{TX}$ & RFA & $\mathrm{TX}$ & $\mathrm{TX}$ & DN & 69.04 & 142990.60 \\
\hline 75 & RFA & $\mathrm{TX}$ & $\mathrm{TX}$ & RFA & $\mathrm{TX}$ & RFA & Sorafenib & 69.06 & 143031.13 \\
\hline 76 & RFA & $\mathrm{TX}$ & $\mathrm{TX}$ & RFA & $\mathrm{TX}$ & RFA & $\mathrm{DN}$ & 69.04 & 142990.60 \\
\hline
\end{tabular}




\begin{tabular}{|c|c|c|c|c|c|c|c|c|c|c|}
\hline 77 & RFA & $\mathrm{TX}$ & $\mathrm{TX}$ & RFA & RFA & $\mathrm{TX}$ & Sorafenib & 61.94 & 38692.26 & \\
\hline 78 & RFA & $\mathrm{TX}$ & TX & RFA & RFA & TX & DN & 61.92 & 38651.73 & \\
\hline 79 & RFA & $\mathrm{TX}$ & $\mathrm{TX}$ & RFA & RFA & RFA & Sorafenib & 61.94 & 38692.26 & $\mathrm{X}$ \\
\hline 80 & RFA & $\mathrm{TX}$ & $\mathrm{TX}$ & RFA & RFA & RFA & DN & 61.92 & 38651.73 & $\mathrm{X}$ \\
\hline 81 & RFA & $\mathrm{TX}$ & RFA & $\mathrm{TX}$ & $\mathrm{TX}$ & $\mathrm{TX}$ & Sorafenib & 76.19 & 247370.00 & $\mathrm{X}$ \\
\hline 82 & RFA & $\mathrm{TX}$ & RFA & $\mathrm{TX}$ & $\mathrm{TX}$ & $\mathrm{TX}$ & DN & 76.16 & 247329.46 & $\mathrm{X}$ \\
\hline 83 & RFA & $\mathrm{TX}$ & RFA & TX & $\mathrm{TX}$ & RFA & Sorafenib & 76.19 & 247370.00 & $\mathrm{X}$ \\
\hline 84 & RFA & $\mathrm{TX}$ & RFA & $\mathrm{TX}$ & $\mathrm{TX}$ & RFA & DN & 76.16 & 247329.46 & $\mathrm{X}$ \\
\hline 85 & RFA & $\mathrm{TX}$ & RFA & $\mathrm{TX}$ & RFA & $\mathrm{TX}$ & Sorafenib & 69.06 & 143031.13 & $\mathrm{X}$ \\
\hline 86 & RFA & $\mathrm{TX}$ & RFA & $\mathrm{TX}$ & RFA & $\mathrm{TX}$ & DN & 69.04 & 142990.60 & $\mathrm{X}$ \\
\hline 87 & RFA & $\mathrm{TX}$ & RFA & $\mathrm{TX}$ & RFA & RFA & Sorafenib & 69.06 & 143031.13 & $\mathrm{X}$ \\
\hline 88 & RFA & $\mathrm{TX}$ & RFA & $\mathrm{TX}$ & RFA & RFA & DN & 69.04 & 142990.60 & $\mathrm{X}$ \\
\hline 89 & RFA & $\mathrm{TX}$ & RFA & RFA & $\mathrm{TX}$ & $\mathrm{TX}$ & Sorafenib & 67.77 & 124053.35 & \\
\hline 90 & RFA & $\mathrm{TX}$ & RFA & RFA & $\mathrm{TX}$ & TX & DN & 67.74 & 124012.81 & \\
\hline 91 & RFA & $\mathrm{TX}$ & RFA & RFA & $\mathrm{TX}$ & RFA & Sorafenib & 67.77 & 124053.35 & $\mathrm{X}$ \\
\hline 92 & RFA & $\mathrm{TX}$ & RFA & RFA & $\mathrm{TX}$ & RFA & DN & 67.74 & 124012.81 & $\mathrm{X}$ \\
\hline 93 & RFA & $\mathrm{TX}$ & RFA & RFA & RFA & $\mathrm{TX}$ & Sorafenib & 60.65 & 19714.48 & \\
\hline 94 & RFA & $\mathrm{TX}$ & RFA & RFA & RFA & TX & DN & 60.62 & 19673.95 & \\
\hline 95 & RFA & $\mathrm{TX}$ & RFA & RFA & RFA & RFA & Sorafenib & 60.65 & 19714.48 & $\mathrm{X}$ \\
\hline 96 & RFA & $\mathrm{TX}$ & RFA & RFA & RFA & RFA & DN & 60.62 & 19673.95 & \\
\hline 97 & RFA & RFA & $\mathrm{TX}$ & $\mathrm{TX}$ & $\mathrm{TX}$ & $\mathrm{TX}$ & Sorafenib & 77.48 & 266347.78 & $\mathrm{X}$ \\
\hline 98 & RFA & RFA & $\mathrm{TX}$ & $\mathrm{TX}$ & $\mathrm{TX}$ & TX & DN & 77.46 & 266307.25 & $\mathrm{X}$ \\
\hline 99 & RFA & RFA & $\mathrm{TX}$ & $\mathrm{TX}$ & $\mathrm{TX}$ & RFA & Sorafenib & 77.48 & 266347.78 & $\mathrm{X}$ \\
\hline 100 & RFA & RFA & $\mathrm{TX}$ & $\mathrm{TX}$ & $\mathrm{TX}$ & RFA & DN & 77.46 & 266307.25 & $\mathrm{X}$ \\
\hline 101 & RFA & RFA & $\mathrm{TX}$ & $\mathrm{TX}$ & RFA & $\mathrm{TX}$ & Sorafenib & 70.36 & 162008.91 & $\mathrm{X}$ \\
\hline 102 & RFA & RFA & $\mathrm{TX}$ & $\mathrm{TX}$ & RFA & $\mathrm{TX}$ & DN & 70.33 & 161968.38 & $\mathrm{X}$ \\
\hline 103 & RFA & RFA & $\mathrm{TX}$ & $\mathrm{TX}$ & RFA & RFA & Sorafenib & 70.36 & 162008.91 & $\mathrm{X}$ \\
\hline 104 & RFA & RFA & $\mathrm{TX}$ & $\mathrm{TX}$ & RFA & RFA & $\mathrm{DN}$ & 70.33 & 161968.38 & $\mathrm{X}$ \\
\hline 105 & RFA & RFA & $\mathrm{TX}$ & RFA & $\mathrm{TX}$ & $\mathrm{TX}$ & Sorafenib & 69.06 & 143031.13 & $\mathrm{X}$ \\
\hline 106 & RFA & RFA & $\mathrm{TX}$ & RFA & $\mathrm{TX}$ & $\mathrm{TX}$ & $\mathrm{DN}$ & 69.04 & 142990.60 & $\mathrm{X}$ \\
\hline 107 & RFA & RFA & $\mathrm{TX}$ & RFA & $\mathrm{TX}$ & RFA & Sorafenib & 69.06 & 143031.13 & $\mathrm{X}$ \\
\hline 108 & RFA & RFA & $\mathrm{TX}$ & RFA & $\mathrm{TX}$ & RFA & DN & 69.04 & 142990.60 & $\mathrm{X}$ \\
\hline 109 & RFA & RFA & $\mathrm{TX}$ & RFA & RFA & TX & Sorafenib & 61.94 & 38692.26 & $\mathrm{X}$ \\
\hline 110 & RFA & RFA & $\mathrm{TX}$ & RFA & RFA & TX & DN & 61.92 & 38651.73 & $\mathrm{X}$ \\
\hline 111 & RFA & RFA & $\mathrm{TX}$ & RFA & RFA & RFA & Sorafenib & 61.94 & 38692.26 & $\mathrm{X}$ \\
\hline 112 & RFA & RFA & $\mathrm{TX}$ & RFA & RFA & RFA & DN & 61.92 & 38651.73 & $\mathrm{X}$ \\
\hline 113 & RFA & RFA & RFA & $\mathrm{TX}$ & $\mathrm{TX}$ & $\mathrm{TX}$ & Sorafenib & 76.19 & 247370.00 & $\mathrm{X}$ \\
\hline 114 & RFA & RFA & RFA & $\mathrm{TX}$ & $\mathrm{TX}$ & $\mathrm{TX}$ & $\mathrm{DN}$ & 76.16 & 247329.46 & $\mathrm{X}$ \\
\hline 115 & RFA & RFA & RFA & $\mathrm{TX}$ & $\mathrm{TX}$ & RFA & Sorafenib & 76.19 & 247370.00 & $\mathrm{X}$ \\
\hline 116 & RFA & RFA & RFA & $\mathrm{TX}$ & $\mathrm{TX}$ & RFA & DN & 76.16 & 247329.46 & $\mathrm{X}$ \\
\hline 117 & RFA & RFA & RFA & $\mathrm{TX}$ & RFA & TX & Sorafenib & 69.06 & 143031.13 & $\mathrm{X}$ \\
\hline 118 & RFA & RFA & RFA & $\mathrm{TX}$ & RFA & $\mathrm{TX}$ & DN & 69.04 & 142990.60 & $\mathrm{X}$ \\
\hline 119 & RFA & RFA & RFA & $\mathrm{TX}$ & RFA & RFA & Sorafenib & 69.06 & 143031.13 & $\mathrm{X}$ \\
\hline 120 & RFA & RFA & RFA & $\mathrm{TX}$ & RFA & RFA & DN & 69.04 & 142990.60 & $\mathrm{X}$ \\
\hline 121 & RFA & RFA & RFA & RFA & $\mathrm{TX}$ & TX & Sorafenib & 67.77 & 124053.35 & $\mathrm{X}$ \\
\hline 122 & RFA & RFA & RFA & RFA & TX & TX & DN & 67.74 & 124012.81 & $\mathrm{X}$ \\
\hline
\end{tabular}




\begin{tabular}{|c|c|c|c|c|c|c|c|c|c|}
\hline 123 & RFA & RFA & RFA & RFA & TX & RFA & Sorafenib & 67.77 & 124053.35 \\
\hline 124 & RFA & RFA & RFA & RFA & $\mathrm{TX}$ & RFA & $\mathrm{DN}$ & 67.74 & 124012.81 \\
\hline 125 & RFA & RFA & RFA & RFA & RFA & $\mathrm{TX}$ & Sorafenib & 60.65 & 19714.48 \\
\hline 126 & RFA & RFA & RFA & RFA & RFA & $\mathrm{TX}$ & $\mathrm{DN}$ & 60.62 & 19673.95 \\
\hline 127 & RFA & RFA & RFA & RFA & RFA & RFA & Sorafenib & 60.65 & 19714.48 \\
\hline 128 & RFA & RFA & RFA & RFA & RFA & RFA & $\mathrm{DN}$ & 60.62 & 19673.95 \\
\hline 129 & DN & $\mathrm{TX}$ & $\mathrm{TX}$ & $\mathrm{TX}$ & $\mathrm{TX}$ & $\mathrm{TX}$ & Sorafenib & 76.24 & 243892.05 \\
\hline 130 & $\mathrm{DN}$ & $\mathrm{TX}$ & $\mathrm{TX}$ & $\mathrm{TX}$ & TX & $\mathrm{TX}$ & $\mathrm{DN}$ & 76.21 & 243858.52 \\
\hline 131 & $\mathrm{DN}$ & $\mathrm{TX}$ & $\mathrm{TX}$ & $\mathrm{TX}$ & $\mathrm{TX}$ & DN & Sorafenib & 70.90 & 243619.22 \\
\hline 132 & DN & $\mathrm{TX}$ & $\mathrm{TX}$ & $\mathrm{TX}$ & $\mathrm{TX}$ & $\mathrm{DN}$ & DN & 70.88 & 243585.69 \\
\hline 133 & $\mathrm{DN}$ & $\mathrm{TX}$ & $\mathrm{TX}$ & $\mathrm{TX}$ & DN & $\mathrm{TX}$ & Sorafenib & 33.03 & 96065.57 \\
\hline 134 & DN & $\mathrm{TX}$ & $\mathrm{TX}$ & $\mathrm{TX}$ & DN & $\mathrm{TX}$ & $\mathrm{DN}$ & 33.01 & 96032.04 \\
\hline 135 & $\mathrm{DN}$ & $\mathrm{TX}$ & $\mathrm{TX}$ & TX & DN & $\mathrm{DN}$ & Sorafenib & 27.69 & 95792.75 \\
\hline 136 & $\mathrm{DN}$ & $\mathrm{TX}$ & $\mathrm{TX}$ & TX & $\mathrm{DN}$ & $\mathrm{DN}$ & $\mathrm{DN}$ & 27.67 & 95759.21 \\
\hline 137 & $\mathrm{DN}$ & $\mathrm{TX}$ & $\mathrm{TX}$ & $\mathrm{DN}$ & TX & $\mathrm{TX}$ & Sorafenib & 54.39 & 169147.39 \\
\hline 138 & $\mathrm{DN}$ & $\mathrm{TX}$ & $\mathrm{TX}$ & DN & $\mathrm{TX}$ & $\mathrm{TX}$ & $\mathrm{DN}$ & 54.37 & 169113.86 \\
\hline 139 & $\mathrm{DN}$ & $\mathrm{TX}$ & $\mathrm{TX}$ & DN & $\mathrm{TX}$ & DN & Sorafenib & 49.05 & 168874.57 \\
\hline 140 & $\mathrm{DN}$ & $\mathrm{TX}$ & $\mathrm{TX}$ & $\mathrm{DN}$ & $\mathrm{TX}$ & DN & DN & 49.03 & 168841.03 \\
\hline 141 & $\mathrm{DN}$ & $\mathrm{TX}$ & $\mathrm{TX}$ & $\mathrm{DN}$ & DN & $\mathrm{TX}$ & Sorafenib & 11.19 & 21320.92 \\
\hline 142 & $\mathrm{DN}$ & $\mathrm{TX}$ & $\mathrm{TX}$ & $\mathrm{DN}$ & DN & $\mathrm{TX}$ & $\mathrm{DN}$ & 11.16 & 21287.38 \\
\hline 143 & DN & $\mathrm{TX}$ & $\mathrm{TX}$ & DN & DN & DN & Sorafenib & 5.85 & 21048.09 \\
\hline 144 & DN & $\mathrm{TX}$ & $\mathrm{TX}$ & $\mathrm{DN}$ & DN & $\mathrm{DN}$ & DN & 5.82 & 21014.56 \\
\hline 145 & $\mathrm{DN}$ & $\mathrm{TX}$ & DN & $\mathrm{TX}$ & $\mathrm{TX}$ & $\mathrm{TX}$ & Sorafenib & 70.90 & 225628.52 \\
\hline 146 & $\mathrm{DN}$ & $\mathrm{TX}$ & DN & $\mathrm{TX}$ & $\mathrm{TX}$ & $\mathrm{TX}$ & $\mathrm{DN}$ & 70.88 & 225594.99 \\
\hline 147 & $\mathrm{DN}$ & $\mathrm{TX}$ & DN & $\mathrm{TX}$ & $\mathrm{TX}$ & DN & Sorafenib & 65.56 & 225355.70 \\
\hline 148 & $\mathrm{DN}$ & $\mathrm{TX}$ & $\mathrm{DN}$ & $\mathrm{TX}$ & $\mathrm{TX}$ & $\mathrm{DN}$ & $\mathrm{DN}$ & 65.54 & 225322.16 \\
\hline 149 & $\mathrm{DN}$ & $\mathrm{TX}$ & $\mathrm{DN}$ & $\mathrm{TX}$ & DN & $\mathrm{TX}$ & Sorafenib & 27.69 & 77802.05 \\
\hline 150 & DN & $\mathrm{TX}$ & $\mathrm{DN}$ & $\mathrm{TX}$ & DN & $\mathrm{TX}$ & $\mathrm{DN}$ & 27.67 & 77768.51 \\
\hline 151 & $\mathrm{DN}$ & $\mathrm{TX}$ & $\mathrm{DN}$ & $\mathrm{TX}$ & DN & $\mathrm{DN}$ & Sorafenib & 22.36 & 77529.22 \\
\hline 152 & $\mathrm{DN}$ & $\mathrm{TX}$ & $\mathrm{DN}$ & $\mathrm{TX}$ & DN & $\mathrm{DN}$ & $\mathrm{DN}$ & 22.33 & 77495.69 \\
\hline 153 & $\mathrm{DN}$ & $\mathrm{TX}$ & DN & DN & $\mathrm{TX}$ & $\mathrm{TX}$ & Sorafenib & 49.05 & 150883.87 \\
\hline 154 & $\mathrm{DN}$ & $\mathrm{TX}$ & DN & $\mathrm{DN}$ & $\mathrm{TX}$ & $\mathrm{TX}$ & DN & 49.03 & 150850.33 \\
\hline 155 & $\mathrm{DN}$ & $\mathrm{TX}$ & $\mathrm{DN}$ & $\mathrm{DN}$ & $\mathrm{TX}$ & DN & Sorafenib & 43.72 & 150611.04 \\
\hline 156 & $\mathrm{DN}$ & $\mathrm{TX}$ & $\mathrm{DN}$ & DN & $\mathrm{TX}$ & $\mathrm{DN}$ & $\mathrm{DN}$ & 43.69 & 150577.51 \\
\hline 157 & $\mathrm{DN}$ & TX & DN & $\mathrm{DN}$ & DN & $\mathrm{TX}$ & Sorafenib & 5.85 & 3057.39 \\
\hline 158 & $\mathrm{DN}$ & $\mathrm{TX}$ & DN & $\mathrm{DN}$ & DN & $\mathrm{TX}$ & $\mathrm{DN}$ & 5.82 & 3023.86 \\
\hline 159 & $\mathrm{DN}$ & $\mathrm{TX}$ & $\mathrm{DN}$ & DN & DN & $\mathrm{DN}$ & Sorafenib & 0.51 & 2784.56 \\
\hline 160 & $\mathrm{DN}$ & $\mathrm{TX}$ & DN & $\mathrm{DN}$ & DN & DN & $\mathrm{DN}$ & 0.49 & 2751.03 \\
\hline 161 & $\mathrm{DN}$ & $\mathrm{DN}$ & $\mathrm{TX}$ & $\mathrm{TX}$ & $\mathrm{TX}$ & $\mathrm{TX}$ & Sorafenib & 75.75 & 242229.21 \\
\hline 162 & $\mathrm{DN}$ & DN & TX & $\mathrm{TX}$ & TX & $\mathrm{TX}$ & $\mathrm{DN}$ & 75.73 & 242195.68 \\
\hline 163 & $\mathrm{DN}$ & $\mathrm{DN}$ & $\mathrm{TX}$ & $\mathrm{TX}$ & $\mathrm{TX}$ & DN & Sorafenib & 70.41 & 241956.38 \\
\hline 164 & $\mathrm{DN}$ & DN & $\mathrm{TX}$ & $\mathrm{TX}$ & TX & DN & $\mathrm{DN}$ & 70.39 & 241922.85 \\
\hline 165 & $\mathrm{DN}$ & $\mathrm{DN}$ & $\mathrm{TX}$ & $\mathrm{TX}$ & DN & $\mathrm{TX}$ & Sorafenib & 32.55 & 94402.73 \\
\hline 166 & $\mathrm{DN}$ & $\mathrm{DN}$ & $\mathrm{TX}$ & $\mathrm{TX}$ & DN & $\mathrm{TX}$ & $\mathrm{DN}$ & 32.52 & 94369.20 \\
\hline 167 & $\mathrm{DN}$ & DN & TX & $\mathrm{TX}$ & DN & $\mathrm{DN}$ & Sorafenib & 27.21 & 94129.91 \\
\hline 168 & $\mathrm{DN}$ & $\mathrm{DN}$ & $\mathrm{TX}$ & TX & $\mathrm{DN}$ & $\mathrm{DN}$ & $\mathrm{DN}$ & 27.18 & 94096.37 \\
\hline
\end{tabular}




\begin{tabular}{|c|c|c|c|c|c|c|c|c|c|c|}
\hline 169 & DN & $\mathrm{DN}$ & $\mathrm{TX}$ & $\mathrm{DN}$ & $\mathrm{TX}$ & $\mathrm{TX}$ & Sorafenib & 53.91 & 167484.55 & $\mathrm{X}$ \\
\hline 170 & $\mathrm{DN}$ & $\mathrm{DN}$ & $\mathrm{TX}$ & DN & $\mathrm{TX}$ & $\mathrm{TX}$ & DN & 53.88 & 167451.02 & $\mathrm{X}$ \\
\hline 171 & $\mathrm{DN}$ & $\mathrm{DN}$ & $\mathrm{TX}$ & DN & $\mathrm{TX}$ & $\mathrm{DN}$ & Sorafenib & 48.57 & 167211.73 & $\mathrm{X}$ \\
\hline 172 & DN & $\mathrm{DN}$ & $\mathrm{TX}$ & $\mathrm{DN}$ & $\mathrm{TX}$ & DN & DN & 48.54 & 167178.19 & $\mathrm{X}$ \\
\hline 173 & DN & $\mathrm{DN}$ & $\mathrm{TX}$ & DN & $\mathrm{DN}$ & $\mathrm{TX}$ & Sorafenib & 10.70 & 19658.08 & \\
\hline 174 & DN & $\mathrm{DN}$ & $\mathrm{TX}$ & $\mathrm{DN}$ & $\mathrm{DN}$ & $\mathrm{TX}$ & DN & 10.68 & 19624.54 & \\
\hline 175 & DN & $\mathrm{DN}$ & $\mathrm{TX}$ & $\mathrm{DN}$ & $\mathrm{DN}$ & $\mathrm{DN}$ & Sorafenib & 5.36 & 19385.25 & $\mathrm{X}$ \\
\hline 176 & $\mathrm{DN}$ & $\mathrm{DN}$ & $\mathrm{TX}$ & DN & $\mathrm{DN}$ & $\mathrm{DN}$ & $\mathrm{DN}$ & 5.34 & 19351.72 & $\mathrm{X}$ \\
\hline 177 & $\mathrm{DN}$ & $\mathrm{DN}$ & $\mathrm{DN}$ & TX & $\mathrm{TX}$ & $\mathrm{TX}$ & Sorafenib & 70.41 & 223965.68 & $\mathrm{X}$ \\
\hline 178 & DN & $\mathrm{DN}$ & $\mathrm{DN}$ & $\mathrm{TX}$ & $\mathrm{TX}$ & $\mathrm{TX}$ & DN & 70.39 & 223932.15 & $\mathrm{X}$ \\
\hline 179 & $\mathrm{DN}$ & $\mathrm{DN}$ & $\mathrm{DN}$ & TX & $\mathrm{TX}$ & $\mathrm{DN}$ & Sorafenib & 65.08 & 223692.86 & $\mathrm{X}$ \\
\hline 180 & $\mathrm{DN}$ & $\mathrm{DN}$ & $\mathrm{DN}$ & $\mathrm{TX}$ & $\mathrm{TX}$ & DN & DN & 65.05 & 223659.32 & $\mathrm{X}$ \\
\hline 181 & $\mathrm{DN}$ & $\mathrm{DN}$ & $\mathrm{DN}$ & $\mathrm{TX}$ & $\mathrm{DN}$ & $\mathrm{TX}$ & Sorafenib & 27.21 & 76139.21 & $\mathrm{X}$ \\
\hline 182 & DN & $\mathrm{DN}$ & $\mathrm{DN}$ & $\mathrm{TX}$ & $\mathrm{DN}$ & $\mathrm{TX}$ & $\mathrm{DN}$ & 27.18 & 76105.67 & $\mathrm{X}$ \\
\hline 183 & $\mathrm{DN}$ & $\mathrm{DN}$ & $\mathrm{DN}$ & $\mathrm{TX}$ & $\mathrm{DN}$ & $\mathrm{DN}$ & Sorafenib & 21.87 & 75866.38 & $\mathrm{X}$ \\
\hline 184 & $\mathrm{DN}$ & $\mathrm{DN}$ & $\mathrm{DN}$ & $\mathrm{TX}$ & $\mathrm{DN}$ & $\mathrm{DN}$ & $\mathrm{DN}$ & 21.85 & 75832.85 & $\mathrm{X}$ \\
\hline 185 & $\mathrm{DN}$ & $\mathrm{DN}$ & DN & $\mathrm{DN}$ & $\mathrm{TX}$ & $\mathrm{TX}$ & Sorafenib & 48.57 & 149221.03 & $\mathrm{X}$ \\
\hline 186 & $\mathrm{DN}$ & $\mathrm{DN}$ & $\mathrm{DN}$ & DN & $\mathrm{TX}$ & $\mathrm{TX}$ & DN & 48.54 & 149187.49 & $\mathrm{X}$ \\
\hline 187 & DN & $\mathrm{DN}$ & DN & DN & $\mathrm{TX}$ & DN & Sorafenib & 43.23 & 148948.20 & $\mathrm{X}$ \\
\hline 188 & DN & $\mathrm{DN}$ & $\mathrm{DN}$ & DN & $\mathrm{TX}$ & $\mathrm{DN}$ & $\mathrm{DN}$ & 43.21 & 148914.67 & $\mathrm{X}$ \\
\hline 189 & $\mathrm{DN}$ & $\mathrm{DN}$ & $\mathrm{DN}$ & DN & $\mathrm{DN}$ & $\mathrm{TX}$ & Sorafenib & 5.36 & 1394.55 & \\
\hline 190 & DN & $\mathrm{DN}$ & $\mathrm{DN}$ & $\mathrm{DN}$ & $\mathrm{DN}$ & $\mathrm{TX}$ & $\mathrm{DN}$ & 5.34 & 1361.02 & \\
\hline 191 & $\mathrm{DN}$ & $\mathrm{DN}$ & $\mathrm{DN}$ & DN & $\mathrm{DN}$ & $\mathrm{DN}$ & Sorafenib & 0.02 & 1121.72 & \\
\hline 192 & $\mathrm{DN}$ & $\mathrm{DN}$ & $\mathrm{DN}$ & $\mathrm{DN}$ & $\mathrm{DN}$ & $\mathrm{DN}$ & $\mathrm{DN}$ & 0.00 & 1088.19 & \\
\hline
\end{tabular}

\section{Initial Calculated MELD $\geq 25$}

\begin{tabular}{|c|c|c|c|c|c|c|c|c|c|c|}
\hline & & Montl & cision & & & & & Dame & & \\
\hline & $\begin{array}{c}\text { Initial } \\
\text { Decision }\end{array}$ & M1 & M2 & M3 & M4 & M5 & Drop & Lives & Cost & Pareto? \\
\hline 1 & TACE & $\mathrm{TX}$ & $\mathrm{TX}$ & $\mathrm{TX}$ & $\mathrm{TX}$ & $\mathrm{TX}$ & Sorafenib & 77.71 & 271092.90 & \\
\hline 2 & TACE & $\mathrm{TX}$ & $\mathrm{TX}$ & $\mathrm{TX}$ & $\mathrm{TX}$ & $\mathrm{TX}$ & DN & 77.67 & 271034.45 & \\
\hline 3 & TACE & $\mathrm{TX}$ & $\mathrm{TX}$ & $\mathrm{TX}$ & TX & TACE & Sorafenib & 77.71 & 271092.90 & \\
\hline 4 & TACE & $\mathrm{TX}$ & $\mathrm{TX}$ & $\mathrm{TX}$ & $\mathrm{TX}$ & TACE & DN & 77.67 & 271034.45 & \\
\hline 5 & TACE & $\mathrm{TX}$ & $\mathrm{TX}$ & $\mathrm{TX}$ & TACE & $\mathrm{TX}$ & Sorafenib & 69.60 & 201960.74 & $\mathrm{X}$ \\
\hline 6 & TACE & $\mathrm{TX}$ & $\mathrm{TX}$ & $\mathrm{TX}$ & TACE & $\mathrm{TX}$ & DN & 69.56 & 201902.29 & $\mathrm{X}$ \\
\hline 7 & TACE & $\mathrm{TX}$ & $\mathrm{TX}$ & $\mathrm{TX}$ & TACE & TACE & Sorafenib & 69.60 & 201960.74 & $\mathrm{X}$ \\
\hline 8 & TACE & $\mathrm{TX}$ & $\mathrm{TX}$ & $\mathrm{TX}$ & TACE & TACE & DN & 69.56 & 201902.29 & $\mathrm{X}$ \\
\hline 9 & TACE & $\mathrm{TX}$ & $\mathrm{TX}$ & TACE & TX & TX & Sorafenib & 65.54 & 167381.97 & \\
\hline 10 & TACE & $\mathrm{TX}$ & $\mathrm{TX}$ & TACE & $\mathrm{TX}$ & $\mathrm{TX}$ & $\mathrm{DN}$ & 65.50 & 167323.53 & \\
\hline 11 & TACE & $\mathrm{TX}$ & $\mathrm{TX}$ & TACE & TX & TACE & Sorafenib & 65.54 & 167381.97 & \\
\hline 12 & TACE & $\mathrm{TX}$ & $\mathrm{TX}$ & TACE & $\mathrm{TX}$ & TACE & DN & 65.50 & 167323.53 & \\
\hline
\end{tabular}




\begin{tabular}{|c|c|c|c|c|c|c|c|c|c|}
\hline 13 & TACE & $\mathrm{TX}$ & $\mathrm{TX}$ & TACE & TACE & $\mathrm{TX}$ & Sorafenib & 57.43 & 98249.81 \\
\hline 14 & TACE & $\mathrm{TX}$ & $\mathrm{TX}$ & TACE & TACE & TX & $\mathrm{DN}$ & 57.39 & 98191.37 \\
\hline 15 & TACE & $\mathrm{TX}$ & $\mathrm{TX}$ & TACE & TACE & TACE & Sorafenib & 57.43 & 98249.81 \\
\hline 16 & TACE & $\mathrm{TX}$ & $\mathrm{TX}$ & TACE & TACE & TACE & $\mathrm{DN}$ & 57.39 & 98191.37 \\
\hline 17 & TACE & $\mathrm{TX}$ & TACE & $\mathrm{TX}$ & $\mathrm{TX}$ & $\mathrm{TX}$ & Sorafenib & 69.60 & 201960.74 \\
\hline 18 & TACE & $\mathrm{TX}$ & TACE & $\mathrm{TX}$ & $\mathrm{TX}$ & $\mathrm{TX}$ & DN & 69.56 & 201902.29 \\
\hline 19 & TACE & $\mathrm{TX}$ & TACE & $\mathrm{TX}$ & $\mathrm{TX}$ & TACE & Sorafenib & 69.60 & 201960.74 \\
\hline 20 & TACE & $\mathrm{TX}$ & TACE & $\mathrm{TX}$ & $\mathrm{TX}$ & TACE & $\mathrm{DN}$ & 69.56 & 201902.29 \\
\hline 21 & TACE & $\mathrm{TX}$ & TACE & $\mathrm{TX}$ & TACE & $\mathrm{TX}$ & Sorafenib & 61.48 & 132828.58 \\
\hline 22 & TACE & $\mathrm{TX}$ & TACE & $\mathrm{TX}$ & TACE & $\mathrm{TX}$ & DN & 61.45 & 132770.13 \\
\hline 23 & TACE & $\mathrm{TX}$ & TACE & $\mathrm{TX}$ & TACE & TACE & Sorafenib & 61.48 & 132828.58 \\
\hline 24 & TACE & $\mathrm{TX}$ & TACE & $\mathrm{TX}$ & TACE & TACE & DN & 61.45 & 132770.13 \\
\hline 25 & TACE & $\mathrm{TX}$ & TACE & TACE & $\mathrm{TX}$ & $\mathrm{TX}$ & Sorafenib & 57.43 & 98249.81 \\
\hline 26 & TACE & $\mathrm{TX}$ & TACE & TACE & $\mathrm{TX}$ & $\mathrm{TX}$ & DN & 57.39 & 98191.37 \\
\hline 27 & TACE & $\mathrm{TX}$ & TACE & TACE & $\mathrm{TX}$ & TACE & Sorafenib & 57.43 & 98249.81 \\
\hline 28 & TACE & $\mathrm{TX}$ & TACE & TACE & $\mathrm{TX}$ & TACE & DN & 57.39 & 98191.37 \\
\hline 29 & TACE & $\mathrm{TX}$ & TACE & TACE & TACE & $\mathrm{TX}$ & Sorafenib & 49.31 & 29117.65 \\
\hline 30 & TACE & $\mathrm{TX}$ & TACE & TACE & TACE & $\mathrm{TX}$ & $\mathrm{DN}$ & 49.28 & 29059.21 \\
\hline 31 & TACE & $\mathrm{TX}$ & TACE & TACE & TACE & TACE & Sorafenib & 49.31 & 29117.65 \\
\hline 32 & TACE & $\mathrm{TX}$ & TACE & TACE & TACE & TACE & DN & 49.28 & 29059.21 \\
\hline 33 & TACE & TACE & $\mathrm{TX}$ & $\mathrm{TX}$ & $\mathrm{TX}$ & $\mathrm{TX}$ & Sorafenib & 77.71 & 271092.90 \\
\hline 34 & TACE & TACE & $\mathrm{TX}$ & $\mathrm{TX}$ & $\mathrm{TX}$ & $\mathrm{TX}$ & DN & 77.67 & 271034.45 \\
\hline 35 & TACE & TACE & $\mathrm{TX}$ & $\mathrm{TX}$ & $\mathrm{TX}$ & TACE & Sorafenib & 77.71 & 271092.90 \\
\hline 36 & TACE & TACE & $\mathrm{TX}$ & $\mathrm{TX}$ & $\mathrm{TX}$ & TACE & $\mathrm{DN}$ & 77.67 & 271034.45 \\
\hline 37 & TACE & TACE & $\mathrm{TX}$ & $\mathrm{TX}$ & TACE & $\mathrm{TX}$ & Sorafenib & 69.60 & 201960.74 \\
\hline 38 & TACE & TACE & $\mathrm{TX}$ & $\mathrm{TX}$ & TACE & $\mathrm{TX}$ & DN & 69.56 & 201902.29 \\
\hline 39 & TACE & TACE & $\mathrm{TX}$ & $\mathrm{TX}$ & TACE & TACE & Sorafenib & 69.60 & 201960.74 \\
\hline 40 & TACE & TACE & $\mathrm{TX}$ & $\mathrm{TX}$ & TACE & TACE & $\mathrm{DN}$ & 69.56 & 201902.29 \\
\hline 41 & TACE & TACE & $\mathrm{TX}$ & TACE & $\mathrm{TX}$ & $\mathrm{TX}$ & Sorafenib & 65.54 & 167381.97 \\
\hline 42 & TACE & TACE & $\mathrm{TX}$ & TACE & $\mathrm{TX}$ & $\mathrm{TX}$ & DN & 65.50 & 167323.53 \\
\hline 43 & TACE & TACE & $\mathrm{TX}$ & TACE & $\mathrm{TX}$ & TACE & Sorafenib & 65.54 & 167381.97 \\
\hline 44 & TACE & TACE & $\mathrm{TX}$ & TACE & $\mathrm{TX}$ & TACE & $\mathrm{DN}$ & 65.50 & 167323.53 \\
\hline 45 & TACE & TACE & $\mathrm{TX}$ & TACE & TACE & $\mathrm{TX}$ & Sorafenib & 57.43 & 98249.81 \\
\hline 46 & TACE & TACE & $\mathrm{TX}$ & TACE & TACE & $\mathrm{TX}$ & $\mathrm{DN}$ & 57.39 & 98191.37 \\
\hline 47 & TACE & TACE & $\mathrm{TX}$ & TACE & TACE & TACE & Sorafenib & 57.43 & 98249.81 \\
\hline 48 & TACE & TACE & $\mathrm{TX}$ & TACE & TACE & TACE & $\mathrm{DN}$ & 57.39 & 98191.37 \\
\hline 49 & TACE & TACE & TACE & $\mathrm{TX}$ & $\mathrm{TX}$ & $\mathrm{TX}$ & Sorafenib & 69.60 & 201960.74 \\
\hline 50 & TACE & TACE & TACE & $\mathrm{TX}$ & $\mathrm{TX}$ & $\mathrm{TX}$ & $\mathrm{DN}$ & 69.56 & 201902.29 \\
\hline 51 & TACE & TACE & TACE & $\mathrm{TX}$ & $\mathrm{TX}$ & TACE & Sorafenib & 69.60 & 201960.74 \\
\hline 52 & TACE & TACE & TACE & $\mathrm{TX}$ & $\mathrm{TX}$ & TACE & DN & 69.56 & 201902.29 \\
\hline 53 & TACE & TACE & TACE & $\mathrm{TX}$ & TACE & $\mathrm{TX}$ & Sorafenib & 61.48 & 132828.58 \\
\hline 54 & TACE & TACE & TACE & $\mathrm{TX}$ & TACE & $\mathrm{TX}$ & $\mathrm{DN}$ & 61.45 & 132770.13 \\
\hline 55 & TACE & TACE & TACE & $\mathrm{TX}$ & TACE & TACE & Sorafenib & 61.48 & 132828.58 \\
\hline 56 & TACE & TACE & TACE & $\mathrm{TX}$ & TACE & TACE & $\mathrm{DN}$ & 61.45 & 132770.13 \\
\hline 57 & TACE & TACE & TACE & TACE & $\mathrm{TX}$ & $\mathrm{TX}$ & Sorafenib & 57.43 & 98249.81 \\
\hline 58 & TACE & TACE & TACE & TACE & $\mathrm{TX}$ & TX & DN & 57.39 & 98191.37 \\
\hline
\end{tabular}




\begin{tabular}{|c|c|c|c|c|c|c|c|c|c|}
\hline 59 & TACE & TACE & TACE & TACE & $\mathrm{TX}$ & TACE & Sorafenib & 57.43 & 98249.81 \\
\hline 60 & TACE & TACE & TACE & TACE & $\mathrm{TX}$ & TACE & $\mathrm{DN}$ & 57.39 & 98191.37 \\
\hline 61 & TACE & TACE & TACE & TACE & TACE & $\mathrm{TX}$ & Sorafenib & 49.31 & 29117.65 \\
\hline 62 & TACE & TACE & TACE & TACE & TACE & $\mathrm{TX}$ & DN & 49.28 & 29059.21 \\
\hline 63 & TACE & TACE & TACE & TACE & TACE & TACE & Sorafenib & 49.31 & 29117.65 \\
\hline 64 & TACE & TACE & TACE & TACE & TACE & TACE & $\mathrm{DN}$ & 49.28 & 29059.21 \\
\hline 65 & RFA & $\mathrm{TX}$ & $\mathrm{TX}$ & $\mathrm{TX}$ & $\mathrm{TX}$ & $\mathrm{TX}$ & Sorafenib & 77.48 & 266347.78 \\
\hline 66 & RFA & $\mathrm{TX}$ & $\mathrm{TX}$ & $\mathrm{TX}$ & $\mathrm{TX}$ & $\mathrm{TX}$ & DN & 77.46 & 266307.25 \\
\hline 67 & RFA & $\mathrm{TX}$ & $\mathrm{TX}$ & $\mathrm{TX}$ & $\mathrm{TX}$ & RFA & Sorafenib & 77.48 & 266347.78 \\
\hline 68 & RFA & $\mathrm{TX}$ & $\mathrm{TX}$ & $\mathrm{TX}$ & $\mathrm{TX}$ & RFA & DN & 77.46 & 266307.25 \\
\hline 69 & RFA & $\mathrm{TX}$ & $\mathrm{TX}$ & TX & RFA & $\mathrm{TX}$ & Sorafenib & 77.48 & 266347.78 \\
\hline 70 & RFA & $\mathrm{TX}$ & $\mathrm{TX}$ & $\mathrm{TX}$ & RFA & $\mathrm{TX}$ & DN & 77.46 & 266307.25 \\
\hline 71 & RFA & $\mathrm{TX}$ & $\mathrm{TX}$ & $\mathrm{TX}$ & RFA & RFA & Sorafenib & 77.48 & 266347.78 \\
\hline 72 & RFA & $\mathrm{TX}$ & $\mathrm{TX}$ & $\mathrm{TX}$ & RFA & RFA & DN & 77.46 & 266307.25 \\
\hline 73 & RFA & $\mathrm{TX}$ & $\mathrm{TX}$ & RFA & $\mathrm{TX}$ & $\mathrm{TX}$ & Sorafenib & 60.65 & 19714.48 \\
\hline 74 & RFA & $\mathrm{TX}$ & TX & RFA & $\mathrm{TX}$ & $\mathrm{TX}$ & DN & 60.62 & 19673.95 \\
\hline 75 & RFA & TX & $\mathrm{TX}$ & RFA & $\mathrm{TX}$ & RFA & Sorafenib & 60.65 & 19714.48 \\
\hline 76 & RFA & $\mathrm{TX}$ & $\mathrm{TX}$ & RFA & $\mathrm{TX}$ & RFA & DN & 60.62 & 19673.95 \\
\hline 77 & RFA & $\mathrm{TX}$ & $\mathrm{TX}$ & RFA & RFA & $\mathrm{TX}$ & Sorafenib & 60.65 & 19714.48 \\
\hline 78 & RFA & $\mathrm{TX}$ & $\mathrm{TX}$ & RFA & RFA & $\mathrm{TX}$ & DN & 60.62 & 19673.95 \\
\hline 79 & RFA & $\mathrm{TX}$ & $\mathrm{TX}$ & RFA & RFA & RFA & Sorafenib & 60.65 & 19714.48 \\
\hline 80 & RFA & $\mathrm{TX}$ & $\mathrm{TX}$ & RFA & RFA & RFA & DN & 60.62 & 19673.95 \\
\hline 81 & RFA & $\mathrm{TX}$ & RFA & $\mathrm{TX}$ & $\mathrm{TX}$ & $\mathrm{TX}$ & Sorafenib & 77.48 & 266347.78 \\
\hline 82 & RFA & $\mathrm{TX}$ & RFA & $\mathrm{TX}$ & $\mathrm{TX}$ & $\mathrm{TX}$ & DN & 77.46 & 266307.25 \\
\hline 83 & RFA & $\mathrm{TX}$ & RFA & $\mathrm{TX}$ & $\mathrm{TX}$ & RFA & Sorafenib & 77.48 & 266347.78 \\
\hline 84 & RFA & $\mathrm{TX}$ & RFA & $\mathrm{TX}$ & $\mathrm{TX}$ & RFA & DN & 77.46 & 266307.25 \\
\hline 85 & RFA & $\mathrm{TX}$ & RFA & $\mathrm{TX}$ & RFA & $\mathrm{TX}$ & Sorafenib & 77.48 & 266347.78 \\
\hline 86 & RFA & $\mathrm{TX}$ & RFA & $\mathrm{TX}$ & RFA & $\mathrm{TX}$ & DN & 77.46 & 266307.25 \\
\hline 87 & RFA & $\mathrm{TX}$ & RFA & $\mathrm{TX}$ & RFA & RFA & Sorafenib & 77.48 & 266347.78 \\
\hline 88 & RFA & $\mathrm{TX}$ & RFA & $\mathrm{TX}$ & RFA & RFA & $\mathrm{DN}$ & 77.46 & 266307.25 \\
\hline 89 & RFA & $\mathrm{TX}$ & RFA & RFA & $\mathrm{TX}$ & $\mathrm{TX}$ & Sorafenib & 60.65 & 19714.48 \\
\hline 90 & RFA & $\mathrm{TX}$ & RFA & RFA & $\mathrm{TX}$ & $\mathrm{TX}$ & DN & 60.62 & 19673.95 \\
\hline 91 & RFA & $\mathrm{TX}$ & RFA & RFA & $\mathrm{TX}$ & RFA & Sorafenib & 60.65 & 19714.48 \\
\hline 92 & RFA & $\mathrm{TX}$ & RFA & RFA & $\mathrm{TX}$ & RFA & DN & 60.62 & 19673.95 \\
\hline 93 & RFA & $\mathrm{TX}$ & RFA & RFA & RFA & $\mathrm{TX}$ & Sorafenib & 60.65 & 19714.48 \\
\hline 94 & RFA & $\mathrm{TX}$ & RFA & RFA & RFA & $\mathrm{TX}$ & DN & 60.62 & 19673.95 \\
\hline 95 & RFA & $\mathrm{TX}$ & RFA & RFA & RFA & RFA & Sorafenib & 60.65 & 19714.48 \\
\hline 96 & RFA & $\mathrm{TX}$ & RFA & RFA & RFA & RFA & $\mathrm{DN}$ & 60.62 & 19673.95 \\
\hline 97 & RFA & RFA & $\mathrm{TX}$ & $\mathrm{TX}$ & $\mathrm{TX}$ & $\mathrm{TX}$ & Sorafenib & 77.48 & 266347.78 \\
\hline 98 & RFA & RFA & $\mathrm{TX}$ & $\mathrm{TX}$ & $\mathrm{TX}$ & $\mathrm{TX}$ & DN & 77.46 & 266307.25 \\
\hline 99 & RFA & RFA & $\mathrm{TX}$ & $\mathrm{TX}$ & $\mathrm{TX}$ & RFA & Sorafenib & 77.48 & 266347.78 \\
\hline 100 & RFA & RFA & $\mathrm{TX}$ & $\mathrm{TX}$ & $\mathrm{TX}$ & RFA & DN & 77.46 & 266307.25 \\
\hline 101 & RFA & RFA & $\mathrm{TX}$ & $\mathrm{TX}$ & RFA & $\mathrm{TX}$ & Sorafenib & 77.48 & 266347.78 \\
\hline 102 & RFA & RFA & $\mathrm{TX}$ & $\mathrm{TX}$ & RFA & $\mathrm{TX}$ & DN & 77.46 & 266307.25 \\
\hline 103 & RFA & RFA & $\mathrm{TX}$ & $\mathrm{TX}$ & RFA & RFA & Sorafenib & 77.48 & 266347.78 \\
\hline 104 & RFA & RFA & $\mathrm{TX}$ & TX & RFA & RFA & DN & 77.46 & 266307.25 \\
\hline
\end{tabular}




\begin{tabular}{|c|c|c|c|c|c|c|c|c|c|c|}
\hline 105 & RFA & RFA & $\mathrm{TX}$ & RFA & $\mathrm{TX}$ & $\mathrm{TX}$ & Sorafenib & 60.65 & 19714.48 & \\
\hline 106 & RFA & RFA & $\mathrm{TX}$ & RFA & $\mathrm{TX}$ & $\mathrm{TX}$ & DN & 60.62 & 19673.95 & \\
\hline 107 & RFA & RFA & $\mathrm{TX}$ & RFA & $\mathrm{TX}$ & RFA & Sorafenib & 60.65 & 19714.48 & \\
\hline 108 & RFA & RFA & $\mathrm{TX}$ & RFA & $\mathrm{TX}$ & RFA & DN & 60.62 & 19673.95 & \\
\hline 109 & RFA & RFA & $\mathrm{TX}$ & RFA & RFA & $\mathrm{TX}$ & Sorafenib & 60.65 & 19714.48 & \\
\hline 110 & RFA & RFA & $\mathrm{TX}$ & RFA & RFA & $\mathrm{TX}$ & DN & 60.62 & 19673.95 & \\
\hline 111 & RFA & RFA & $\mathrm{TX}$ & RFA & RFA & RFA & Sorafenib & 60.65 & 19714.48 & \\
\hline 112 & RFA & RFA & $\mathrm{TX}$ & RFA & RFA & RFA & DN & 60.62 & 19673.95 & \\
\hline 113 & RFA & RFA & RFA & $\mathrm{TX}$ & $\mathrm{TX}$ & $\mathrm{TX}$ & Sorafenib & 77.48 & 266347.78 & \\
\hline 114 & RFA & RFA & RFA & $\mathrm{TX}$ & $\mathrm{TX}$ & $\mathrm{TX}$ & DN & 77.46 & 266307.25 & \\
\hline 115 & RFA & RFA & RFA & $\mathrm{TX}$ & $\mathrm{TX}$ & RFA & Sorafenib & 77.48 & 266347.78 & \\
\hline 116 & RFA & RFA & RFA & $\mathrm{TX}$ & $\mathrm{TX}$ & RFA & DN & 77.46 & 266307.25 & \\
\hline 117 & RFA & RFA & RFA & $\mathrm{TX}$ & RFA & $\mathrm{TX}$ & Sorafenib & 77.48 & 266347.78 & \\
\hline 118 & RFA & RFA & RFA & $\mathrm{TX}$ & RFA & $\mathrm{TX}$ & DN & 77.46 & 266307.25 & \\
\hline 119 & RFA & RFA & RFA & $\mathrm{TX}$ & RFA & RFA & Sorafenib & 77.48 & 266347.78 & \\
\hline 120 & RFA & RFA & RFA & $\mathrm{TX}$ & RFA & RFA & DN & 77.46 & 266307.25 & \\
\hline 121 & RFA & RFA & RFA & RFA & $\mathrm{TX}$ & $\mathrm{TX}$ & Sorafenib & 60.65 & 19714.48 & \\
\hline 122 & RFA & RFA & RFA & RFA & $\mathrm{TX}$ & $\mathrm{TX}$ & DN & 60.62 & 19673.95 & \\
\hline 123 & RFA & RFA & RFA & RFA & $\mathrm{TX}$ & RFA & Sorafenib & 60.65 & 19714.48 & \\
\hline 124 & RFA & RFA & RFA & RFA & $\mathrm{TX}$ & RFA & $\mathrm{DN}$ & 60.62 & 19673.95 & \\
\hline 125 & RFA & RFA & RFA & RFA & RFA & $\mathrm{TX}$ & Sorafenib & 60.65 & 19714.48 & \\
\hline 126 & RFA & RFA & RFA & RFA & RFA & $\mathrm{TX}$ & DN & 60.62 & 19673.95 & \\
\hline 127 & RFA & RFA & RFA & RFA & RFA & RFA & Sorafenib & 60.65 & 19714.48 & \\
\hline 128 & RFA & RFA & RFA & RFA & RFA & RFA & DN & 60.62 & 19673.95 & \\
\hline 129 & $\mathrm{DN}$ & $\mathrm{TX}$ & $\mathrm{TX}$ & $\mathrm{TX}$ & $\mathrm{TX}$ & $\mathrm{TX}$ & Sorafenib & 76.24 & 186343.65 & \\
\hline 130 & DN & $\mathrm{TX}$ & $\mathrm{TX}$ & $\mathrm{TX}$ & $\mathrm{TX}$ & $\mathrm{TX}$ & DN & 76.21 & 186310.12 & \\
\hline 131 & DN & $\mathrm{TX}$ & $\mathrm{TX}$ & $\mathrm{TX}$ & $\mathrm{TX}$ & $\mathrm{DN}$ & Sorafenib & 53.82 & 185198.11 & $\mathrm{X}$ \\
\hline 132 & DN & $\mathrm{TX}$ & $\mathrm{TX}$ & $\mathrm{TX}$ & $\mathrm{TX}$ & $\mathrm{DN}$ & DN & 53.80 & 185164.58 & $\mathrm{X}$ \\
\hline 133 & DN & $\mathrm{TX}$ & $\mathrm{TX}$ & $\mathrm{TX}$ & $\mathrm{DN}$ & $\mathrm{TX}$ & Sorafenib & 53.82 & 109659.01 & $\mathrm{X}$ \\
\hline 134 & DN & $\mathrm{TX}$ & $\mathrm{TX}$ & $\mathrm{TX}$ & $\mathrm{DN}$ & $\mathrm{TX}$ & DN & 53.80 & 109625.48 & $\mathrm{X}$ \\
\hline 135 & DN & $\mathrm{TX}$ & $\mathrm{TX}$ & $\mathrm{TX}$ & $\mathrm{DN}$ & $\mathrm{DN}$ & Sorafenib & 31.41 & 108513.47 & $\mathrm{X}$ \\
\hline 136 & DN & $\mathrm{TX}$ & $\mathrm{TX}$ & $\mathrm{TX}$ & $\mathrm{DN}$ & $\mathrm{DN}$ & DN & 31.39 & 108479.94 & $\mathrm{X}$ \\
\hline 137 & DN & $\mathrm{TX}$ & $\mathrm{TX}$ & $\mathrm{DN}$ & $\mathrm{TX}$ & $\mathrm{TX}$ & Sorafenib & 58.30 & 124984.85 & $\mathrm{X}$ \\
\hline 138 & DN & $\mathrm{TX}$ & $\mathrm{TX}$ & $\mathrm{DN}$ & $\mathrm{TX}$ & $\mathrm{TX}$ & DN & 58.28 & 124951.32 & $\mathrm{X}$ \\
\hline 139 & $\mathrm{DN}$ & $\mathrm{TX}$ & $\mathrm{TX}$ & $\mathrm{DN}$ & $\mathrm{TX}$ & $\mathrm{DN}$ & Sorafenib & 35.89 & 123839.32 & $\mathrm{X}$ \\
\hline 140 & DN & $\mathrm{TX}$ & $\mathrm{TX}$ & $\mathrm{DN}$ & $\mathrm{TX}$ & $\mathrm{DN}$ & DN & 35.87 & 123805.78 & $\mathrm{X}$ \\
\hline 141 & DN & $\mathrm{TX}$ & $\mathrm{TX}$ & $\mathrm{DN}$ & $\mathrm{DN}$ & $\mathrm{TX}$ & Sorafenib & 35.89 & 48300.22 & $\mathrm{X}$ \\
\hline 142 & DN & $\mathrm{TX}$ & $\mathrm{TX}$ & $\mathrm{DN}$ & $\mathrm{DN}$ & $\mathrm{TX}$ & DN & 35.87 & 48266.68 & $\mathrm{X}$ \\
\hline 143 & $\mathrm{DN}$ & $\mathrm{TX}$ & $\mathrm{TX}$ & $\mathrm{DN}$ & $\mathrm{DN}$ & $\mathrm{DN}$ & Sorafenib & 13.48 & 47154.68 & $\mathrm{X}$ \\
\hline 144 & DN & $\mathrm{TX}$ & $\mathrm{TX}$ & $\mathrm{DN}$ & $\mathrm{DN}$ & $\mathrm{DN}$ & DN & 13.45 & 47121.14 & $\mathrm{X}$ \\
\hline 145 & DN & $\mathrm{TX}$ & $\mathrm{DN}$ & $\mathrm{TX}$ & $\mathrm{TX}$ & $\mathrm{TX}$ & Sorafenib & 62.78 & 140310.70 & \\
\hline 146 & $\mathrm{DN}$ & $\mathrm{TX}$ & $\mathrm{DN}$ & $\mathrm{TX}$ & $\mathrm{TX}$ & $\mathrm{TX}$ & DN & 62.76 & 140277.16 & \\
\hline 147 & DN & $\mathrm{TX}$ & $\mathrm{DN}$ & $\mathrm{TX}$ & $\mathrm{TX}$ & $\mathrm{DN}$ & Sorafenib & 40.37 & 139165.16 & $X$ \\
\hline 148 & DN & $\mathrm{TX}$ & $\mathrm{DN}$ & $\mathrm{TX}$ & $\mathrm{TX}$ & $\mathrm{DN}$ & DN & 40.35 & 139131.62 & $\mathrm{X}$ \\
\hline 149 & $\mathrm{DN}$ & $\mathrm{TX}$ & $\mathrm{DN}$ & $\mathrm{TX}$ & $\mathrm{DN}$ & $\mathrm{TX}$ & Sorafenib & 40.37 & 63626.06 & $\mathrm{X}$ \\
\hline 150 & $\mathrm{DN}$ & $\mathrm{TX}$ & $\mathrm{DN}$ & $\mathrm{TX}$ & $\mathrm{DN}$ & $\mathrm{TX}$ & $\mathrm{DN}$ & 40.35 & 63592.52 & $\mathrm{X}$ \\
\hline
\end{tabular}




\begin{tabular}{|c|c|c|c|c|c|c|c|c|c|}
\hline 151 & $\mathrm{DN}$ & $\mathrm{TX}$ & $\mathrm{DN}$ & $\mathrm{TX}$ & $\mathrm{DN}$ & $\mathrm{DN}$ & Sorafenib & 17.96 & 62480.52 \\
\hline 152 & DN & $\mathrm{TX}$ & $\mathrm{DN}$ & $\mathrm{TX}$ & $\mathrm{DN}$ & $\mathrm{DN}$ & DN & 17.93 & 62446.99 \\
\hline 153 & DN & $\mathrm{TX}$ & $\mathrm{DN}$ & DN & $\mathrm{TX}$ & $\mathrm{TX}$ & Sorafenib & 44.85 & 78951.90 \\
\hline 154 & DN & $\mathrm{TX}$ & $\mathrm{DN}$ & DN & $\mathrm{TX}$ & $\mathrm{TX}$ & DN & 44.83 & 78918.37 \\
\hline 155 & DN & $\mathrm{TX}$ & $\mathrm{DN}$ & DN & $\mathrm{TX}$ & $\mathrm{DN}$ & Sorafenib & 22.44 & 77806.36 \\
\hline 156 & DN & $\mathrm{TX}$ & $\mathrm{DN}$ & DN & $\mathrm{TX}$ & $\mathrm{DN}$ & DN & 22.41 & 77772.83 \\
\hline 157 & DN & $\mathrm{TX}$ & $\mathrm{DN}$ & DN & $\mathrm{DN}$ & $\mathrm{TX}$ & Sorafenib & 22.44 & 2267.26 \\
\hline 158 & DN & $\mathrm{TX}$ & $\mathrm{DN}$ & DN & $\mathrm{DN}$ & $\mathrm{TX}$ & DN & 22.41 & 2233.73 \\
\hline 159 & DN & $\mathrm{TX}$ & $\mathrm{DN}$ & DN & $\mathrm{DN}$ & $\mathrm{DN}$ & Sorafenib & 0.02 & 1121.72 \\
\hline 160 & DN & $\mathrm{TX}$ & $\mathrm{DN}$ & DN & $\mathrm{DN}$ & $\mathrm{DN}$ & $\mathrm{DN}$ & 0.00 & 1088.19 \\
\hline 161 & DN & $\mathrm{DN}$ & $\mathrm{TX}$ & $\mathrm{TX}$ & $\mathrm{TX}$ & $\mathrm{TX}$ & Sorafenib & 76.24 & 186343.65 \\
\hline 162 & DN & $\mathrm{DN}$ & $\mathrm{TX}$ & $\mathrm{TX}$ & $\mathrm{TX}$ & $\mathrm{TX}$ & DN & 76.21 & 186310.12 \\
\hline 163 & $\mathrm{DN}$ & $\mathrm{DN}$ & $\mathrm{TX}$ & $\mathrm{TX}$ & $\mathrm{TX}$ & $\mathrm{DN}$ & Sorafenib & 53.82 & 185198.11 \\
\hline 164 & DN & $\mathrm{DN}$ & $\mathrm{TX}$ & $\mathrm{TX}$ & $\mathrm{TX}$ & $\mathrm{DN}$ & DN & 53.80 & 185164.58 \\
\hline 165 & DN & $\mathrm{DN}$ & $\mathrm{TX}$ & $\mathrm{TX}$ & $\mathrm{DN}$ & $\mathrm{TX}$ & Sorafenib & 53.82 & 109659.01 \\
\hline 166 & DN & $\mathrm{DN}$ & $\mathrm{TX}$ & $\mathrm{TX}$ & $\mathrm{DN}$ & $\mathrm{TX}$ & DN & 53.80 & 109625.48 \\
\hline 167 & DN & $\mathrm{DN}$ & $\mathrm{TX}$ & $\mathrm{TX}$ & $\mathrm{DN}$ & $\mathrm{DN}$ & Sorafenib & 31.41 & 108513.47 \\
\hline 168 & DN & $\mathrm{DN}$ & $\mathrm{TX}$ & $\mathrm{TX}$ & $\mathrm{DN}$ & $\mathrm{DN}$ & $\mathrm{DN}$ & 31.39 & 108479.94 \\
\hline 169 & DN & $\mathrm{DN}$ & $\mathrm{TX}$ & DN & $\mathrm{TX}$ & $\mathrm{TX}$ & Sorafenib & 58.30 & 124984.85 \\
\hline 170 & DN & $\mathrm{DN}$ & $\mathrm{TX}$ & DN & $\mathrm{TX}$ & $\mathrm{TX}$ & DN & 58.28 & 124951.32 \\
\hline 171 & DN & $\mathrm{DN}$ & $\mathrm{TX}$ & DN & $\mathrm{TX}$ & $\mathrm{DN}$ & Sorafenib & 35.89 & 123839.32 \\
\hline 172 & DN & $\mathrm{DN}$ & $\mathrm{TX}$ & DN & $\mathrm{TX}$ & $\mathrm{DN}$ & DN & 35.87 & 123805.78 \\
\hline 173 & DN & $\mathrm{DN}$ & $\mathrm{TX}$ & DN & $\mathrm{DN}$ & $\mathrm{TX}$ & Sorafenib & 35.89 & 48300.22 \\
\hline 174 & DN & $\mathrm{DN}$ & $\mathrm{TX}$ & DN & $\mathrm{DN}$ & $\mathrm{TX}$ & DN & 35.87 & 48266.68 \\
\hline 175 & DN & $\mathrm{DN}$ & $\mathrm{TX}$ & DN & $\mathrm{DN}$ & $\mathrm{DN}$ & Sorafenib & 13.48 & 47154.68 \\
\hline 176 & DN & $\mathrm{DN}$ & $\mathrm{TX}$ & DN & $\mathrm{DN}$ & DN & DN & 13.45 & 47121.14 \\
\hline 177 & DN & $\mathrm{DN}$ & $\mathrm{DN}$ & $\mathrm{TX}$ & $\mathrm{TX}$ & $\mathrm{TX}$ & Sorafenib & 62.78 & 140310.70 \\
\hline 178 & DN & $\mathrm{DN}$ & $\mathrm{DN}$ & $\mathrm{TX}$ & $\mathrm{TX}$ & $\mathrm{TX}$ & DN & 62.76 & 140277.16 \\
\hline 179 & DN & $\mathrm{DN}$ & $\mathrm{DN}$ & $\mathrm{TX}$ & $\mathrm{TX}$ & $\mathrm{DN}$ & Sorafenib & 40.37 & 139165.16 \\
\hline 180 & DN & $\mathrm{DN}$ & $\mathrm{DN}$ & $\mathrm{TX}$ & $\mathrm{TX}$ & $\mathrm{DN}$ & DN & 40.35 & 139131.62 \\
\hline 181 & DN & $\mathrm{DN}$ & $\mathrm{DN}$ & $\mathrm{TX}$ & $\mathrm{DN}$ & $\mathrm{TX}$ & Sorafenib & 40.37 & 63626.06 \\
\hline 182 & DN & $\mathrm{DN}$ & $\mathrm{DN}$ & $\mathrm{TX}$ & $\mathrm{DN}$ & $\mathrm{TX}$ & DN & 40.35 & 63592.52 \\
\hline 183 & DN & $\mathrm{DN}$ & $\mathrm{DN}$ & $\mathrm{TX}$ & $\mathrm{DN}$ & $\mathrm{DN}$ & Sorafenib & 17.96 & 62480.52 \\
\hline 184 & DN & $\mathrm{DN}$ & $\mathrm{DN}$ & $\mathrm{TX}$ & $\mathrm{DN}$ & $\mathrm{DN}$ & DN & 17.93 & 62446.99 \\
\hline 185 & DN & $\mathrm{DN}$ & $\mathrm{DN}$ & DN & $\mathrm{TX}$ & $\mathrm{TX}$ & Sorafenib & 44.85 & 78951.90 \\
\hline 186 & DN & $\mathrm{DN}$ & $\mathrm{DN}$ & DN & $\mathrm{TX}$ & $\mathrm{TX}$ & DN & 44.83 & 78918.37 \\
\hline 187 & DN & $\mathrm{DN}$ & DN & DN & $\mathrm{TX}$ & $\mathrm{DN}$ & Sorafenib & 22.44 & 77806.36 \\
\hline 188 & DN & $\mathrm{DN}$ & $\mathrm{DN}$ & DN & $\mathrm{TX}$ & $\mathrm{DN}$ & DN & 22.41 & 77772.83 \\
\hline 189 & DN & $\mathrm{DN}$ & $\mathrm{DN}$ & DN & $\mathrm{DN}$ & $\mathrm{TX}$ & Sorafenib & 22.44 & 2267.26 \\
\hline 190 & DN & $\mathrm{DN}$ & $\mathrm{DN}$ & DN & $\mathrm{DN}$ & $\mathrm{TX}$ & DN & 22.41 & 2233.73 \\
\hline 191 & DN & $\mathrm{DN}$ & DN & DN & $\mathrm{DN}$ & $\mathrm{DN}$ & Sorafenib & 0.02 & 1121.72 \\
\hline 192 & DN & $\mathrm{DN}$ & $\mathrm{DN}$ & DN & $\mathrm{DN}$ & $\mathrm{DN}$ & DN & 0.00 & 1088.19 \\
\hline
\end{tabular}




\section{APPENDIX E: GRAPHS OF STRATEGIES (ALL AND OPTIMAL)}
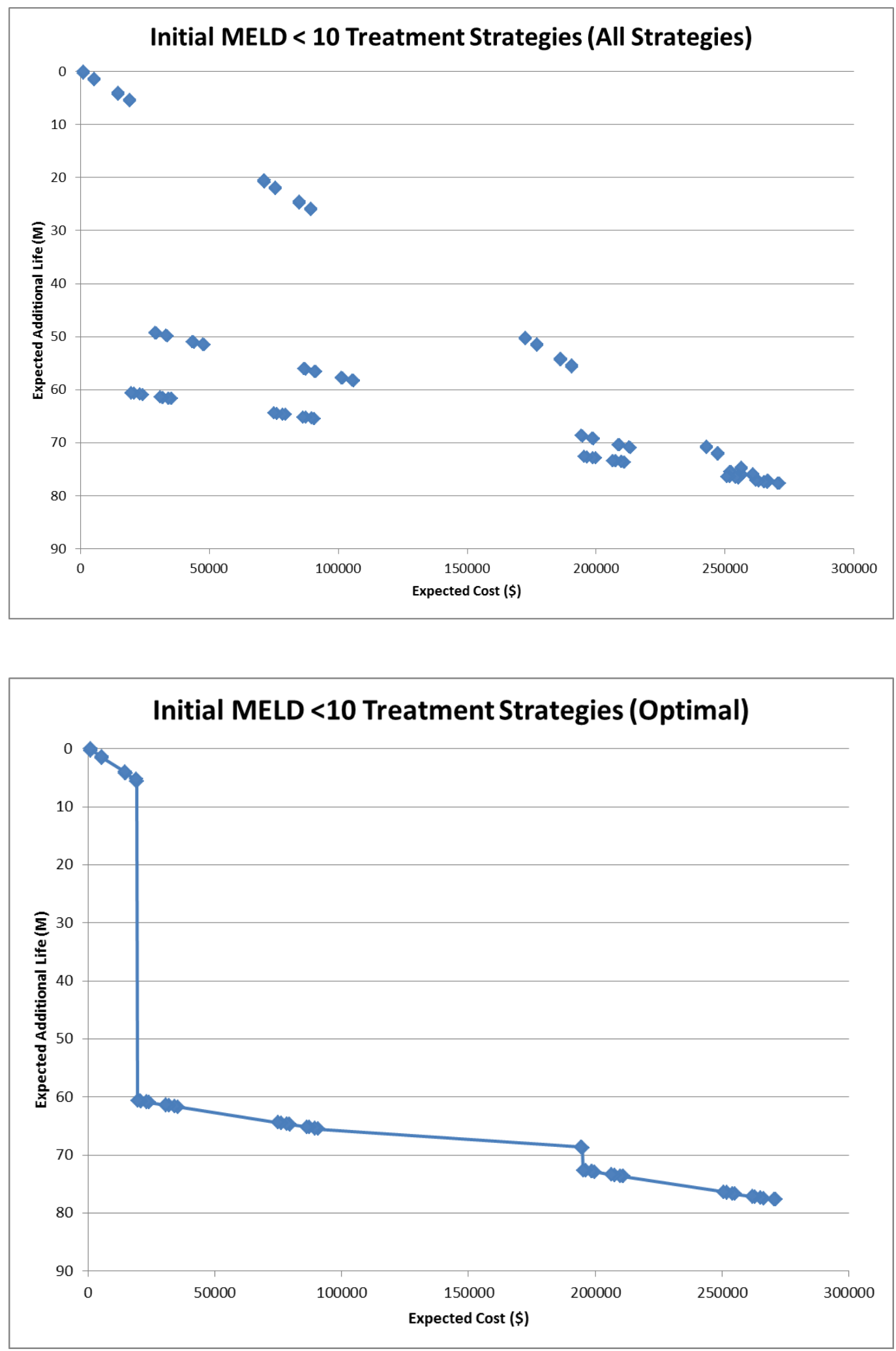

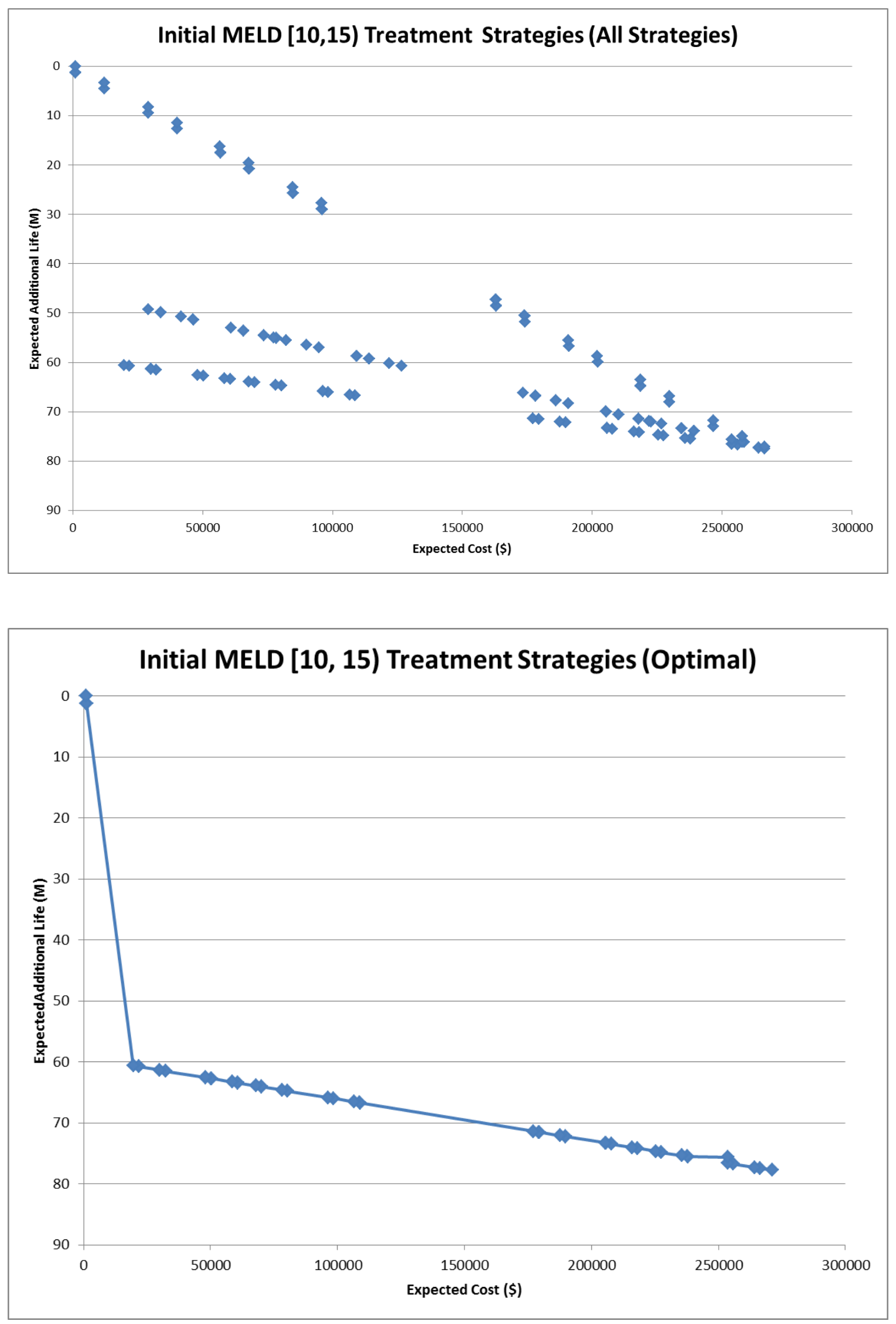

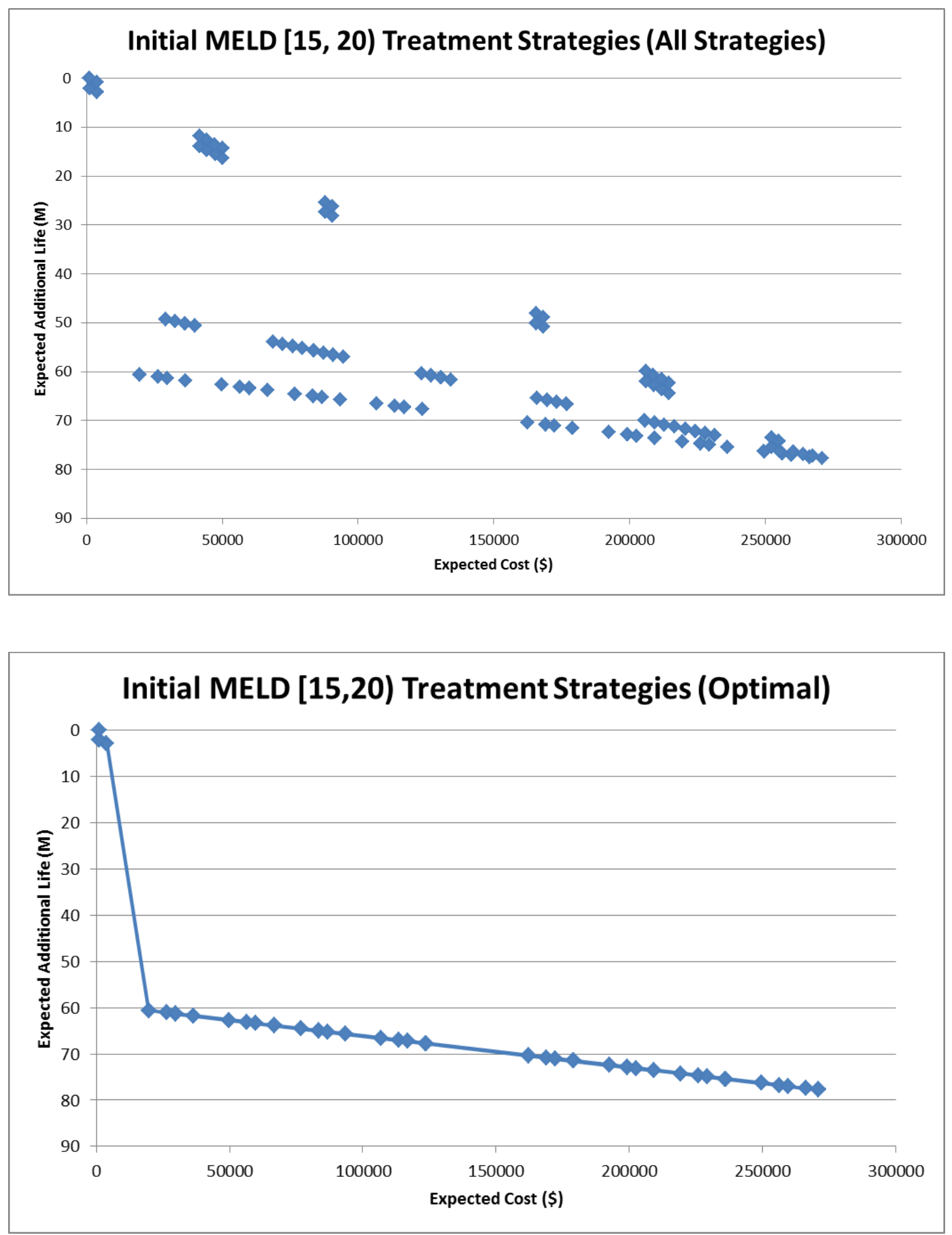

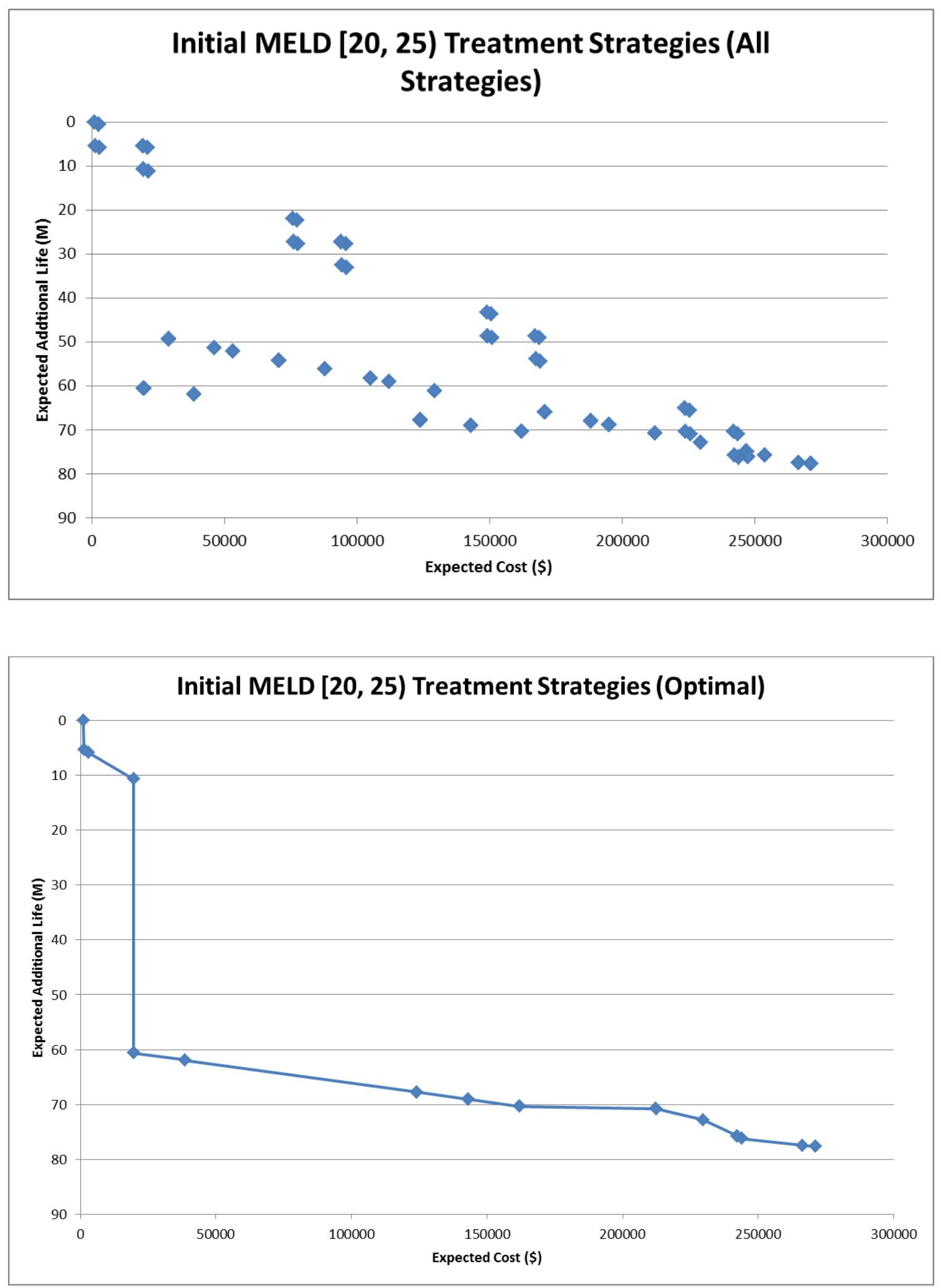

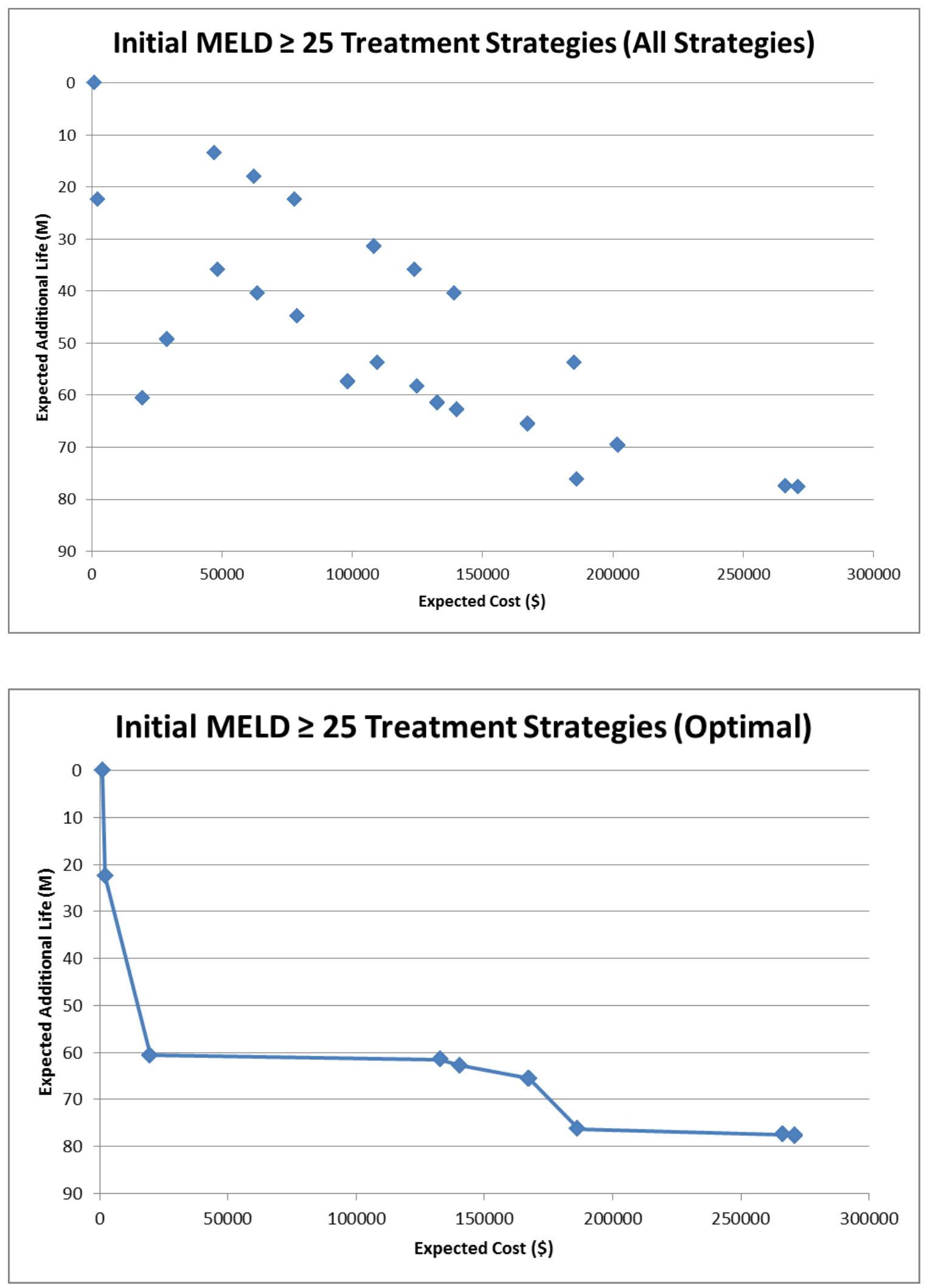